\author{
Universidade de São Paulo \\ Instituto de Física
}

\title{
Descoerência em Oscilações de Neutrinos no Experimento IceCube
}

\author{
Gustavo Figueiredo Severiano Alves
}

Orientadora: Profa. Dra. Renata Zukanovich Funchal.

Banca Examinadora:

Profa. Dra. Renata Zukanovich Funchal (IF/USP)

Prof. Dr. Hiroshi Nunokawa (PUC/RJ)

Prof. Dr. Orlando Luis Goulart Peres (IFGW/UNICAMP) 
FICHA CATALOGRÁFICA

Preparada pelo Serviço de Biblioteca e Informação do Instituto de Física da Universidade de São Paulo

Alves, Gustavo Figueiredo Severiano

Descoerência em oscilações de neutrinos no Experimento IceCube. São Paulo, 2020.

Dissertação (Mestrado) - Universidade de São Paulo. Instituto de Física. Depto. de Física Matemática.

Orientador: Profa. Dra. Renata Zukanovich Funchal

Área de Concentração: Física de partículas elementares e campos

Unitermos: 1. Física de neutrinos; 2. Sistemas quânticos abertos; 3. Oscilação de neutrinos; 4. IceCube. 


\title{
University of São Paulo \\ Physics Institute
}

\section{Decoherence in Neutrino Oscillations in the IceCube Experiment}

\author{
Gustavo Figueiredo Severiano Alves
}

Supervisor: Prof. Dr. Renata Zukanovich Funchal.

Dissertation submitted to the Physics Institute of the University of São Paulo in partial fulfillment of the requirements for the degree of Master of Science.

Examining Committee:

Prof. Dr. Renata Zukanovich Funchal (IF/USP)

Prof. Dr. Hiroshi Nunokawa (PUC/RJ)

Prof. Dr. Orlando Luis Goulart Peres (IFGW/UNICAMP) 



\section{ACKNOWLEDGMENTS - AGRADECIMENTOS}

$\mathrm{D}$

ESDE o dia em que deixei Minas Gerais para iniciar meus estudos em São Paulo, minha vida mudou drasticamente. O término desse trabalho marca seis anos que decidi estudar Física mais a fundo com o inocente propósito de entender melhor os porquês do mundo. A trajetória não foi fácil, porém, tem me retribuído muito mais do que os sacrifícios de enfrentá-la, não me arrependo de forma alguma da escolha que fiz em 2014.

Sempre tive laços muito fortes com meus familiares, o preço da distância é um dos mais altos que pago até hoje, portanto gostaria de agradecer, primeiramente, aos meus pais, Neusa e José Ronaldo, pela confiança em me deixar seguir um caminho que eles não conhecem, por sempre terem me alertado para a importância dos estudos, por todo amor e preocupação, por todas as advertências e claro pelo apoio financeiro que me permitiu concentrar durante esse tempo somente nos estudos, aprendi e continuo aprendendo muito com o exemplo de vocês. Agradeço também aos meus irmãos, Rafael, Maria Laura, Maria Eduarda, Felipe e Maria Eugênia, vocês são muito especiais na minha vida e sou extremamente grato por tudo que fizeram e fazem por mim, por todo apoio emocional e ajuda. Dedico esse trabalho à todos vocês, principalmente aos meus irmãos, que vejam nessa conquista inspiração para continuarem firmes no caminho que desejarem seguir. Estou sempre com vocês.

Agradeço também à minha namorada, Kauara, por ser essa pessoa incrível, seu apoio e carinho foram combustível nos dias que eu estava bem e conforto nos dias de desespero. Muito obrigado por tudo que fez e faz por mim.

Não poderia esquecer de agradecer a minha madrinha Andrea por ter me hospedado durante os meses iniciais de graduação e por sempre estar disponível para me ajudar em qualquer situação.

Agradeço aos professores que me apoiaram no ensino médio quando decidi seguir a física como profissão. Sou eternamente grato também ao professor, Sérgio Ricardo Muniz, que foi a primeira pessoa formada em física que tive contato. Olhando em retrospecto admiro muito a postura que adotou frente as minhas perguntas por email, eu era apenas um estranho com as questões mais peculiares e desprovidas de sentido e você mesmo assim não só teve muita paciência, como também respondeu todas em detalhe e me abriu portas para descobrir um mundo novo, sem ele eu provavelmente não estaria aqui hoje. Levarei seu exemplo para sempre, cada ano que passo na física vejo o quão raro pessoas como você são e como tive sorte de ter te 
conhecido.

Agradeço à todos meus amigos, em especial ao William, com quem compartilhei a maioria dos dias bons e ruins da graduação, ao Luighi pelas discussões sobre física, ajuda nas monitorias e na pesquisa e à Laura, pela amizade e apoio de longa data.

Agradeço ao pessoal da Academia Gracie Butantã, em especial Jorge, Felipe, Erik, Bruno, Pablo, Weslen e meus professores Saga e Thiago, com certeza meus dias em São Paulo foram muito mais suportáveis e felizes desde que conheci vocês.

Agradeço imensamente à minha orientadora, Renata Funchal, após escolher a Física, sua orientação foi a segunda escolha mais feliz que fiz. Sou eternamente grato por você ter aceito e por ter me dado essa grande oportunidade. Com suas explicações você me ensinou muito sobre a ciência, com suas ações você me ensinou pelo exemplo como é ser um pesquisador íntegro e a valorizar o esforço, sabendo sempre que o dia de hoje foi fácil perto do que farei amanhã. Meus limites foram gradualmente redefinidos desde que te conheci, você sempre me incentiva a fazer um pouco mais, espero ter cumprido os combinados que conversamos em sua sala dois anos atrás, muito obrigado.

I would like to thank Boris Panes for the collaboration during these years, without him this thesis would never have been finished. His ideas, questions and advices taught me how to properly do research and have guided me throughout the progress of this work, helping me to improve my understanding of physics.

Agradeço à todos funcionários do Departamento de Física Matemática por terem me ajudado com todas incumbências burocráticas e organizacionais.

O presente trabalho foi realizado com apoio do CNPq, Conselho Nacional de Desenvolvimento Científico e Tecnológico - Brasil. Agradeço pelo auxílio financeiro durante esses dois anos. 


\section{ABSTRACT}

7 HE discovery of neutrino oscillations established the fact that they are massive particles. This is a clear signal of physics beyond the Standard Model, since this theoretical framework cannot explain how the neutrino mass terms can be generated. Furthermore, because this phenomenon is strictly a quantum one it can be used to study even more the quantum realm. In this work we shall take advantage of the sensitivity of this phenomenon to new effects. Since the neutrino physics has entered a precision measurement era, we expect that such modifications can be further constrained, or even confirmed, with new experimental results. We will focus on a very special kind of effects, the Open Quantum System effects. This is an interesting theoretical framework because it is a predictive description which can also handle our lack of information about the neutrino system. For that purpose we will study the theoretical basis of neutrino oscillations and Open Quantum System effects, discuss how to include both in the time evolution of the neutrino system and investigate how the new effects can change the oscillation pattern. Finally, we will use the public data released by the IceCube experiment to analyse the consequences and also constrain such modifications.

Keywords: Neutrino Physics; Open Quantum Systems; Neutrino Oscillations; IceCube. 



\title{
RESUMO
}

\begin{abstract}
A DescoberTa do fenômeno de oscilação de neutrinos estabeleceu o fato de que eles são partículas massivas. Isso leva a sinais claros de física nova, já que no Modelo 1 Padrão não existe um mecanismo para gerar massa para essas partículas. Além disso, esse fenômeno é de natureza quântica e pode ser usado para estudar ainda em mais profundidade o mundo quântico. Nesse trabalho iremos utilizar da sensibilidade do fenômeno de oscilação para estudar efeitos novos que podem afetar o sistema de neutrinos. Como a física de neutrinos entrou na era de medidas de precisão, esperamos que as possíveis modificações associadas à esses efeitos possam ser detectadas ou severamente restringidas por novos resultados experimentais. Nesse trabalho escolhemos estudar os efeitos de Sistemas Quânticos Abertos, pois é uma descrição teórica preditiva que consegue parametrizar nossa ignorância a respeito do sistema de neutrinos. Para esse propósito, estudaremos a base téorica do fenômeno de oscilações e Sistemas Quânticos Abertos, discutiremos como incluir ambos na evolução temporal do sistema de neutrinos e investigaremos como esses efeitos novos podem alterar o padrão de oscilação de neutrinos. Após isso, vamos utilizar os dados públicos liberados pelo experimento IceCube para analisar as consequências e também colocar novos limites nessas possíveis modificações.
\end{abstract}

Palavras Chave: Física de neutrinos; Sistemas quânticos abertos; Oscilação de neutrinos; IceCube. 



\section{LIST OF FIGURES}

1.1 Feynman diagram representing the process. . . . . . . . . . . . . . 27

1.2 Representation of the processes happening at the production and detection regions. 30

2.1 The behaviour of $\operatorname{Tr}\left\{\rho^{2}\right\}$ considering the example $(2.4) \ldots \ldots$. . . . . . . 40

2.2 A necessary and sufficient condition for a map to be universal is that the initial state of the whole system must be written as a product state. On the other hand, the evolved state, in general, cannot be written in this form. Therefore, there is no guarantee that the inherited map will be a Universal Dynamical Map. Figure taken from [76]. . . . . . . . . . . . . . . . . . . . . . . . . 44 44

2.3 Summary of the evolution of the reduced state $\rho_{S} \ldots \ldots \ldots \ldots 4$

3.1 The Checker map, a useful tool to follow how an element of the Kossakowski matrix affects the Dissipator. On the left (the $3 \times 3$ matrix) is the Kossakowski matrix with only $a_{11}$ different from zero, on the right is the Dissipator corresponding to this Kossakowski matrix. . . . . . . . . . . . . . 61

3.2 $\nu_{\mu}$ survival probability as a function of the energy for the parameters described in tables 3.2 and 3.3 for each case of table 3.1 . . . . . . . . . . . . . 63

3.3 Linear entropy for the $\nu_{\mu}$ state considering the parameters from tables 3.2 and 3.3. Since in the standard scenario the state remains pure for all the energies the linear entropy is zero, therefore this case is not shown in the plot. . . . . . . . 63

3.4 Same as figure 3.2, but considering different values of the Majorana phase $\alpha$. . 64

3.5 Same as figure 3.3, but considering different values of the Majorana phase $\alpha . \quad$. 65

3.6 Same as figure 3.2, but considering different values of the Majorana phase $\alpha$. The smaller plot shows the amount of CP (CPT) violation due to Open System effects (see equation (3.28)). . . . . . . . . . . . . . . .

3.7 The $\nu_{\mu} \rightarrow \nu_{\mu}$ oscillation channel considering different values for the $\delta$ phase and setting the two Majorana phases to zero. The parameters used are summarized in tables 3.4, 3.5 and 3.6. The Opn, Std and Mat abbreviations stand for open, standard and matter, respectively. All the Open System cases were obtained by considering the Kossakowski matrix $a$ in equation (3.39) . . . . . . . . . . 
3.8 Same as figure 3.7, but considering the $\nu_{\mu} \rightarrow \nu_{e}$ oscillation channel. . . . . . 73

3.9 Same as figure 3.7, but considering the $\nu_{\mu} \rightarrow \nu_{\tau}$ oscillation channel. . . . . . 73

3.10 The $\nu_{\mu} \rightarrow \nu_{\mu}$ oscillation channel keeping fixed the value of the $\delta$ phase and varying $\phi_{1}$ and $\phi_{2}$. The parameters used are summarized in tables 3.4, 3.5 and 3.6. The Opn, Std and Mat abbreviations stand for open, standard and matter, respectively. Only the Open system case where $\phi_{1}$ is different from zero was obtained by considering the Kossakowski matrix $\tilde{a}$ in (3.39). . . . . . . . . . . 74

3.11 Same as figure 3.10, but considering the $\nu_{\mu} \rightarrow \nu_{e}$ oscillation channel. . . . . . 74

3.12 Same as figure 3.10, but considering the $\nu_{\mu} \rightarrow \nu_{\tau}$ oscillation channel. $\ldots . .75$

3.13 The CPT violation in the $\nu_{\mu} \rightarrow \nu_{\mu}$ channel (only CP for the other $\nu_{\mu} \rightarrow \nu_{e}$ and $\nu_{\mu} \rightarrow \nu_{\tau}$ oscillation channels) for different combination of phases. We also show a standard scenario where the $\mathrm{CPT}(\mathrm{CP})$ violation is purely due to the matter effects. The parameters used are summarized in tables 3.4, 3.5 and 3.6. The Opn, Std and Mat abbreviations stand for open, standard and matter, respectively. Only the Open system case where $\phi_{1}$ is different from zero was obtained by considering the Kossakowski matrix $\tilde{a}$ in (3.39) . . . . . . . . . 75

3.14 Same as figure 3.13, but considering the $\nu_{\mu} \rightarrow \nu_{e}$ channel. . . . . . . . . 76

3.15 Same as figure 3.13, but considering the $\nu_{\mu} \rightarrow \nu_{\tau}$ channel. . . . . . . . 76

3.16 Linear entropy for all three neutrino flavor considering only the phase $\delta$ fixed to the value $3 \pi / 2$. The Open System effects were included by using the Kossakowski matrix $a$ in equation (3.39). . . . . . . . . . . . . . . .

3.17 Linear entropy for all three neutrino flavor considering both the phases $\delta$ and $\phi_{2}$ fixed to values $3 \pi / 2$ and $\pi / 4$, respectively. The Open System effects were included by using the Kossakowski matrix $a$ in equation (3.39) . . . . . . . .

3.18 Consequences of the Open System interactions using the Kossakoski matrix $a$ in equation (3.39) for the energy range in the reach of the $\mathrm{NO} \nu \mathrm{A}$ experiment. The baseline distance is $L_{\mathrm{NO} \nu \mathrm{A}}=810 \mathrm{~km}$ and the average density the neutrino faces before reaching the detector is $\rho_{e}=2.84 \mathrm{~g} / \mathrm{cm}^{3}$ or $n_{e}=6.53 \times 10^{-18} \mathrm{GeV}^{3}$ in the same units of table $3.4 \ldots \ldots \ldots \ldots$. . . . . . . . . . . 78

4.1 The IceCube experiment. . . . . . . . . . . . . . . . . . .

4.2 The pion meson production by a Cosmic Ray proton and the subsequent decay into charged leptons and neutrinos or antineutrinos depending on the lepton produced. Figure taken from $[93] . \ldots \ldots \ldots$

4.3 Energy spectrum of the atmospheric electron and muon neutrino flux as predicted by the HKKM research group [98]. Figure taken from [93]. . . . . . . .

4.4 Energy spectrum of the atmospheric neutrinos and antineutrinos $\nu_{\mu}, \nu_{e}, \bar{\nu}_{\mu}$ and $\bar{\nu}_{e}$ averaged over all directions for the Geographic South Pole. Figure taken from [99]

4.5 Different possible paths that a neutrino can travel through Earth before reaching the IceCube detector. We defined $\theta_{z}=\pi$ for a neutrino which crosses the full Earth diameter . . . . . . . . . . . . . . . . . . . . . . . 
4.6 Number of events observed (black) and predicted using the $\mathrm{N}$ layer model for standard oscillations (red), considering the data set related to the DeepCore subdetector (see section 4.1). The number of events are shown as a function of $\cos \left(\theta_{z}^{\text {reco }}\right)$ in each reconstructed energy bin. . . . . . . . . . . .

4.7 Same as figure 4.7 but considering the data set related to the IceCube detector (see section 4.1). . . . . . . . . . . . . . . . . . . . . . . 94

$4.8 \chi^{2}$ analysis as a function of the Open System parameter, considering the data set related to the DeepCore inner detector (see section 4.1) . . . . . . . . . . . 94

4.9 Same as 4.8 but considering the data set related to the IceCube detector (see section 4.1) . . . . . . . . . . . . . . . . . . . . . . . . 95

4.10 The differences of defining the Dissipator $\mathcal{D}$ in different basis. . . . . . . . . . 99

$4.11 \nu_{\mu}$ survival oscillogram comparing the definition of the Dissipator in mass and matter basis (labeled as Opn). The standard oscillation (labeled as Std) is also shown for reference. The $*$ indicates that the probability was derived following the approach developed in reference [102]. The Open System parameters are fixed to the value $\gamma_{13}=\gamma_{23}=\frac{2}{3} a_{88}=10^{-23} \mathrm{GeV} \ldots \ldots \ldots \ldots 10 \ldots \ldots$

C.1 The Checker map, a useful tool to relate the elements of th Kossakowski matrix with the Dissipator. . . . . . . . . . . . . . . . . . . 121

C.2 Same as figure C.1, but for a three-dimensional system. . . . . . . . . . . 122

D.1 Path defining boundary the angle of the fourth layer. . . . . . . . . . . . 126

D.2 First possible scenario. . . . . . . . . . . . . . . . 127

D.3 Second possible scenario. . . . . . . . . . . . . . . . . . . 128

D.4 Third possible scenario. . . . . . . . . . . . . . . . . . . . . 129

D.5 Possible paths a neutrino can travel in the Earth atmosphere. . . . . . . . . 130 



\section{LIST OF TABLES}

3.1 Relation between the Dissipator and the Kossakowski matrix for each case studied in this section. . . . . . . . . . . . . . . . . . . 59

3.2 Open system parameters used for each case. . . . . . . . . . . . . . . . 61

3.3 Neutrino parameters used for all the analysis in this section. . . . . . . . . . . 61

3.4 Parameters characterizing each Earth layer. The layer radius is written as a fraction of the Earth radius $R_{E}=6371 \mathrm{~km}$ and values for the electron number density $n_{e}$ are based on the Preliminary Earth Reference Model (PREM) [88]. The units are chosen to give the exact numbers we shall use in the time evolution of the neutrino subsystem. . . . . . . . . . . . . . . . .

3.5 Neutrino oscillation parameters, except for the $\delta$ phase, correspond to the best fit values from the NuFIT release [89]. The baseline distance $L$ is fixed to the biggest value possible for the IceCube experiment. $\ldots \ldots \ldots \ldots$. . . . . . .
Open System parameters used for the analysis of the two Dissipators considered

3.6 Open System parameters used for the analysis of the two Dissipators considered
in this section. . . . . . . . . . . . . . . . . 71

4.1 Nuisance parameters along with their associated priors when applicable and the best fit values from analysis $\mathcal{A}$ when fitting the charged and neutral current $\nu_{\tau}$ normalization combined $(\mathrm{CC}+\mathrm{NC})$ and the charged current alone [CC]. Priors are given as central value together with the $\pm 1 \sigma$ ranges when a Gaussian prior is imposed. Table taken from [92], modified to include only the detector related nuisance parameters. . . . . . . . . . . . . . . . . . . 84

4.2 Previous works on neutrino oscillations considering Open System effects. . . . 97 



\section{CONTENTS}

Acknowledgments - Agradecimentos 1

$\begin{array}{ll}\text { Abstract } & 3\end{array}$

$\begin{array}{ll}\text { Resumo } & 5\end{array}$

$\begin{array}{ll}\text { Introduction } & 13\end{array}$

I Theoretical Basis $\quad 19$

1 The Theory Of Neutrino Oscillations 21

1.1 Neutrino oscillations in the plane wave description . . . . . . . . . . . 22

1.2 Neutrino oscillations in the intermediate wave packet description . . . . . . . 23

1.3 Neutrino oscillations in the external wave packet description . . . . . . . . 26

1.3.1 Additional remarks . . . . . . . . . . . . . . . . . . . 32

1.4 Matter effects . . . . . . . . . . . . . . . . . . . . 34

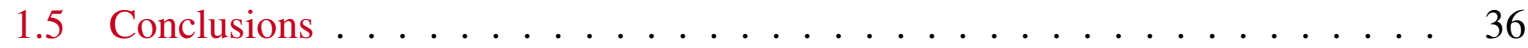

2 The Formalism of Open Quantum Systems 37

2.1 Density Operators . . . . . . . . . . . . . . . . . . . . . . . . . . . . 39

2.1.1 Time Evolution . . . . . . . . . . . . . . . . . . . 40

2.1.2 Composite systems and the partial Trace . . . . . . . . . . . 41

2.2 Open Quantum Systems ～. . . . . . . . . . . . . . . . . . 42

2.2.1 Dynamical maps ......................... 43

2.2.2 Markovian processes and the Quantum Master Equation . . . . . . . . 45

2.2 .3 Quantum Entropies . . . . . . . . . . . . . . . . 50

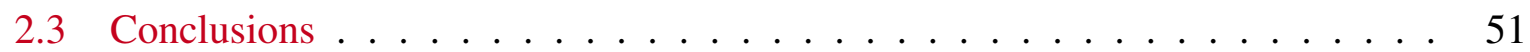


3 Open System effects in neutrino oscillations $\quad \mathbf{5 5}$

3.1 A general two-dimensional example . . . . . . . . . . . . . . 56

3.2 Neutrino oscillations in two generations with Open System effects . . . . . . . 58

3.2.1 Average energy conservation analysis . . . . . . . . . . . . 58

3.2.2 Discussion of Open System effects in two generations . . . . . . . . . 60

3.3 Neutrino oscillations in three generations with Open System effects . . . . . 66

3.4 Conclusions . . . . . . . . . . . . . . . . . . 79

4 IceCube atmospheric neutrinos in an Open System approach 81

4.1 The IceCube experiment . . . . . . . . . . . . . . . . . 82

4.2 Atmospheric neutrinos . . . . . . . . . . . . . . . . 85

4.3 The $\mathrm{N}$ layer model . . . . . . . . . . . . . . . . . . . . . . 87

4.4 Calculation of atmospheric neutrino events in the IceCube experiment . . . . 89

4.5 Statistical analysis . . . . . . . . . . . . . . . . . . . 9 91

4.6 Discussion of the results . . . . . . . . . . . . . . . . . 92

4.7 Comments of previous works . . . . . . . . . . . . 96

$\begin{array}{ll}\text { Conclusions and future perspectives } & 103\end{array}$

A Neutrino oscillations in different prescriptions $\quad 109$

A.1 Oscillations in the internal wave packet description . . . . . . . . . . . . 109

A.2 Oscillations in the external wave packet description . . . . . . . . . . . 112

A.2.1 The Grimus-Stockinger theorem: . . . . . . . . . . . . . . 115

B Analytical results for Open Systems 117

B.1 A sufficient condition for the entropy to increase . . . . . . . . . . . . . 117

B.2 The two-dimensional Lindbladian . . . . . . . . . . . . . . . . 118

C The Checker map for the two- and three-dimensional Dissipator 121

C.1 Mapping the two-dimensional Dissipator . . . . . . . . . . . . . . . . . 121

C.2 Mapping the three-dimensional Dissipator . . . . . . . . . . . . . . . 122

C.3 Components of the three dimensional Dissipator . . . . . . . . . . . . . 122

D Calculation of the distance a neutrino travel through each Earth layer 125

D.1 Possible paths travelled by a neutrino through the Earth . . . . . . . . . . 125

D.1.1 The boundaries angles . . . . . . . . . . . . . . 125

D.1.2 First possibility . . . . . . . . . . . . . . 126

D.1.3 Second possibility . . . . . . . . . . . . . . 127

D.1.4 Third possibility . . . . . . . . . . . . . . . 128

D.1.5 Distance travelled through the atmosphere . . . . . . . . . . 130 


\section{INTRODUCTION}

\section{"Neutrino physics is largely an art of learning a great deal by observing nothing."}

(Haim Harari)

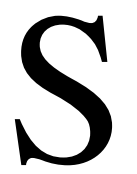

INCE the radioactive ladies and gentleman heard for the first time of the neutron hypothesis [1] we have improved a lot what we know about the microscopic world. Neutrinos have become one of the building blocks of the best theory of particles we have so far, the Standard Model. In order to reach the knowledge we have today we had to overcome many theoretical an experimental difficulties. Along the way we were able to learn many of the peculiarities that make this particle unique.

If we tell anyone that $10^{21}$ neutrinos had crossed their body during the past two years without being even noticed, the immediate reaction would be rejection. The fact that we, as physicists, know about the existence of a neutrino and are able to calculate how many crossed our bodies during the time this work took to be completed does not make the situation more comfortable. When the subject is neutrinos, questions are much more recurrent than answers. The history behind the progress in understanding these elusive particles is a very good example of how Science evolves, being its path is full of adversities and dead ends. The attempts to unravel the deepest secrets of nature have turned Science as the most successful enterprises ever made by the human being.

We can trace back in time the first stages of the neutrino hypothesis. In the early 1900's, a lot of scientists were facing difficulties to understand the behavior of beta decay. Their models of the atomic nucleus led to the belief that an electron emitted in a radioactive decay should have a unique energy. During thirty years [2], different experiments were in contradiction, and also the physics community did not have a uniform opinion. Only after the work of J. Chadwick and Hans Geiger [3] the conclusion became reliable: electrons are emitted with several energies. 
Introduction

The continuous spectra of beta decay had become a fact. In an attempt to explain this unexpected behavior, Niels Bohr was willing to give up energy conservation in quantum theories, a rather radical proposal. To avoid such a dramatic change Pauli proposed in a letter [1] "as a desperate remedy" the existence of the neutron, a massless fermion which would inhabit the atomic nucleus and be emitted along with the electron ${ }^{1}$, balancing the energy conservation and justifying the continuity of the spectra. In that letter, the neutrino was born. The best description of a ghost particle we have so far.

Fermi not only took Pauli's proposal seriously but also elevated the status of the neutrino, as he introduced a new kind of force, the weak interaction, which can only happen if the tiny particle is part of the real physical world. He changed the atomic picture by assuming that the nucleus consists of only protons and neutrons. Here it is important to assert that these neutrons are not Pauli's particle but the one hypothesized by Rutherford and later discovered by Chadwick [4]. Since Chadwick's neutron was much heavier than Pauli's, in order to distinguish both Fermi called the second neutrino an abbreviation for the little neutral one. This revolutionized the theory of beta decay: in Fermi's view the neutron would turn into a proton by emitting together a neutrino and an electron. Also, the nucleus formed by neutrons and protons solved the spin-statistics problem [2]. This was such a dramatic change that Fermi's paper was rejected by Nature [5] because "it contained speculations too remote from reality to be of interest to the reader". Furthermore, not all experimental evidence was in agreement with Fermi's theory, an alternative hypothesis was proposed by Emil Konopinski and George Uhlenbeck which could better fit the available results [2]. It turned out that both the experiments and the experimenttheory comparisons were wrong. Fermi theory of beta decay prevailed. It was the first attempt to explain the weak interaction and the first step towards the Standard Model of electroweak interactions we have today.

It took some twenty years after that to establish the correct form of the interaction, among the five possible relativistic invariant forms: scalar (S), tensor (T), vector (V), axial-vector (A) and pseudoscalar $(\mathrm{P})$. The right combination emerged only after experiments had confirmed the T. D. Lee and C. N. Yang hypothesis of parity nonconservation in weak interaction processes [6].

In the meantime, Reines and Cowan [7, 8], two members of the Los Alamos weapons laboratory, were joining efforts to see the invisible. Their project, named poltergeist, was the first attempt to detect the neutrino. The first proposal consisted of detecting the neutrinos produced in a nuclear explosion. This experiment was never performed, as J. M. B. Kellog, the director of the Physics Division at Los Alamos, suggested to detect neutrinos produced in fission reac-

\footnotetext{
${ }^{1}$ At that time the electron was assumed to be part of the nucleus to solve the spin-statistics problem in the atomic nuclei.
} 
tors instead. Despite the reduced flux, the reactor experiment could run for years uninterrupted. Their first data had a huge background contamination leading to an inconclusive result. Only after they had improved the experimental setup a second trial started. Neutrinos were produced by the Savannah River nuclear reactor, then they penetrated the three partitioned detectors with two targets composed of plastic tanks filled with 200 liters of water with cadmium chloride dissolved. Neutrinos would induce an inverse beta decay process

$$
\bar{\nu}+p \rightarrow n+e^{+},
$$

then positrons would be annihilated by electrons present in the water and the neutron captured by the dissolved cadmium. The annihilation results in two gamma rays which could be detected in prompt coincidence; if that signal was followed by another light signal consistent with the time interval characteristic of neutron capture, a neutrino event would have happened. The telegram sent by Cowan and Reines to Pauli after the completion of the experiment illustrates the conclusion [2]:

"We are happy to inform you that we have definitely detected neutrinos from fission fragments by observing the inverse beta decay of protons. Observed cross-section agrees well with expected six times 10 to minus forty-four square centimeters"

The neutrino was detected. They started the process of changing the role the neutrino plays in our lives, from the missing piece in a puzzle to a tool by which we can study Nature. The adventure had just started.

We know neutrinos carry information about different kinds of events. They were present in the first stages of the universe and because of the feebleness of their interactions they have carried all the secrets about the beginning of time almost unchanged. The knowledge about the inner part of stars is not accessible using photons, promoting neutrinos to be good candidates to make further progress in understanding the most violent events that have ever happened in the universe. The Sun, which makes life on Earth possible, is powered by chain reactions involving neutrinos. The absence of this elusive particle that at first sight may seem harmless, has dramatic consequences.

Bahcall and Davis [9] went on the quest to observe solar neutrinos. The detection of those particles was important to provide tests of the Standard Solar Model (SSM), the theoretical framework describing the Sun evolution. Furthermore, the SSM was also used to study the properties of other stars very different from the Sun, a problem with the solar model could have consequences in different branches of physics. Ray Davis followed Pontecorvo's suggestion [10] and built a neutrino target of 380 cubic meters filled with 3800 liters carbon tetrachloride, 
placed 1478 meters underground in the Homestake Gold Mine. The electron neutrino would induce the transmutation of the chlorine atom

$$
{ }^{37} \mathrm{Cl}+\nu_{\mathrm{e}} \rightarrow{ }^{37} \mathrm{Ar}+\mathrm{e}^{-},
$$

then by counting the amount of Argon atoms produced the number of neutrino events could be recovered. The results were in contradiction with the theoretical expectation [9], the problem became more evident when different solar neutrino experiments reached the same conclusion [11, $12,13,14,15]$. Since in this scenario the problem could be related to neutrino physics, faulty astrophysics, or even both, different solutions were proposed. Bahcall tested more than 1001 different modifications of the SSM and also considered different effects that might change the flux of neutrinos reaching the Earth, such as neutrino decay, the Mikheyev-Smirnov-Wolfenstein (MSW) effect, strong solar magnetic field, and WIMPS [9].

Among the possible explanations, the MSW effect was the most theoretically appealing, since it did not introduce new particles nor any changes in the properties of the Sun. This effect required a rather small, but nonzero, mass for the neutrino, furthermore, it implied that the neutrinos could change their flavor while finding their way out of the Sun. Since the reaction (2) is sensitive only to $\nu_{e}$ the experiment would never measure all the neutrinos coming from the Sun. The independent confirmation in atmospheric $[16,17,18,19,20,21,22]$ neutrino experiments, strengthened the oscillation hypothesis. In fact, we have to wait until the SNO experiment [23], which could detect all the different neutrino flavors, to explain the discrepancy. After that, the oscillation hypothesis have also been confirmed using neutrinos produced in nuclear reactors [24, 25, 26, 27, 28] and accelerator [29, 30, 31, 32, 33] experiments. The total flux was indeed compatible with Bahcall's calculations, the problem again was regarding with our understanding about the neutrinos: they can oscillate.

This discovery resulted in very interesting questions. The absolute neutrino mass scale is still unknown and also since the Standard Model of particles lacks a mass term for neutrinos, we still do not even know how they acquire such a small mass. Furthermore, the neutrino is the only known fermion that can be either a Majorana particle, which means that it would be indistinguishable from its antiparticle or a Dirac particle, being then different from its anti partner. The first experimental evidence favored the second option [2], but ironically parity violation made the result ambiguous. Different experiments have been proposed since then, but hitherto the nature of neutrinos is unknown.

In summary, the neutrino was born as a dramatic proposal to avoid an even more dramatic assumption. It has become part of our arsenal to study Nature in different contexts, as an unusual example, a neutrino tomography of the Earth was explored in [34]. The rather particular 
properties of neutrinos have made them difficult to unravel, but at the same time have made them very special. They are possibly the only manifestation of beyond Standard Model physics we know so far. They are also the most elementary quantum interferometer we have in Nature.

The fact that neutrinos undergo flavor oscillations, which is an intrinsically quantum effect, has stimulated us to use them as a tool to study the quantum realm. We will explore the possibility that neutrino oscillations can be described as an Open Quantum System and that the neutrino oscillation pattern can be affected by unknown interactions with the Environment which can be probed by neutrino oscillation data.

For this purpose, in chapter one, we review the theoretical basis of neutrino oscillations in vacuum considering different descriptions, starting with the most used, but theoretically and physically inconsistent, the plane wave treatment, and then evolving to a Quantum Field Theoretical framework. After that we show how to take into account matter effects in neutrino oscillations in preparation for the next chapters.

In chapter two we explain the Open Quantum System formalism. It consists of considering the neutrino as a subsystem immersed in a larger system, which we will call the Environment. This description is theoretically appealing because it does not require knowledge about what might interact with the neutrino subsystem to make predictions. Besides the general formalism, we also discuss useful tools to help us understand the Open System effects, such as quantum entropy and the Checker map. The second is a map we have developed to help us in the task of finding interesting Open Quantum System scenarios to confront with experiments.

Then in chapter three, we use the theoretical framework developed in chapters one and two, to study neutrino oscillations in two and three generations. The two generation description is a good starting point due to its simplicity. But even in two generations in vacuum we can find interesting new features when Open Quantum System effects are present.

After that, we move to the more realistic description of neutrino oscillations in three generations with matter effects. In this scenario we see that Open System effects have interesting consequences, not only on the oscillation pattern but also in the number of relevant phases in the neutrino oscillations. Finally, to conclude the chapter we compare the reach of the IceCube with the $\mathrm{NO} \nu \mathrm{A}$ experiment to Open Quantum System effects.

Motivated by the results of previous chapter, in chapter four we finally tackle the challenging problem of simulating predictions for the atmospheric neutrinos data in IceCube. In order to do that a detailed discussion about the experiment, the data sets, the computation of probabilities and number of events and the statistical analysis is provided. After that we are finally in position to compare our prediction with the data released by the IceCube collaboration. We present our results for a specific Open Quantum System scenario and compare them with previous works. 
Finally, we conclude this work by summarizing our main results and discussing future perspectives. 


\section{Part I}

\section{Theoretical Basis}





\section{CHAPTER 1}

\section{THE THEORY OF NEUTRINO OSCILLATIONS}

\section{"They are the most tiny quantity of reality ever imagined by a human being."}

(F. Reines)

$\mathrm{T}$

HE fact that flavor states could be a superposition of states with definite mass was first proposed by Pais and Piccioni [35]. This idea was used by Potencorvo in 1957 to present the hypothesis of neutrino-antineutrino oscillation [36]. After that neutrino oscillation was suggested in the context of two generations by Maki, Nakagawa, and Sakata [37]. The observation of a deficit in the flux of solar neutrinos detected by the Homestake Mine experiment [38, 39] and confirmed by other experiments, for example, SAGE [11], GALLEX [13, 40], GNO [12], Kamiokande-II [15, 41] and Super-Kamiokande [14, 42] was the first signal that something weird might be happening in the neutrino sector. Since at the time it could also be a problem with the standard solar model [9], neutrinos were found guilty only after independent confirmation by experiments detecting neutrinos produced in the atmosphere $[16,17,18,21$, $19,20,22]$, in nuclear reactors [28, 27, 24, 26, 25] and in accelerators [29, 30, 31, 32, 33]. The theoretical basis behind the oscillation phenomena has been seriously questioned since then [43, $44,45,46,47]$.

In this chapter, we want to discuss not only the reason why neutrinos oscillate but also under which conditions a particle can undergo this phenomenon. For that purpose, we will review the development of the theory of neutrino oscillations starting with the standard and most used plane wave approach. Then we will replace plane waves by wave packets, the so-called intermediate 
wave packet model, and, finally, we will study the Quantum Field theoretical approach, the most complete theoretical framework to describe particles we have until the present moment. Our objective here is to analyze neutrino oscillations focusing on the formalism, so the study of vacuum oscillations is enough to discuss all the important points. We will assume this to be the case for most of the chapter. Only at the end we will show how matter effects can change the time evolution. The reader who wants to see the Quantum Field Theoretical approach here exposed considering matter effects, is invited to see [48].

\subsection{Neutrino oscillations in the plane wave description}

In this formalism the flavor eigenstate is represented as a linear combination of the mass eigenstates which are assumed to evolve in time as a plane wave ${ }^{1}$

$$
\left|\nu_{\alpha}\right\rangle=\sum_{i=1}^{3} U_{\alpha i}^{*}\left|\nu_{i}\right\rangle
$$

where Greek and Latin indexes label flavor and mass states, respectively, and $U$ is the PontercorvoMaki-Nakagawa-Sakata (PMNS) matrix. The fact that it is $U^{*}$ that enters equation (1.1) is inherited from Quantum Field Theory, once the field responsible for creating a particle is $\bar{\nu}$. This is interesting because, as we will demonstrate, the Quantum Field Theoretical description predicts that no oscillations would occur in the plane wave limit.

The Hamiltonian of the system is given, neglecting a global factor, by

$$
H=\left(\begin{array}{ccc}
\frac{m_{1}^{2}}{2 E} & 0 & 0 \\
0 & \frac{m_{2}^{2}}{2 E} & 0 \\
0 & 0 & \frac{m_{3}^{2}}{2 E}
\end{array}\right),
$$

where $m_{i}(i=1,2,3)$ are the neutrino masses and $E$ their energy. We have assumed neutrinos to be ultra-relativistic particles with equal energies. Since the Hamiltonian is diagonal in the mass basis, the evolution is trivial

$$
\left|\nu_{\alpha}(t)\right\rangle=\sum_{i=1}^{3} U_{\alpha j}^{*}\left|\nu_{j}(t)\right\rangle=\sum_{j=1}^{3} U_{\alpha j}^{*} e^{-\frac{i m_{j}^{2}}{2 E} t}\left|\nu_{j}\right\rangle .
$$

The oscillation probability of an $\alpha$ flavored neutrino $\nu_{\alpha}$ to change into $\nu_{\beta}$ after time $t$ is then given by

\footnotetext{
${ }^{1}$ In this dissertation we will use the natural system of units, so from now on we will set $\hbar=c=1$.
} 


$$
\mathcal{P}_{\nu_{\alpha} \rightarrow \nu_{\beta}}=\left|\left\langle\nu_{\beta}(t) \mid \nu_{\alpha}(0)\right\rangle\right|^{2}=\sum_{i, j=1}^{3} U_{\beta j} U_{\beta i}^{*} U_{\alpha j}^{*} U_{\alpha i} e^{-\frac{i \Delta m_{i j}^{2}}{2 E} t},
$$

this is the standard result. But there are subtleties $[43,49]$ a few of them which we will solve in the next sections are:

(a) How can we define detection and production processes if a plane wave is completely delocalized $^{2}$ ?

(b) Why should the energy of all eigenstates be the same?

\subsection{Neutrino oscillations in the intermediate wave packet de- scription}

Almost twenty years after Pontecorvo's seminal paper, Nussinov [50], for the first time, proposed the use of wave packets instead of plane waves. After that, Kayser [46] studied conditions for oscillations to happen and in 1991, Giunti, Kim, and Lee [47] provided the first complete derivation of the oscillation probability in the intermediate wave packet model.

Since then, this approach has been used in different contexts and the probabilities have been derived under different assumptions [49, 51, 52, 53]. Its limitations have become evident as well $[43,49]$.

Here we will review how to obtain the probabilities in this framework. In the intermediate wave packet description, the neutrino mass eigenstate is treated as a wave packet

$$
\left|\nu_{j}\right\rangle=\int[d p] f_{\nu_{j}}(\overrightarrow{\mathrm{p}}, \overrightarrow{\mathrm{P}})\left|\nu_{j}, \overrightarrow{\mathrm{p}}\right\rangle
$$

where the index $j$ still labels a mass eigenstate and $f_{\nu_{j}}(\overrightarrow{\mathrm{p}}, \overrightarrow{\mathrm{P}})$ is the momentum distribution function around the mean momentum $\overrightarrow{\mathrm{P}}$. Also we have used the shorthand notation

$$
[d p] \equiv \frac{d^{3} \overrightarrow{\mathrm{p}}}{(2 \pi)^{3} \sqrt{2 E(\mathrm{p})}} .
$$

The normalization condition is given by

$$
\left\langle\nu_{i}, \overrightarrow{\mathrm{p}}^{\prime} \mid \nu_{j}, \overrightarrow{\mathrm{p}}\right\rangle=2 E_{j}(\mathrm{p})(2 \pi)^{3} \delta^{3}\left(\tilde{\mathrm{p}}-\tilde{\mathrm{p}}^{\prime}\right) \delta_{\mathrm{ij}}
$$

which establishes the normalization of the distribution function,

\footnotetext{
${ }^{2}$ It is, by definition, a state of definite momentum.
} 


$$
\int \frac{d^{3} \overrightarrow{\mathrm{p}}}{(2 \pi)^{3}}\left|f_{\nu_{j}}(\overrightarrow{\mathrm{p}}, \overrightarrow{\mathrm{P}})\right|^{2}=1
$$

The pure $\alpha$ flavored state can then be defined as ${ }^{3}$

$$
\rho_{\alpha}=\sum_{i, j} U_{\alpha i}^{*} U_{\alpha j} \int[d p] \int\left[d p^{\prime}\right] f_{\nu_{i}}(\overrightarrow{\mathrm{p}}, \overrightarrow{\mathrm{P}}) f_{\nu_{j}}^{*}(\overrightarrow{\mathrm{p}}, \overrightarrow{\mathrm{P}})\left|\nu_{i}, \overrightarrow{\mathrm{p}}\right\rangle\left\langle\nu_{j}, \overrightarrow{\mathrm{p}}^{\prime}\right| .
$$

The evolved neutrino state produced in the position $\overrightarrow{\mathrm{x}}=\overrightarrow{\mathrm{x}}_{P}$ at the instant $t=t_{P}$ is given by

$$
\begin{array}{r}
\rho_{\alpha}(t)=\sum_{i, j} U_{\alpha i}^{*} U_{\alpha j} \int[d p] \int\left[d p^{\prime}\right] f_{\nu_{i}}(\overrightarrow{\mathrm{p}}, \overrightarrow{\mathrm{P}}) f_{\nu_{j}}^{*}(\overrightarrow{\mathrm{p}}, \overrightarrow{\mathrm{P}}) \\
\times e^{-i\left(E_{i}-E_{j}\right)\left(t-t_{p}\right)+i\left(\overrightarrow{\mathrm{p}}-\overrightarrow{\mathrm{p}}^{\prime}\right) \cdot \overrightarrow{\mathrm{x}}_{P}}\left|\nu_{i}, \overrightarrow{\mathrm{p}}\right\rangle\left\langle\nu_{j}, \overrightarrow{\mathrm{p}}^{\prime}\right| .
\end{array}
$$

After production the neutrino evolves until the detection position $\overrightarrow{\mathrm{x}}=\overrightarrow{\mathrm{x}}_{D}$ at time $t=t_{D}$. The oscillation probability can then be calculated through

$$
\mathcal{P}_{\nu_{\alpha} \rightarrow \nu_{\beta}}=\operatorname{Tr}\left\{\rho_{\beta}\left(t_{D}\right) \rho_{\alpha}\left(t_{P}\right)\right\}
$$

To make further progress three assumptions are necessary:

1. The momentum distribution function $f_{\nu_{j}}(\overrightarrow{\mathrm{p}}, \overrightarrow{\mathrm{P}})$ is assumed to be sharply peaked at the mean momentum $\overrightarrow{\mathrm{P}}$. Then we will approximate it by a Gaussian function in momentum space with mean momentum $\overrightarrow{\mathrm{P}}$ and momentum width $\sigma_{p, i}{ }^{4}$;

2. The biggest energy difference between neutrino mass eigenstates is small when compared to the energy at mean momentum $\overrightarrow{\mathrm{P}}$;

3. The time of production $t_{P}$ and detection $t_{D}$ are not measured;

The result is ${ }^{5}$

$$
\mathcal{P}_{\nu_{\alpha} \rightarrow \nu_{\beta}} \propto \sum_{i, j} U_{\beta i}^{*} U_{\beta j} U_{\alpha j}^{*} U_{\alpha i} \exp \left(-2 \pi i \frac{L}{L_{i j}^{\mathrm{osc}}}-\left(\frac{L}{L_{i j}^{\mathrm{coh}}}\right)^{2}-2 \pi^{2}\left(\frac{\sigma_{x}}{L_{i j}^{\mathrm{osc}}}\right)^{2}\right)
$$

\footnotetext{
${ }^{3}$ We will use the density operator. For a pure state, it is defined to be $\rho_{\alpha}(t)=\left|\nu_{\alpha}(t)\right\rangle\left\langle\nu_{\alpha}(t)\right|$. The details concerning this operator are shown in chapter [2].

${ }^{4}$ The label $i$ is necessary to distinguish between production and detection.

${ }^{5}$ See appendix A for the details.
} 
Where

$$
L_{i j}^{\mathrm{osc}} \equiv \frac{4 \pi E}{\Delta m_{i j}^{2}},
$$

and

$$
L_{i j}^{\mathrm{coh}} \equiv \frac{4 \sqrt{2} E^{2}}{\left|\Delta m_{i j}^{2}\right|} \sigma_{x}
$$

are the oscillation and coherence length, respectively.

The improvements of this formalism are the last two terms in (1.12), the coherence length given by (1.14) defines a distance beyond which the coherence of the wave packet is lost. This term arises because the group velocities $v_{i}$ are different which causes the different wave packets to separate during the propagation. The last term in (1.12) suppresses the oscillations if $L_{i j}^{\text {osc }}$ is comparable to the localization width $\sigma_{x}$ of the wave packet. The immediate consequence is that an experiment, aiming to measure neutrino masses, will unavoidably destroy the oscillation pattern. The capability to distinguish between mass eigenstates implies that only one state is detected [46].

It is very important to emphasize that the decoherence we have just described here is different from the one we will discuss in the Open System framework. Here coherence can be recovered by the experimental setup when we take into account its finite exposition time. If the width of this time interval is bigger than the wave packets separation, the experiment will measure them as a superposition, and coherence is restored.

In equation (1.12) we have shown only the part of the final result which can be interpreted as an oscillation probability. The final expression is not properly normalized and it is necessary to impose this condition by hand at the end of the calculation. This cannot be remedied by any modification [49] and it is a consequence of the fact that production and detection are not properly taken into account in the intermediate wave packet model. In a consistent framework, we should obtain the final result already normalized, this being the first issue with this description.

Despite the improvements when compared to the plane wave framework, the intermediate wave packet model cannot answer a great deal of questions [45]. Also, the fact that the oscillating particle is never directly measured enforces the need of calculating the transition probability involving only observable particles that participate in the production and detection processes. 


\title{
1.3 Neutrino oscillations in the external wave packet descrip- tion
}

The external wave packet model was discussed in many works $[44,45,54,55,56,57,58$, $59,60,61]$, part of them are relatively new and, in particular, reference [60] is the latest review released about the subject up to the date of this work. This fact reinforces that, while neutrino oscillation is a well-established phenomenon, its theoretical basis is still not fully understood. Akhmedov that has extensively worked on the quest for a complete theory of neutrino oscillations said [49]:

\begin{abstract}
"Though quite mature, the theory of neutrino oscillations is in my opinion far from being closed. Over the years, many times it had appeared to us to be complete and finished, but each time this turned out to be wrong. I believe that we are still in the same situation now."
\end{abstract}

The present description is not only a theoretical improvement but also has experimental consequences, it was already used to discuss oscillations of Mossbauer neutrinos [62] and as a viable explanation to the reactor anti-neutrino anomaly [63]. Oscillation phenomenon is intrinsically a quantum mechanical effect, the more we understand it the more we will appreciate the quantum theory and test its foundations.

Here we will follow mostly the references [49, 45]. In [45] the author discussed all the possible scenarios for neutrino oscillations considering a Quantum Field Theoretical framework with external particles described by a Gaussian wave packet and in [49] the author used how probabilities are calculated in Quantum Field Theory to discuss the limits into which we can recover (1.4) considering the calculations developed in [45].

In the Quantum Field Theoretical approach, we will analyze the neutrino production, propagation, and detection as a single process, with the neutrino being the intermediate state (see figure 1.1)

The external states are described by wave packets ${ }^{6}$

\footnotetext{
${ }^{6}$ Hence the name external wave packets.
} 


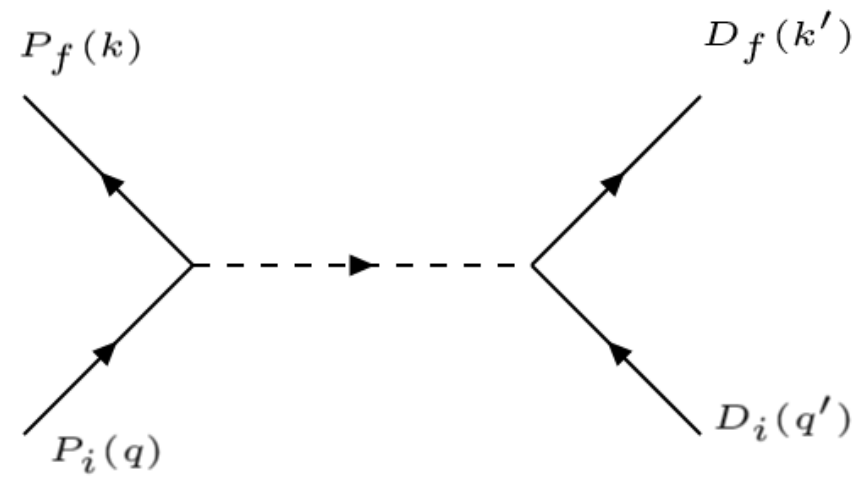

Figure 1.1: Feynman diagram representing the process.

$$
\begin{aligned}
\left|P_{i}\right\rangle & =\int[d q] f_{P, i}(\overrightarrow{\mathrm{q}}, \overrightarrow{\mathrm{Q}})\left|P_{i}, q\right\rangle, \\
\left|P_{f}\right\rangle & =\int[d k] f_{P, f}(\overrightarrow{\mathrm{k}}, \overrightarrow{\mathrm{K}})\left|P_{f}, k\right\rangle, \\
\left|D_{i}\right\rangle & =\int\left[d q^{\prime}\right] f_{D, i}\left(\overrightarrow{\mathrm{q}}^{\prime}, \overrightarrow{\mathrm{Q}}^{\prime}\right)\left|D_{i}, q^{\prime}\right\rangle, \\
\left|D_{f}\right\rangle & =\int\left[d k^{\prime}\right] f_{D, f}\left(\overrightarrow{\mathrm{k}}^{\prime}, \overrightarrow{\mathrm{K}}^{\prime}\right)\left|D_{f}, k^{\prime}\right\rangle .
\end{aligned}
$$

The states are assumed to satisfy the normalization conditions (1.7), (1.8). The amplitude of the Feynman diagram (1.1) is given by

$$
i \mathcal{A}_{\alpha \beta}=\left\langle P_{f} D_{f}\left|\mathrm{~T}\left\{\exp \left[-\mathrm{i} \int \mathrm{d}^{4} x H_{I}(x)\right]\right\}-\mathbb{1}\right| P_{i} D_{i}\right\rangle
$$

which encompasses the whole process, production, propagation, and detection. $\mathrm{T}\{\}$ is the time ordering operator and $H_{I}$ is the Hamiltonian in the interaction picture. If the external particles were described by plane waves, the second-order expansion of (1.16) would result in

$$
i \mathcal{A}_{\alpha \beta}=\sum_{j} U_{\alpha j}^{*} U_{\beta j} i \mathcal{A}_{j}^{\text {p.w. }}\left(q, k ; q^{\prime}, k^{\prime}\right)
$$

where $\mathcal{A}_{j}^{\text {p.w. }}\left(q, k ; q^{\prime}, k^{\prime}\right)$ is the plane wave amplitude for the process, which can be calculated by the usual methods of Quantum Field Theory [64] and $j$ is the mass state label. Considering again $\overrightarrow{\mathrm{x}}_{P}$ and $\overrightarrow{\mathrm{x}}_{D}$ as the production and detection positions, respectively, we have that 


$$
\begin{aligned}
\mathcal{A}_{j}^{\text {p.w. }}\left(q, k ; q^{\prime}, k^{\prime}\right)= & \int d^{4} x_{1} \int d^{4} x_{2} \tilde{M}_{D}\left(q^{\prime}, k^{\prime}\right) e^{-i\left(q^{\prime}-k^{\prime}\right)\left(x_{2}-x_{D}\right)}, \\
& \times i \int \frac{d^{4} p}{(2 \pi)^{4}} \frac{\not p+m_{j}}{p^{2}-m_{j}^{2}+i \epsilon} e^{-i p\left(x_{2}-x_{1}\right)} \tilde{M}_{P}(q, k) e^{-i(q-k)\left(x_{1}-x_{P}\right)},
\end{aligned}
$$

where $\tilde{M}_{D}$ and $\tilde{M}_{P}$ are the matrix elements associated with detection and production vertices, respectively (see appendix A for the details concerning the calculations of this section). Changing variables

$$
\begin{aligned}
& x_{1}=x_{P}+x_{1}^{\prime}, \\
& x_{2}=x_{D}+x_{2}^{\prime},
\end{aligned}
$$

using the spinor completeness relation $\sum_{s}=u_{j}(p, s) \bar{u}_{j}(p, s)=\not p+m_{j}$, and redefining

$$
\begin{aligned}
M_{P, j}(q, k) & =\frac{\bar{u}_{j L}(p)}{\sqrt{p_{0}}} \tilde{M}_{P}\left(q^{\prime}, k^{\prime}\right), \\
M_{D, j}\left(q^{\prime}, k^{\prime}\right) & =\tilde{M}_{D}\left(q^{\prime}, k^{\prime}\right) \frac{u_{j L}(p)}{\sqrt{p_{0}}},
\end{aligned}
$$

where the index $L$ is due to the fact that neutrinos are left-handed states, we can rewrite equation $(1.17)$

$$
\begin{aligned}
\mathcal{A}_{j}^{\text {p.w. }}\left(q, k ; q^{\prime}, k^{\prime}\right)= & i \int \frac{d^{4} p}{(2 \pi)^{4}} \frac{e^{-i p\left(x_{D}-x_{P}\right)}}{p^{2}-m_{j}^{2}+i \epsilon} \int d^{4} x_{1}^{\prime} \sqrt{p_{0}} M_{P, j}(q, k) e^{-i(q-k-p) x_{1}^{\prime}} \\
& \times \int d^{4} x_{2}^{\prime} \sqrt{p_{0}} M_{D, j}\left(q^{\prime}, k^{\prime}\right) e^{-i\left(q^{\prime}-k^{\prime}+p\right) x_{2}^{\prime}}
\end{aligned}
$$

The only change when the external states are described by wave packets is the introduction of the distribution functions when the second quantized field acts, for example, on the initial state at the production in (1.15). It will generate the new factor ${ }^{7} \int[d p] f_{P, i}(\overrightarrow{\mathrm{q}}, \overrightarrow{\mathrm{Q}})$. Then the full amplitude is given by

$$
i \mathcal{A}_{\alpha \beta}=\sum_{j} \int \frac{d^{4} p}{(2 \pi)^{4}} U_{\alpha j}^{*} U_{\beta j} \phi_{P, j}\left(p^{0}, \overrightarrow{\mathrm{p}}\right) \phi_{D, j}\left(p^{0}, \overrightarrow{\mathrm{p}}\right) \frac{2 p_{0} e^{-i p_{0} T+i \overrightarrow{\mathrm{p}} \cdot \overrightarrow{\mathrm{L}}}}{p^{2}-m^{2}+i \epsilon},
$$

\footnotetext{
${ }^{7}$ It is analogous to the polarization factor $\epsilon^{\mu}(k)$ which arises when a photon is created.
} 
where

$$
\begin{aligned}
& \phi_{P, j}\left(p_{0}, \overrightarrow{\mathrm{p}}\right)=\int d^{4} x_{1}^{\prime} e^{i p x_{1}^{\prime}} \int[d q] \int[d k] f_{P, i}(\overrightarrow{\mathrm{q}}, \overrightarrow{\mathrm{Q}}) f_{P, f}^{*}(\overrightarrow{\mathrm{k}}, \overrightarrow{\mathrm{K}}) e^{-i(q-k) x_{1}^{\prime}} M_{P, j}(q, k), \\
& \phi_{D, j}\left(p_{0}, \overrightarrow{\mathrm{p}}\right)=\int d^{4} x_{2}^{\prime} e^{-i p x_{2}^{\prime}} \int\left[d q^{\prime}\right] \int\left[d k^{\prime}\right] f_{D, i}\left(\overrightarrow{\mathrm{q}}^{\prime}, \overrightarrow{\mathrm{Q}}^{\prime}\right) f_{D, f}^{*}\left(\overrightarrow{\mathrm{k}}^{\prime}, \overrightarrow{\mathrm{K}}^{\prime}\right) e^{-i\left(q^{\prime}-k^{\prime}\right) x_{1}^{\prime}} M_{D, j}\left(q^{\prime}, k^{\prime}\right) .
\end{aligned}
$$

Quantum field theory predicts matrix elements which are integrated over the phase space to obtain cross-sections for possible events. At this point it is necessary to review these concepts to interpret the meaning of oscillation probability in this context.

The cross-section for a particular process can be calculated by

$$
d \sigma=\frac{1}{T} \frac{1}{\Phi} d P
$$

where $T$ is the characteristic time of the experiment, $\Phi$ is the flux ${ }^{8}$ and $P$ is the probability predicted in the Quantum Field Theoretical framework ${ }^{9}$. For a detector sensitive to the production of the charged lepton $\beta$ we can define the overall process detection rate as

$$
\Gamma_{\alpha \beta}^{\mathrm{tot}}=\int d E \frac{1}{4 \pi L^{2}} \frac{d \Gamma_{\alpha}^{\mathrm{prod}}(E)}{d E} P_{\alpha \beta}(L, E) \sigma_{\beta}(E),
$$

where $L$ is the distance between the source, which produces leptons of flavor $\alpha$, and the detector. The factor $d \Gamma_{\alpha}^{\text {prod }}(E) / d E$ is the spectral distribution at emission of neutrinos of flavor $\alpha, \sigma_{\beta}$ is the cross-section related to the detection of a $\beta$ flavored neutrino and $P_{\alpha \beta}(L, E)$ is the oscillation probability of a $\alpha$ flavored neutrino to change into a $\beta$ flavored one. We have assumed the emission to be spherically symmetric which leads to the factor $4 \pi L^{2}$ in the denominator. The oscillation probability is then defined as

$$
P_{\alpha \beta}(L, E)=\frac{d \Gamma_{\alpha \beta}^{\mathrm{tot}}(E) / d E}{\frac{1}{4 \pi L^{2}}\left\{d \Gamma_{\alpha}^{\mathrm{prod}}(E) / d E\right\} \sigma_{\beta}(E)} .
$$

To calculate the overall production rate is necessary to integrate (1.21), using the GrimusStockinger theorem [65] the result is (see appendix A)

$$
i \mathcal{A}_{\alpha \beta}=-\frac{i}{8 \pi^{2} L} \sum_{j} U_{\alpha j}^{*} U_{\beta j} \int d E \phi_{P, j}\left(E, p_{j} \hat{L}\right) \phi_{D, j}\left(E, p_{j} \hat{L}\right) 2 E e^{-i E t+i p_{j} L}
$$

\footnotetext{
${ }^{8}$ We will consider that the flux is normalized as if the beam is composed of just one particle.

${ }^{9}$ The differential probability is, as the differential cross-section, a differential quantity in kinematical variables.
} 
with $\hat{L}=\frac{\overrightarrow{\mathrm{L}}}{L}$ and $p_{j}=\sqrt{E^{2}-m_{j}^{2}}$. The total probability is given by the square of this amplitude

$$
\begin{aligned}
P_{\alpha \beta}^{\mathrm{tot}}(T, \mathrm{~L}) & =\left|\mathcal{A}_{\alpha \beta}\right|^{2}=\left(\frac{1}{\left(8 \pi^{2} L\right)^{2}}\right) \sum_{j, k} U_{\alpha j}^{*} U_{\beta j} U_{\alpha k} U_{\beta k}^{*} \\
& \times \int d E \int d E^{\prime} \phi_{P, j}\left(E, p_{j} \hat{L}\right) \phi_{D, j}\left(E, p_{j} \hat{L}\right)(2 E) \\
& \times \phi_{P, k}^{*}\left(E^{\prime}, p_{k} \hat{L}\right) \phi_{D, k}^{*}\left(E^{\prime}, p_{k} \hat{L}\right)\left(2 E^{\prime}\right) e^{-i\left(E-E^{\prime}\right) t+i\left(p_{j}-p_{k}\right) L} .
\end{aligned}
$$

Assuming the production and detection processes to be isotropic we can average over the directions and drop the dependence on $\hat{L}$ by defining $\phi_{P, D}\left(E, p_{j}\right) \equiv \int \frac{d \Omega}{(4 \pi)} \phi_{D, P}\left(E, p_{j} \hat{L}\right)$. In addition to the result above we will also need the production and detection probabilities

$$
\begin{aligned}
P_{\alpha}^{\text {prod }} & =\sum_{j}\left|U_{\alpha j}\right|^{2} \frac{1}{8 \pi^{2}} \int d E\left|\phi_{P, j}\left(E, p_{j}\right)\right|^{2} 4 E p_{j}, \\
P_{\beta}^{\text {det }} & =\sum_{k}\left|U_{\beta k}\right|^{2}\left|\phi_{D, k}\left(E, p_{k}\right)\right|^{2} \frac{1}{V},
\end{aligned}
$$

where $V$ is the normalization volume. In a real experiment running for a macroscopic time $T_{0}$, bigger than any characteristic time of the processes, we have $N_{P}$ particles entering the production region ${ }^{10}$ during $T_{0}$. So the number of particles entering the region during the interval $d t_{P}$ is given by

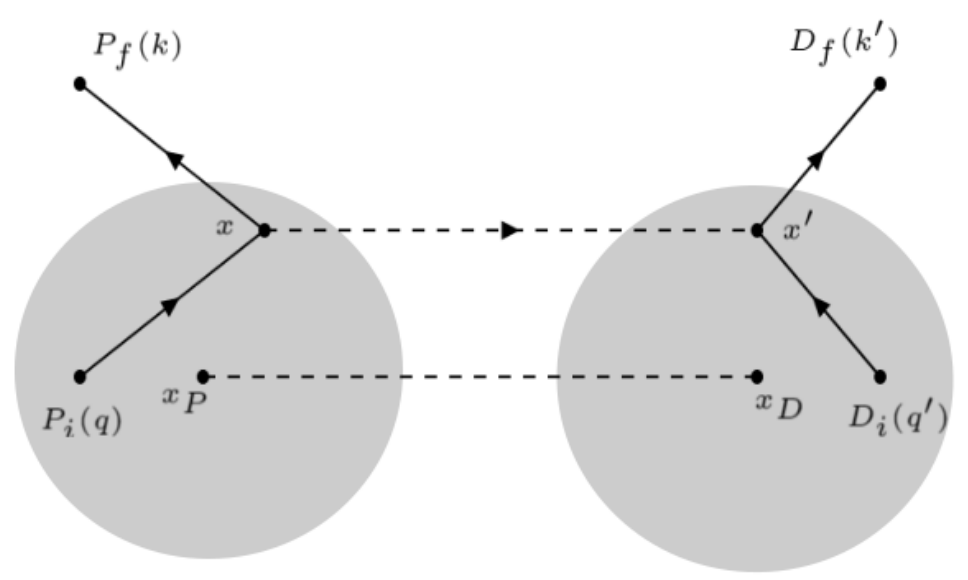

Figure 1.2: Representation of the processes happening at the production and detection regions.

\footnotetext{
${ }^{10}$ Here we will assume the flux to be steady, in references $[49,45,60]$ the authors studied a different hypothesis.
} 


$$
d N_{P}=N_{P} \frac{d t_{P}}{T_{0}} .
$$

Then the probability of neutrino emission during the time interval $t$ is

$$
\mathcal{P}_{\alpha}^{\text {prod }}(t)=N_{P} \int_{0}^{t} \frac{d t_{P}}{T_{0}} P_{\alpha}^{\text {prod }} .
$$

The production probability (1.28) is time-independent, therefore the integral is trivial. The production rate can then be easily found to be

$$
\Gamma_{\alpha}^{\text {prod }}=\frac{d \mathcal{P}_{\alpha}^{\text {prod }}(t)}{d t}=N_{P} \frac{P_{\alpha}^{\text {prod }}}{T_{0}}
$$

The result for the detection rate is analogously

$$
\Gamma_{\beta}^{\text {det }}=\frac{d \mathcal{P}_{\beta}^{\text {det }}(t)}{d t}=N_{D} \frac{P_{\beta}^{\text {prod }}}{T_{0}} .
$$

To compute (1.25) we must find the cross section (1.23), which is given by the rate (1.33) divided by the flux

$$
\Phi=n_{\nu_{k}} v_{\nu_{k}}=\frac{1}{V} \frac{p_{k}}{E}
$$

so that we have

$$
\sigma_{\beta}(E)=\frac{N_{D}}{T_{0}} \sum_{k}\left|U_{\beta k}\right|^{2}\left|\phi_{D, k}\left(E, p_{k}\right)\right|^{2} \frac{E}{p_{k}} .
$$

The total probability (1.27) should also be integrated over production $d t_{P}$ and detection $d t_{P}$ time intervals and multiplied by the number of particles entering each region during the time $T_{0}$. Performing a clever change of variables and considering the time interval bigger than any characteristic time scale of any of the processes it is possible to show that the two integrals are equivalent to integrate (1.27) over time $T$ [44]. The integration results in a delta function assuring energy conservation and then including the multiplicative factors we have the result for the overall production rate

$$
\Gamma_{\alpha \beta}^{\mathrm{tot}}(L)=N_{P} N_{D} \frac{P_{\alpha \beta}^{\mathrm{tot}}(L)}{T_{0}^{2}},
$$

Plugging (1.32), (1.35) and (1.36) together into (1.25) we have, 


$$
P_{\alpha \beta}(L, E)=\frac{\sum_{j, k} U_{\alpha j}^{*} U_{\beta j} U_{\alpha k} U_{\beta k}^{*} \phi_{P, j}\left(E, p_{j}\right) \phi_{D, j}\left(E, p_{j}\right) \phi_{P, k}^{*}\left(E, p_{k}\right) \phi_{D, k}^{*}\left(E, p_{k}\right) e^{i\left(p_{j}-p_{k}\right) L}}{\sum_{j}\left|U_{\alpha j}\right|^{2}\left|\phi_{P, j}\left(E, p_{j}\right)\right|^{2} p_{j} \sum_{k}\left|U_{\beta k}\right|^{2}\left|\phi_{D, k}\left(E, p_{k}\right)\right|^{2} p_{k}^{-1}} .
$$

Now the Quantum Field theoretical approach allows us to see under which conditions the expression (1.37) can be interpreted as a real probability. Recall that the dependence of $\phi$ on the indexes $j(k)$ are due to the neutrino momentum $p_{j}\left(p_{k}\right)$. The convolution of wave packets will result in another wave packet peaked at mean momentum $\overrightarrow{\mathrm{P}}^{\prime}$, hence if $\left|p_{j}-p_{k}\right|$ is smaller than the wave packet width $\sigma_{p P}$ we are allowed to replace all the $p_{j}$ by a common value $p$ given by the average over the different neutrino mass eigenstates momentum. This condition also implies that we can replace the factors $p_{k}^{-1}$ and $p_{j}$ by $p$ [44]. Finally, we have

$$
P_{\alpha \beta}(L, E)=\sum_{j, k} U_{\alpha j}^{*} U_{\beta j} U_{\alpha k} U_{\beta k}^{*} e^{i\left(p_{j}-p_{k}\right) L} .
$$

Under the conditions above, this framework gives the probabilities used in the study of neutrino oscillations properly normalized. This is much more reliable in comparison with the plane wave description since the assumptions are restricted to properties of the neutrinos, no equal energy or equal momentum hypothesis was made.

\subsubsection{Additional remarks}

Here we will discuss further details and make considerations about the external wave packet approach. First of all, if we used plane waves in equation (1.22) instead of wave packets, we would obtain,

$$
\phi_{P, j}=(2 \pi)^{4} \delta^{4}(p-Q-K) \frac{M_{P, j}(Q, K)}{\sqrt{2 E_{P, i}(Q) V 2 E_{P, f}(K) V}} .
$$

In this case, the phase of the amplitude is $e^{-i(Q-K) L}$, which does not depend on the mass of the intermediate state, so oscillations would not happen.

In appendix A we expanded the energy only up to first order, this was enough for our present goal in this chapter. But the second-order expansion allows us to study the dispersion of the wave packet in detail

$$
E_{i}(p) \approx E(P)+\overrightarrow{\mathrm{v}}_{i} \cdot(\overrightarrow{\mathrm{p}}-\overrightarrow{\mathrm{P}})+\frac{1}{2 E(P)}\left((\overrightarrow{\mathrm{p}}-\overrightarrow{\mathrm{P}})^{2}-\left(\overrightarrow{\mathrm{v}_{\mathrm{i}}} \cdot(\overrightarrow{\mathrm{p}}-\overrightarrow{\mathrm{P}})\right)^{2}\right)
$$


where $\overrightarrow{\mathrm{v}}_{i}=\overrightarrow{\mathrm{p}} / E_{i}(\mathrm{P})$ is the velocity of the $i$ th component of the wave packet. It is straightforward to find the configuration space wave packet if we consider the wave packets to be described by a Gaussian function

$$
f_{\nu_{j}}(\overrightarrow{\mathrm{x}}, t)=\frac{\left(2 \pi \sigma_{x, i}^{2}\right)^{-3 / 4}}{\sqrt{\operatorname{det}(\Sigma)}} \exp \left(-i E_{j}(P) t+i \overrightarrow{\mathrm{P}} \cdot \overrightarrow{\mathrm{x}}-\frac{\left(\overrightarrow{\mathrm{x}}_{j}-\overrightarrow{\mathrm{v}}_{j} t\right) \Sigma^{-1}\left(\overrightarrow{\mathrm{x}}_{j}-\overrightarrow{\mathrm{v}}_{j} t\right)}{4 \sigma_{x, i}^{2}}\right)
$$

Considering the velocity, $\overrightarrow{\mathrm{v}}_{j}$ to be aligned with the $z$ axis we can easily find the spatial uncertainty

$$
\left\langle\left(\Delta x^{a}\right)^{2}\right\rangle=\sigma_{x, i}^{2} \frac{1}{\left[\operatorname{Re}\left(\Sigma^{-1}\right)\right]^{a a}} .
$$

The diagonal elements of $\operatorname{Re}\left(\Sigma^{-1}\right)^{-1}$ are

$$
\begin{aligned}
& \left(\operatorname{Re}\left(\Sigma^{-1}\right)^{-1}\right)^{x x}=\left(\operatorname{Re}\left(\Sigma^{-1}\right)^{-1}\right)^{y y}=1+\frac{4 \sigma_{p, i}^{4} t^{2}}{E_{j}^{2}(\mathrm{P})}, \\
& \left(\operatorname{Re}\left(\Sigma^{-1}\right)^{-1}\right)^{z z}=1+\frac{4 m_{j}^{4} \sigma_{x, i}^{4} t^{2}}{E_{j}^{6}(\mathrm{P})} .
\end{aligned}
$$

Those equations show that the wave packet spreading is anisotropic, we have three regimes

(a) No dispersion regime, before $L \leq \frac{p}{\sigma_{p}^{2}}$;

(b) Disperson in the transversal direction if $\frac{p}{\sigma_{p}^{2}} \leq L \leq \frac{p E}{2 m_{j}^{2} \sigma_{p}^{2}}$;

(c) Disperson in all directions at $L>\frac{p E}{2 m_{j}^{2} \sigma_{p}^{2}}$;

the Grimus-Stockinger theorem [65] is valid if the condition $L \gg p_{j} / \sigma_{p}^{2}$ is satisfied [49]. For atmospheric neutrinos considering $p=1 \mathrm{GeV}$ and that the momentum uncertainty is $\sigma_{p}=$ $10^{-6} \mathrm{GeV}$ we can estimate $p / \sigma_{p}^{2} \approx 10^{-7} \mathrm{~km}$, also under those conditions the dispersion length is of order $10^{5} \mathrm{~km}[45,66]$, hence we can safely say that we are in the second regime. Furthermore, the coherence length can also be estimated $L^{\mathrm{coh}} \approx 10^{9} \mathrm{~km}$ which is much bigger than the Earth diameter and then, for experiments searching for atmospheric neutrinos or produced by any source on Earth, we can safely neglect the two newer terms in (1.12).

In summary, while the naive plane-wave method is theoretically and physically inconsistent the final result gives the same answer if all those conditions are satisfied. The oscillation phenomenon is so special, in the sense that small differences would completely erase the pattern, 
that we can consider those particles as one of the most fundamental quantum systems we have ever dealt with. Neutrinos from different sources reach the Earth and not all might satisfy all the conditions to neglect the new terms, therefore further tests of Quantum Mechanics could then be performed through the oscillation phenomena and maybe there are interesting new insights about the world waiting to be discovered in there.

\subsection{Matter effects}

The last section was devoted to the study of the underlying details of the oscillation phenomena, the complications associated with considering matter effects would only make the discussion harder, without changing the conclusion [48]. But neutrinos will unavoidable travel through a medium while propagating, in particular we want to study neutrinos that travel through the Earth before being detected. Therefore, the changes due to the interactions with the particles in matter cannot be neglected. The process is characterized by coherent forward scattering of neutrinos on background fermions, it will introduce an extra term on the vacuum Hamiltonian, changing the oscillation pattern. Two different interactions might happen, one described by the charged current Lagrangian

$$
\mathcal{L}_{C C}=-\frac{G_{F}}{\sqrt{2}}\left(\bar{\nu}_{e}(x) \gamma^{\mu}\left(1-\gamma_{5}\right) e(x)\right)\left(\bar{e}(x) \gamma_{\mu}\left(1-\gamma_{5}\right) \nu_{e}(x)\right),
$$

where $\nu_{e}(x)$ and $e(x)$ are the electron neutrino and electron fields respectively, and $G_{F}$ the Fermi coupling constant. The other interaction is given by the neutral current Lagrangian

$$
\mathcal{L}_{N C}=-\frac{G_{F}}{\sqrt{2}}\left(\bar{\nu}_{\alpha}(x) \gamma^{\mu}\left(1-\gamma_{5}\right) \nu_{\alpha}(x)\right)\left(\bar{\psi}_{f}(x) \gamma_{\mu}\left(T_{3 L f}-2 Q_{f} \sin ^{2} \theta_{W}\right) \psi_{f}(x)\right)
$$

where $\nu_{\alpha}(x)$ and $\psi_{f}(x)$ are the fields representing the $\alpha$ flavored neutrino $(\alpha=e, \mu, \tau)$ and the field of a fermion $f=e, p, n$, respectively. The factor $Q_{f}$ is the electric charge of fermion $f$, $T_{3 L f}$ is the third component of the isospin operator associated with the left handed fermion $f$ and $\theta_{W}$ is the Weinberg angle.

Note that we have included in (1.45) only the electron neutrino, this is justified because we are considering usual matter with no muons or taus present. Assuming the matter to be unpolarized and to have an equal number of protons and electrons, it is possible to show that the resultant effect is to add a potential $V$ to the vacuum Hamiltonian ${ }^{11}$ (1.2), in the mass basis we have

\footnotetext{
${ }^{11}$ For details see $[48,67]$.
} 


$$
H_{M}=H+U^{\dagger} V U
$$

where

$$
V= \pm \sqrt{2} G_{F}\left(\begin{array}{ccc}
n_{e}-\frac{n_{n}}{2} & & \\
& -\frac{n_{n}}{2} & \\
& & -\frac{n_{n}}{2}
\end{array}\right)
$$

with $n_{i}(i=n, e)$, is the particle number density in the medium and the plus sign + is for neutrinos and the minus sign - for antineutrinos. The matrix $U$ is the PMNS matrix in three generations, parametrized as

$$
U=U_{23} U_{13} U_{12} U_{\mathrm{Maj}}
$$

where

$U_{23}=\left(\begin{array}{ccc}1 & 0 & 0 \\ 0 & c_{23} & s_{23} \\ 0 & -s_{23} & c_{23}\end{array}\right), \quad U_{13}=\left(\begin{array}{ccc}c_{13} & 0 & s_{13} e^{-i \delta_{\mathrm{CP}}} \\ 0 & 1 & 0 \\ -s_{13} e^{i \delta_{\mathrm{CP}}} & 0 & c_{13}\end{array}\right), \quad U_{12}=\left(\begin{array}{ccc}c_{12} & s_{12} & 0 \\ -s_{12} & c_{12} & 0 \\ 0 & 0 & 1\end{array}\right)$,

and

$$
U_{\text {Maj }}=\left(\begin{array}{ccc}
1 & 0 & 0 \\
0 & e^{i \phi_{1}} & 0 \\
0 & 0 & e^{i \phi_{2}}
\end{array}\right)
$$

We adopted the shorthand notation $c_{i j}\left(s_{i j}\right)=\cos \theta_{i j}\left(\sin \theta_{i j}\right)$. Since modifications proportional to the identity matrix do not affect the oscillation probabilities only the electron number density is relevant. The matter potential became famous when physicists were struggling to solve the solar neutrino problem [9]. It is possible to show that the matter potential introduces a resonance peak on the oscillation probability, which enhances the flavor changing oscillations resulting in a deficit of observed neutrino events in experiments designed to detect only one flavor of neutrinos, this is the Mikheev-Smirnov-Wolfenstein (MSW) effect [68]. 


\subsection{Conclusions}

In summary, we have studied neutrino oscillation probabilities in different frameworks, the most robust being the Quantum Field Theoretical description. This was useful to see the flaws and improvements of each description. Also, we discussed how matter effects are implemented in the oscillation phenomena which will be important in the following chapters. By now we hope to have made clear how the oscillation phenomena carry rich physical insights and how changes in the predictions can be a signal of new physics or even flaws in the most fundamental theories we have so far. This fact has motivated many authors to consider new effects that might affect the oscillation pattern, for a few examples see [69, 70, 71].

In this dissertation, we want to discuss a different kind of effect, the Open Quantum System effects, following a phenomenological approach. This effect arises when the neutrino system is considered as a subsystem of a larger system which can be something that we are not even aware of. Quantum gravity, for example, might violate quantum mechanics [72], acting like an intrinsic external agent that we can never get rid of. The Open Quantum System framework is useful because it takes into account our ignorance about what might be influencing neutrino evolution, and also allows us to derive measurable consequences through the predictions for oscillations. To study this scenario the Open Quantum System formalism must be introduced. This is the subject of the next chapter. 


\section{CHAPTER 2}

\section{THE FORMALISM OF OPEN QUANTUM SYSTEMS}

“...quantum phenomena do not occur in a Hilbert space, they occur in a laboratory."

(Asher Peres)

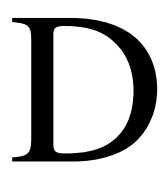

URING our first contact with Quantum Mechanics, we must learn the necessary mathematical tools to understand the theory. Concepts such as Hilbert spaces $\mathcal{H}$ and important mathematical relations, for example, completeness relations, inner products, and normalization of states are introduced. After that the foundation of Quantum Mechanics is established by a set of postulates:

(i) The state of a physical system at a fixed time $t_{0}$ is fully determined by a vector of the Hilbert space $\mathcal{H}$ which is called a ket and denoted by $\left|\psi\left(t_{0}\right)\right\rangle$.

(ii) Measurable physical quantities are described by Hermitian operators acting on $\mathcal{H}$, this subset of operators of the space are called observables.

(iii) The possible outcomes of an observable are its eigenvalues

(iv) Let $\mathcal{O}$ be an observable with eigenvalues described by the set $\left\{o_{n}\right\}$ and eigenvectors $\left\{\left|O_{n}\right\rangle\right\}$. The probability of obtaining the value $o_{n}$ when measuring $\mathcal{O}$ in the state $|\psi\rangle$ is ${ }^{1}$

$$
\mathcal{P}\left(o_{n}\right)=\left|\left\langle O_{n} \mid \psi\right\rangle\right|^{2},
$$

\footnotetext{
${ }^{1}$ We are considering here a discrete, non-degenerate spectrum, the generalization is straightforward.
} 
after the measurement, the state is described by the normalized projection

$$
\frac{P_{n}|\psi\rangle}{\sqrt{\left\langle\psi\left|P_{n}\right| \psi\right\rangle}}
$$

where $P_{n}$ is the projector on the $n t h$ eigenstate of the observable. This process is called the collapse of the wave function.

(v) The time evolution of the state vector is dictated by the Schrödinger equation

$$
i \frac{d}{d t}|\psi(t)\rangle=H(t)|\psi(t)\rangle
$$

where $H(t)$ is the Hamiltonian operator of the system. For future purposes we considered it to be time dependent.

Besides the astonishing success the theory has achieved with predictions based on the above set of rules, it is important to discuss its limitations. First of all, the postulates, as enunciated, describe a closed system which is a physical system that is not interacting with its surroundings either by exchanging matter or energy. In a real situation, this could never be true, as there is always an external agent interacting with the system. This is not a matter of improving experimental conditions, even though we conduct an experiment in a complete vacuum we cannot get rid of quantum fluctuations. When the effects of such agents cannot be neglected, there are two options to follow. One is to redefine the system to incorporate the external agent and the other is to develop an Open Quantum System formalism. This second option happens to be the case when we have no certainty regarding what can interfere with our system and it can also be interesting to provide further tests for the foundations of the quantum theory.

Another interesting point related to the discussion above is motivated by postulate (iv), which is one of the peculiar aspects of the theory. In an Open Quantum System framework, it can be recast in a more acceptable formalism, yet not a fully satisfying one. Also, the discussions about how to interpret Quantum Mechanics require an Open Quantum System approach. Following the standard Copenhagen interpretation, there is a clear separation between the system being measured and the measuring apparatus, that acts as an external agent. Other proposals, such as the Everett or "multiverse" interpretation [73], consider that any system is a composite system of all possible states in the universe and the experiment by definition will, as a result, always deal with an Open System. To move from the notion of a state as defined in (i) to a broader framework, suited to study composite systems, entanglement, and to analyze Open Systems we need first to introduce the necessary tools. In this chapter, we will define the density operator, 
which is the extension of the previous definition of a state, analyze its properties, normalization, and time evolution. Then using this operator, we will study the important operations in composite systems and their classifications. Finally, we will derive the time evolution for an Open Quantum System and exhibit quantifiers that are useful tools to study new effects.

\subsection{Density Operators}

An operator $\rho$ is considered a valid density operator if the following properties are satisfied:

(1) $\langle\psi|\rho| \psi\rangle \geq 0$, for any state $|\psi\rangle$;

(2) $\operatorname{Tr}\{\rho\}=1$;

(3) $\rho=\rho^{\dagger}$.

To illustrate the necessity of this operator without recurring to abstract arguments, consider that we want to study a physical state $|\psi\rangle$ but our experimental setup is not perfect and creates also the state $|\beta\rangle$ with probability $p$. So we will actually produce the superposition of states $|\chi\rangle$ given by

$$
|\chi\rangle=(1-p)|\psi\rangle+p|\beta\rangle
$$

The crucial point here is the mixture between a classical uncertainty (the fact that our setup is not ideal) and the quantum states. This is already something the previous definition cannot deal with. Requiring that the density operator carries both the information about all the possible outcomes of the experiment and the probability of producing each state, we can find how it looks like through the expected value of an operator $A$ in the state (2.4)

$$
\begin{aligned}
\langle\chi|A| \chi\rangle & =p\langle\beta|A| \beta\rangle+(1-p)\langle\psi|A| \psi\rangle=\sum_{i=\beta, \psi} p_{i}\langle i|A| i\rangle \\
& =\operatorname{Tr}\left\{A \sum_{i=\beta, \psi} p_{i}|i\rangle\langle i|\right\} \equiv \operatorname{Tr}\{A \rho\} .
\end{aligned}
$$

The extension to the case where $\mathrm{n}$ states can be produced is $\rho=\sum_{i=1}^{n} p_{i}|i\rangle\langle i|$, with $p_{i}$ being the probabilities of producing the $i t h$ state. It is effortless to demonstrate that this operator satisfies all the required properties and is a valid density operator. Due to the normalization condition (2) the coefficients $p_{i}$, even in complicated situations, will always sum up to one and 
then be interpreted as probabilities ${ }^{2}$. One general important feature of the density operator is the fact that the trace of its square is always less or equal one, which can be seen in the last example (see figure 2.1). In that case, $\operatorname{Tr}\left\{\rho^{2}\right\}=1$ only if either $p=0$ or $p=1$. This fact motivates the separation between states saturating the equality, called pure states, and the others which are denominated mixed states.

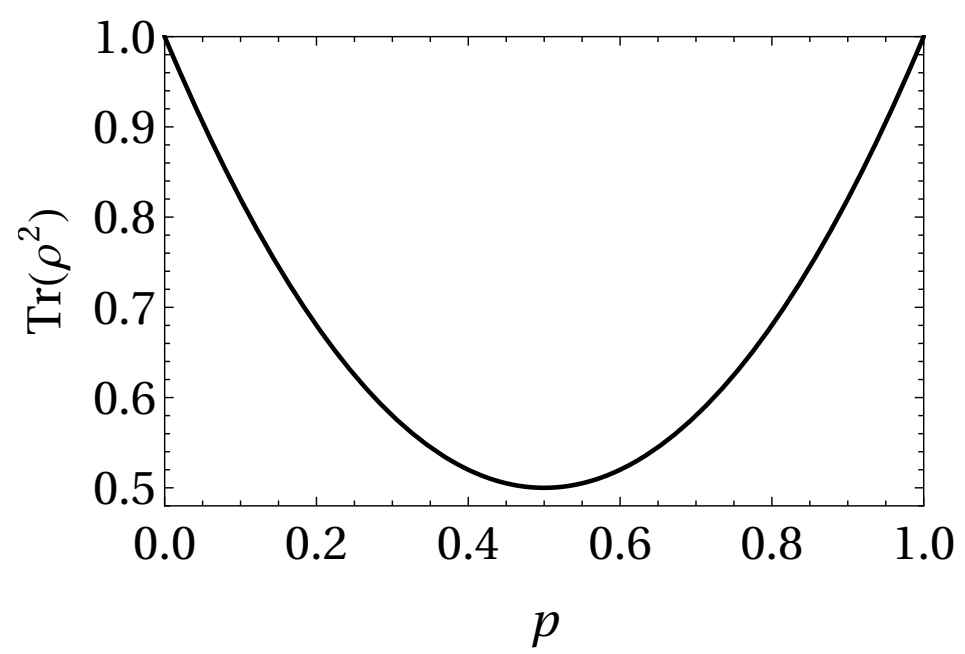

Figure 2.1: The behaviour of $\operatorname{Tr}\left\{\rho^{2}\right\}$ considering the example (2.4)

If the Hilbert space under study is finite-dimensional, there also exists a lower bound for the value of $\operatorname{Tr}\left\{\rho^{2}\right\}$, which is equal to $\frac{1}{N}$, where $N$ is the dimension of $\mathcal{H}$ corresponding to the case in which all the possible states are equally probable

$$
\frac{1}{N} \leq \operatorname{Tr}\left\{\rho^{2}\right\} \leq 1
$$

\subsubsection{Time Evolution}

The time evolution of the density operator is inherited from (2.3)

$$
\begin{aligned}
\frac{d \rho(t)}{d t} & =\sum_{j} p_{j}\left(\left(\frac{d}{d t}|\psi\rangle\right)\langle\psi|+| \psi\rangle\left(\frac{d}{d t}\langle\psi|\right)\right) \\
& =-i[H(t), \rho(t)] .
\end{aligned}
$$

The above equation is known as the von Neumann equation. For future purposes, it is useful to recast the time evolution in the following form

\footnotetext{
${ }^{2}$ From now on we shall call both $\rho$ and the vectors $|\psi\rangle$ a state.
} 


$$
\frac{d \rho(t)}{d t}=\mathcal{L}(t) \rho(t) \equiv-i[H(t), \rho(t)]
$$

where $\mathcal{L}(t)$ is called a super operator ${ }^{3}$ whose action on the density operator dictates the time evolution. The evolved state can then be formally written as

$$
\rho(t)=\mathrm{T}\left\{\exp \left[\int_{t_{0}}^{t} \mathrm{~d} t^{\prime} \mathcal{L}\left(t^{\prime}\right)\right]\right\} \rho\left(t_{0}\right),
$$

with $\mathrm{T}\{\}$ being the time ordering operator. The previous equation is still valid only for closed systems, we shall see how to extend the formalism to describe open systems.

\subsubsection{Composite systems and the partial Trace}

At the moment we have only analyzed systems consisting of a single Hilbert space. Our future goal is to study the consequences of considering neutrinos as a subsystem immersed in a larger system and for this purpose we must go beyond the single system description. Suppose that instead of only one system characterized by $\mathcal{H}_{1}$ and the Hamiltonian $H_{1}$, we also have a copy of the system characterized by $\left(\mathcal{H}_{2}, H_{2}\right)$. The full Hilbert space is given by the tensor product $\mathcal{H}_{T}=\mathcal{H}_{1} \otimes \mathcal{H}_{2}$, if the state of each system is known we can define the total state to be $\rho_{T}=\rho_{1} \otimes \rho_{2}$. However, the converse is not true, if $\rho_{T}$ is given we cannot write it as $\rho_{1} \otimes \rho_{2}$ in general. Only when both states are uncorrelated we can write them in this form, called the product state decomposition.

Quantum correlated, or entangled, states have generated a lot of discussion about the foundations of Quantum Mechanics, the most famous being the EPR paradox [74]. The way to classify a state concerning its correlations is by studying the partial trace of the complete state. The operation consists of summing over all degrees of freedom associated with the system we want to exclude. This may be useful, for instance, when the full dynamic is intricate. If $\rho_{1}=\left|\psi_{1}\right\rangle\left\langle\psi_{1}\right|$ and $\rho_{2}=\left|\psi_{2}\right\rangle\left\langle\psi_{2}\right|$, then $\rho_{T}=\left|\psi_{1}\right\rangle\left\langle\psi_{1}|\otimes| \psi_{2}\right\rangle\left\langle\psi_{2}\right|$. We can trace over the second state obtaining

$$
\operatorname{Tr}_{2} \rho_{T}=\left|\psi_{1}\right\rangle\left\langle\psi_{1}\right| \delta_{22^{\prime}}
$$

After performing the partial trace we are left with the reduced dynamics, we can then define the partial states through

\footnotetext{
${ }^{3}$ The nomenclature is because $\mathcal{L}(t)$ is an operator acting on another operator.
} 


$$
\begin{aligned}
& \rho_{1}=\operatorname{Tr}_{2}\left\{\rho_{T}\right\}, \\
& \rho_{2}=\operatorname{Tr}_{1}\left\{\rho_{T}\right\} .
\end{aligned}
$$

Note that the remaining operator acts only on the remaining Hilbert space. Consider now $\mathcal{O}_{1} \otimes \mathcal{I}_{2}$, an operator acting non trivially only on $\mathcal{H}_{1}$, its expected value is given by

$$
\left\langle\mathcal{O}_{1} \otimes \mathcal{I}_{2}\right\rangle=\operatorname{Tr}\left\{\mathcal{O}_{1} \otimes \mathcal{I}_{2} \rho_{T}\right\}=\operatorname{Tr}_{1}\left\{\mathcal{O}_{1} \rho_{1}\right\}
$$

This is the defining property of the operation. One important point to keep in mind is the fact that the partial trace is an irreversible operation so, in general, it is not possible to recover all the information about $\rho_{T}$, the only exception being the situation where the complete state is a product state. The reduced state can be classified by its purity and correlations, for a complete list considering all the possible cases see [75]. At this point, for future purposes, it is interesting to introduce some useful terminology.

Consider the quantum superposition $|\psi\rangle=\alpha\left|\phi_{1}\right\rangle+\beta\left|\phi_{2}\right\rangle$, the density operator associated is

$$
\rho=\left(\begin{array}{cc}
|\alpha|^{2} & \alpha \beta^{*} \\
\alpha^{*} \beta & |\beta|^{2}
\end{array}\right) .
$$

The diagonal terms are called populations and the off-diagonal terms are called coherence terms. This terminology can be easily extended to higher dimensions.

As we shall see the equation governing the evolution of Open Quantum Systems is not unitary, being able to change the purity of a state by destroying the coherence terms and changing the populations. The concept of coherence is clearly basis dependent. The interactions of the subsystem with the larger system select a preferred basis.

\subsection{Open Quantum Systems}

Hitherto we have reviewed all the interesting points about closed quantum systems, now let us turn to Open Quantum Systems. We call a quantum system $S$ open if it is coupled to another quantum system $E$, usually called the Environment. The composite system $S+E$ is assumed to obey the usual unitary evolution, while the reduced state in $S$ will change as a consequence of its internal dynamics and also from its interactions with $E$. The correlations which might arise from such interactions make the reduced evolution nonunitary. 
Let $\mathcal{H}_{S}, \mathcal{H}_{E}$ and $\mathcal{H}_{S} \otimes \mathcal{H}_{E}$ be the Hilbert spaces associated with $S, E$ and $S+E$ respectively. We can write the most general Hamiltonian as

$$
H(t)=H_{S}(t) \otimes \mathcal{I}_{E}+\mathcal{I}_{S} \otimes H_{E}(t)+H_{I}(t)
$$

where $H_{S}(t)$ and $H_{E}(t)$ are the Hamiltonians solely of the system $S$ and $E$ respectively, and $H_{I}(t)$ describes the interactions between both systems. Let $\rho_{S+E}$ be the state of the whole system, since the evolution of the complete state is unitary we have

$$
\rho_{S+E}(t)=U\left(t, t_{0}\right) \rho_{S+E}\left(t_{0}\right) U^{\dagger}\left(t, t_{0}\right) .
$$

The reduced evolution of the subsystem $S$ can then be found by tracing over the degrees of freedom associated with the Environment

$$
\rho_{S}(t)=\operatorname{Tr}_{E}\left\{U\left(t, t_{0}\right) \rho_{S+E}\left(t_{0}\right) U^{\dagger}\left(t, t_{0}\right)\right\}
$$

Recall that from (2.7) we can also find the evolution through

$$
\begin{aligned}
\operatorname{Tr}_{E}\left\{\frac{d \rho(t)}{d t}\right\} & =-i \operatorname{Tr}_{E}\{[H(t), \rho(t)]\} \\
\frac{d \rho_{S}(t)}{d t} & =-i \operatorname{Tr}_{E}\{[H(t), \rho(t)]\}
\end{aligned}
$$

The differential equation above can be very difficult to solve and, perhaps, neither the environment nor its dynamics is well known. This motivates a general description concerning only the reduced state $\rho_{S}$.

\subsubsection{Dynamical maps}

A map $V\left(t, t_{0}\right)$ responsible for the evolution of the state of the subsystem at time $t_{0}$ to the instant $t$ is called a dynamical map

$$
V\left(t, t_{0}\right): \rho_{S}\left(t_{0}\right) \mapsto \rho_{S}(t)
$$

Usually $V\left(t, t_{0}\right)$ depends on the state $\rho_{S}\left(t_{0}\right)$ due to the correlations acquired from the interactions with the Environment. A dynamical map which is independent of the state is called a Universal Dynamical Map (UDM). A sufficient and necessary condition for a dynamical map to be universal is that the initial state of the whole system, from which the operator will be 
inherited, needs to be a product state $\rho_{S+E}\left(t_{0}\right)=\rho_{S}\left(t_{0}\right) \otimes \rho_{E}\left(t_{0}\right)$, where $\rho_{E}\left(t_{0}\right)$ is fixed for all $\rho_{S}\left(t_{0}\right)$ (see reference [76]).

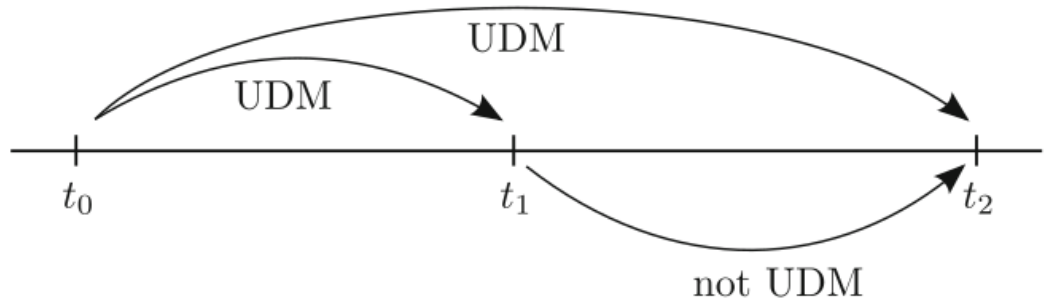

Figure 2.2: A necessary and sufficient condition for a map to be universal is that the initial state of the whole system must be written as a product state. On the other hand, the evolved state, in general, cannot be written in this form. Therefore, there is no guarantee that the inherited map will be a Universal Dynamical Map. Figure taken from [76].

This is very important, considering that we start with $\rho_{S+E}\left(t_{0}\right)$ given as a product of states, we can evolve the reduced state $\rho_{S}\left(t_{0}\right)$ by using a UDM to any future instant of time. However, nothing guarantees that if, for instance, we evolve the system to $t_{1}$ and then to $t_{2}>t_{1}$, the second step is going to be a UDM, since the state at $t_{1}$ usually cannot be written as a product of states turning the inherited map state-dependent. Therefore, the composition rule

$$
U\left(t_{2}, t_{0}\right)=U\left(t_{2}, t_{1}\right) U\left(t_{1}, t_{0}\right)
$$

does not hold for all Universal Dynamical Maps.

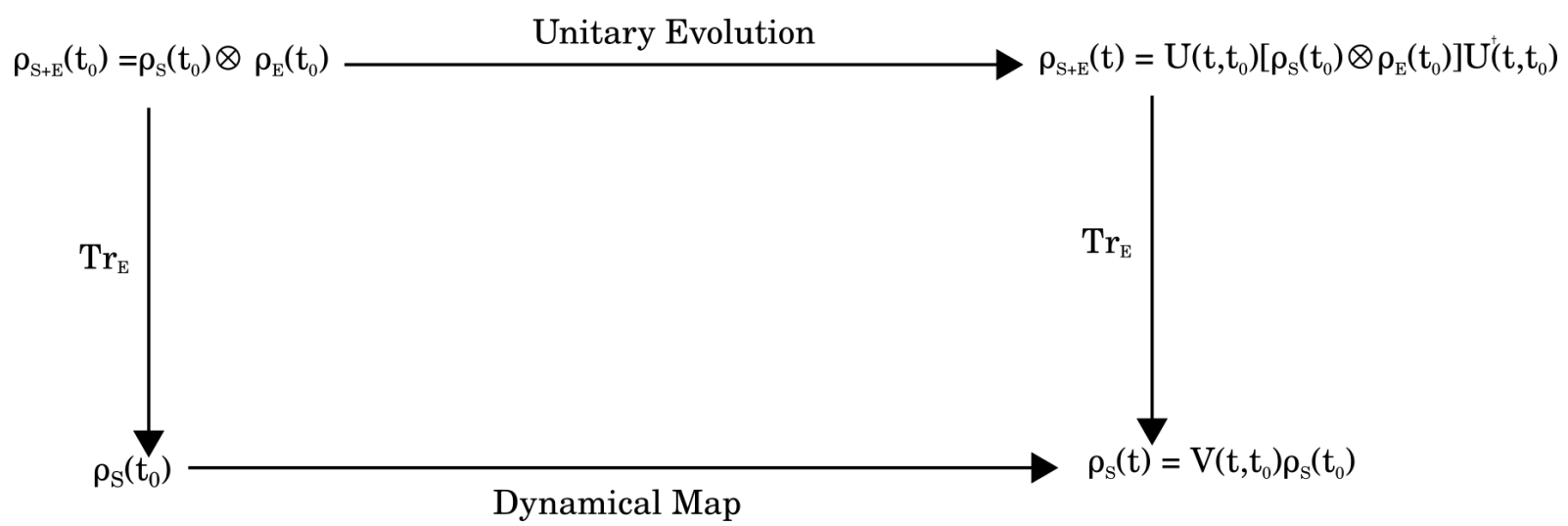

Figure 2.3: Summary of the evolution of the reduced state $\rho_{S}$. 


\subsubsection{Markovian processes and the Quantum Master Equation}

If the correlations the subsystem develops do not affect much the dynamics of $S$ we recover the property (2.19). This happens to be the case, for example, when the correlation decays faster than the characteristic time of the process under study occurring in the subsystem. Under this assumption memoir effects can be neglected and we recover the composition rule such that the Universal Dynamical Maps $\left\{V\left(t, t_{0}\right), t \geq t_{0}\right\}$ form a two-parameter family obeying the same structure of a semigroup, with $V\left(t_{0}, t_{0}\right)$ being the identity ${ }^{4}$. In this regime, we can derive a differential equation for the time evolution of the reduced state [77].

The time evolution of $S$ is given by (see figure 2.3)

$$
\rho_{S}(t)=V\left(t, t_{0}\right) \rho_{S}\left(t_{0}\right) \equiv \operatorname{Tr}_{E}\left\{U\left(t, t_{0}\right)\left[\rho_{S}\left(t_{0}\right) \otimes \rho_{E}\left(t_{0}\right)\right] U^{\dagger}\left(t, t_{0}\right)\right\}
$$

If we consider the spectral decomposition of the Environment

$$
\rho_{E}\left(t_{0}\right)=\sum_{\beta} \lambda_{\beta}\left|\phi_{\beta}\left(t_{0}\right)\right\rangle\left\langle\phi_{\beta}\left(t_{0}\right)\right|
$$

where $\left|\phi_{\beta}\right\rangle$ form an orthonormal set of vectors in $\mathcal{H}_{E}$ and $\sum_{\alpha} \lambda_{\alpha}=1$, equation (2.20) results in

$$
\begin{aligned}
V\left(t, t_{0}\right) \rho_{S}\left(t_{0}\right) & =\operatorname{Tr}_{E}\left\{U\left(t, t_{0}\right)\left[\rho_{S}\left(t_{0}\right) \otimes \sum_{\beta} \lambda_{\beta}\left|\phi_{\beta}\left(t_{0}\right)\right\rangle\left\langle\phi_{\beta}\left(t_{0}\right)\right|\right] U^{\dagger}\left(t, t_{0}\right)\right\}, \\
& =\sum_{\alpha}\left\langle\phi_{\alpha}\left(t_{0}\right)\left|U\left(t, t_{0}\right)\left[\rho_{S}\left(t_{0}\right) \otimes \sum_{\beta} \lambda_{\beta}\left|\phi_{\beta}\left(t_{0}\right)\right\rangle\left\langle\phi_{\beta}\left(t_{0}\right)\right|\right] U^{\dagger}\left(t, t_{0}\right)\right| \phi_{\alpha}\left(t_{0}\right)\right\rangle, \\
& =\sum_{\alpha, \beta} \sqrt{\lambda_{\beta}}\left\langle\phi_{\alpha}\left(t_{0}\right)\left|U\left(t, t_{0}\right)\right| \phi_{\beta}\left(t_{0}\right)\right\rangle \rho_{S}\left(t_{0}\right) \sqrt{\lambda_{\beta}}\left\langle\phi_{\beta}\left(t_{0}\right)\left|U^{\dagger}\left(t, t_{0}\right)\right| \phi_{\alpha}\left(t_{0}\right)\right\rangle, \\
& \equiv \sum_{\alpha, \beta} W_{\alpha \beta}\left(t, t_{0}\right) \rho_{S}\left(t_{0}\right) W_{\alpha \beta}^{\dagger}\left(t, t_{0}\right) .
\end{aligned}
$$

Using the completeness relation and the fact that the $\lambda_{\alpha}$ sum up to one we can show that

$$
\sum_{\alpha, \beta} W_{\alpha \beta}^{\dagger}\left(t, t_{0}\right) W_{\alpha \beta}\left(t, t_{0}\right)=\mathcal{I}_{S}
$$

Making use of the cyclic property of the trace and the above relation, we can see that the

\footnotetext{
${ }^{4}$ The family of maps, in general, does not have an inverse since the reduce dynamics is nonunitary. The only exception is a unitary map.
} 
map is trace-preserving

$$
\operatorname{Tr}_{S}\left\{V\left(t, t_{0}\right) \rho_{S}\left(t_{0}\right)\right\}=\operatorname{Tr}_{S}\left\{\rho_{S}\left(t_{0}\right)\right\}=1 \text {. }
$$

Given such a map, we want to find the associated super operator to cast the equation in the form (2.8). Assuming that $\mathcal{H}_{S}$ is a finite-dimensional Hilbert space of dimension $N$, the space of operators acting on $\rho_{S}$ has dimension $N^{2}$, using a complete basis of orthogonal operators $F_{i}$, $i=1,2, \ldots, N^{2}$, with the inner product defined as

$$
\left(F_{i}, F_{j}\right)=\operatorname{Tr}_{S}\left\{F_{i}^{\dagger} F_{j}\right\}=\delta_{i j}
$$

and choosing $F_{N^{2}}$ to be proportional to the identity, $F_{N^{2}}=\frac{1}{\sqrt{N}} \mathcal{I}_{S}$, from (2.25) the other $N^{2}-1$ operators are traceless. Expanding $W_{\alpha \beta}\left(t, t_{0}\right)$ in this basis

$$
W_{\alpha \beta}\left(t, t_{0}\right)=\sum_{i=1}^{N^{2}} F_{i}\left(F_{i}, W_{\alpha \beta}\left(t, t_{0}\right)\right)
$$

we have

$$
V\left(t, t_{0}\right) \rho_{S}\left(t_{0}\right)=\sum_{\alpha, \beta} \sum_{i=1}^{N^{2}} \sum_{j=1}^{N^{2}}\left(F_{i}, W_{\alpha \beta}\left(t, t_{0}\right)\right)\left(F_{j}, W_{\alpha \beta}\left(t, t_{0}\right)\right)^{*} F_{i} \rho_{S}\left(t_{0}\right) F_{j}^{\dagger},
$$

defining

$$
c_{i j}\left(t, t_{0}\right)=\sum_{\alpha \beta}\left(F_{i}, W_{\alpha \beta}\left(t, t_{0}\right)\right)\left(F_{j}, W_{\alpha \beta}\left(t, t_{0}\right)\right)^{*},
$$

the evolution can be written as

$$
V\left(t, t_{0}\right) \rho_{S}\left(t_{0}\right)=\sum_{i, j}^{N^{2}} c_{i j}\left(t, t_{0}\right) F_{i} \rho_{S}\left(t_{0}\right) F_{j}^{\dagger}
$$

The action of the super operator $\mathcal{L}(t)$ is then defined through the infinitesimal limit 


$$
\begin{aligned}
\mathcal{L}(t) \rho_{S}(t) & =\lim _{\epsilon \rightarrow 0} \frac{1}{\epsilon}\left(\rho_{S}(t+\epsilon)-\rho_{S}(t)\right)=\lim _{\epsilon \rightarrow 0} \frac{1}{\epsilon}\left(V(\epsilon, t) \rho_{S}(t)-\rho_{S}(t)\right) \\
& =\lim _{\epsilon \rightarrow 0}\left(\frac{1}{N} \frac{c_{N^{2} N^{2}}(\epsilon, t)-N}{\epsilon} \rho_{S}(t)+\frac{1}{\sqrt{N}} \sum_{i=1}^{N^{2}-1}\left(\frac{c_{i N^{2}}(\epsilon, t)}{\epsilon} F_{i} \rho_{S}(t)+\frac{c_{N^{2} i}(\epsilon, t)}{\epsilon} \rho_{S}(t) F_{i}^{\dagger}\right)\right. \\
& \left.+\sum_{i, j=1}^{N^{2}-1} \frac{c_{i j}(\epsilon, t)}{\epsilon} F_{i} \rho_{S}(t) F_{j}^{\dagger}\right)
\end{aligned}
$$

The second equality is easily achieved by separating the terms involving the identity $F_{N^{2}}$. Defining:

$$
\begin{array}{rlr}
a_{N^{2} N^{2}}(t) & =\lim _{\epsilon \rightarrow 0} \frac{c_{N^{2} N^{2}}(\epsilon, t)-N}{\epsilon} \\
a_{i N^{2}}(t) & =\lim _{\epsilon \rightarrow 0} \frac{c_{i N^{2}}(\epsilon, t)}{\epsilon}, & \\
a_{i j}(t) & =\lim _{\epsilon \rightarrow 0} \frac{c_{i j}(\epsilon, t)}{\epsilon}, & i, j=1, \ldots, N^{2}-1,
\end{array}
$$

and finally, introducing the operators

$$
\begin{aligned}
& G(t)=\frac{1}{2 N} a_{N^{2} N^{2}}(t) \mathcal{I}_{S}+\frac{1}{2}\left(\frac{1}{\sqrt{N}} \sum_{i=1}^{N^{2}-1} a_{i N^{2}}(t) F_{i}+\text { h.c. }\right) \\
& H(t)=\frac{1}{2 i}\left(\frac{1}{\sqrt{N}} \sum_{i=1}^{N^{2}-1} a_{i N^{2}}(t) F_{i}+\text { h.c. }\right)
\end{aligned}
$$

where h.c. stands for hermitian conjugate. The action of $\mathcal{L}(t)$ can then be written as

$$
\mathcal{L}(t) \rho_{S}(t)=-i\left[H(t), \rho_{S}(t)\right]+\left\{G(t), \rho_{S}(t)\right\}+\sum_{i, j=1}^{N^{2}-1} a_{i j}(t) F_{i} \rho_{S}(t) F_{j}^{\dagger} .
$$

Note that $H(t)$ is Hermitian. We can simplify even more the above equation by using the fact that $\mathcal{L}(t)$ is a tracing preserving operation (2.24) and making use of the cyclic property of the trace. We have that

$$
\operatorname{Tr}\left\{\mathcal{L}(t) \rho_{S}(t)\right\}=\operatorname{Tr}\left\{\left(2 G(t)+\sum_{i, j=1}^{N^{2}-1} a_{i j}(t) F_{j}^{\dagger} F_{i}\right) \rho_{S}(t)\right\}=0 .
$$


Then the action of $\mathcal{L}(t)$ on $\rho_{S}(t)$ is given by

$$
\mathcal{L}(t) \rho_{S}(t)=-i\left[H(t), \rho_{S}(t)\right]+\sum_{i, j=1}^{N^{2}-1} a_{i j}(t)\left(F_{i} \rho_{S}(t) F_{j}^{\dagger}-\frac{1}{2}\left\{F_{j}^{\dagger} F_{i}, \rho_{S}(t)\right\}\right)
$$

The above equation is the standard form of the time evolution generator. From (2.28) we can see that the matrix formed by the coefficients $c_{i j}(\epsilon, t)$ is Hermitian and positive semi-definite because, for any complex vector $\vec{u}$ the condition

$$
\sum_{i j} c_{i j}(\epsilon, t) u_{i}^{*} u_{j}=\sum_{\alpha, \beta}\left|\left(\sum u_{i} F_{i}, W_{\alpha \beta}(\epsilon, t)\right)\right|^{2} \geq 0
$$

is valid. So the matrix $a(t)$ made of the $a_{i j}(t)$ matrix elements is also a positive semi-definite matrix.

This is the most general form of a quantum time evolution generated by a UDM satisfying the semigroup property (2.19). The first efforts in order to find a description of Open Quantum Systems can be traced back to the works of Lindblad [78] and Kossakowski, Gorini and Sudarshan $[79,80]$. They considered a time independent Hamiltonian which implies that, in addition to the composition condition (2.19), the maps also satisfy the time homogeneity condition

$$
V\left(t_{2}\right) V\left(t_{1}\right)=V\left(t_{2}-t_{1}\right)
$$

such that the super operator $\mathcal{L}$ is time independent and the time ordering product in equation (2.35) is replaced by

$$
\rho(t)=e^{\mathcal{L}\left(t-t_{0}\right)} \rho\left(t_{0}\right)
$$

Due to the important contributions of both authors to the development of the Open System framework, the $\mathcal{L}$ super operator is referred to as the Lindbladian, the matrix $a$ is known as the Kossakowski matrix and equation (2.35) is commonly called as the Lindblad equation. The first term of this equation represents a unitary evolution, but $H(t)$ cannot be directly associated with the Hamiltonian of the subsystem because it may contain other terms due to the coupling with the Environment.

At this point, a few comments are necessary. First, a UDM satisfies a stronger condition than positivity, it is completely positive, which means that, if the map is extended trivially to a larger space $V\left(t, t_{0}\right) \otimes \mathcal{I}_{\kappa}$, where $\mathcal{I}_{\kappa}$ is an arbitrary space, the map is still positive. The fact that a completely positive map can be decomposed in the form (2.22) and the reverse statement 
was proved by Krauss [81], the decomposition is then called the Krauss decomposition. From a physical point of view, the condition of completely positive maps is necessary because we can never know if there is another system interacting with our subsystem. Therefore it is better to have a map preserving positivity for the whole system.

Our goal is to analyze from a phenomenological point of view the consequences of a time evolution dictated by the Lindblad equation where our subsystem consists of neutrinos propagating in a varying matter profile. Therefore, the Hamiltonian we will consider is strictly time dependent. But, once the Quantum theory has been extensively tested since its early days, we know that the predictions of the commutator piece in (2.35) are in very good agreement with what is observed in the physical world. Therefore, the changes caused by the parameters of the Kossakowski matrix must be small. We shall consider the $a_{i j}$ coefficients to be constant in time, this is justified because, if the parameters increased with time, at some point the Open System effects should be as important as the subsystem dynamics. While, on the other hand, if they decreased in time, eventually, they would become complete negligible in such a manner that the constrains we shall find must be related to their initial conditions.

In chapter [4] we will explain in further detail how we dealt with the neutrinos travelling through a variable matter profile, which shall be identified as the Earth's. In a few words, the model we have developed consists of slicing the Earth in layers of constant density matter, in such a manner that the Hamiltonian in each layer is constant. So the evolution in equation (2.38) can be applied layer by layer and with the aid of the composition rule (2.19) we can match the final states of each layer as the initial states of the following one.

In order to guarantee a complete positive evolution we have to choose the parameters in the Kossakowski matrix $a$ in order to satisfy the following conditions

1) $a_{i i} \geq 0$, for all $i$;

2) (Sylvester's criterion) The determinant of all upper-left sub-matrices must be positive;

3) Since the $a_{i j}$ are inherited from a scalar product, the Cauchy-Schwarz inequality, $a_{i i} a_{j j} \geq$ $\left|a_{i j}\right|^{2}$, must hold.

We will see that for $2 \times 2$ matrices we can find conditions that allow us to write direct the Lindbladian [82] assuring that it satisfies all the required properties. For bigger matrices the conditions become very intricate and, apart from special cases, it is better to work with all coefficients of the Kossakowski matrix instead. 


\subsubsection{Quantum Entropies}

The entropy of a state is a good quantifier to study its properties, here we will list some useful definitions.

1) The most common one is the von Neumann entropy, defined as:

Given a statistical ensemble described by the state $\rho$ the entropy (von Neumann) of the state is given by

$$
S(\rho)=-\operatorname{Tr}\{\rho \ln \rho\}
$$

few of its properties are:

(a) For any density operators the von Neumann entropy satisfies:

$$
S(\rho) \geq 0
$$

with the equality being valid only for pure states.

(b) For finite-dimensional systems, the von Neumann entropy is bounded from above

$$
S(\rho) \leq \ln N
$$

where $N$ is the dimension of the Hilbert space. Equality being valid only for maximally mixed states.

2) Relative entropy:

Given two states $\rho$ and $\sigma$, their relative entropy is given by

$$
S(\rho \| \sigma)=\operatorname{Tr}\{\rho \ln \rho\}-\operatorname{Tr}\{\rho \ln \sigma\}
$$

One of its properties is the fact that for all $\rho$ and $\sigma$ we have

$$
S(\rho \| \sigma) \geq 0
$$

where the equality holds only if $\sigma=\rho$. 
3) Linear entropy:

This entropy can be seen as a measure of the purity of the state, it is defined as

$$
S_{l}(\rho)=\operatorname{Tr}\left\{\rho-\rho^{2}\right\}=1-\operatorname{Tr}\left\{\rho^{2}\right\}
$$

From the properties of the density operator $\rho(2.1)$, it is easy to see that

$$
0 \leq S_{l}(\rho) \leq 1
$$

It is zero if and only if $\rho$ is pure. This quantifier can be used to study how the Lindbladian changes the purity of a system.

\subsection{Conclusions}

We aim to study neutrino oscillations as an Open Quantum System. To this purpose we have introduced in this chapter the formalism needed in order to achieve that.

We have shown that the evolution of the neutrino system, in this case, will be dictated by the Lindblad equation and the Open Quantum System effects will be parametrized by the elements of the Kossakowski matrix. These elements have to follow certain conditions to assure a complete positive evolution of the neutrino system.

Now we are in a position to use these theoretical results to calculate neutrino oscillation probabilities. In the next chapter, we will study neutrino oscillations in two and three generations with Open System effects. The two generations framework is an interesting starting point because we can find analytical expressions for the time evolution of the states even if the Open System effects are included. That makes this scenario ideal to show how to implement the theoretical tools we have developed. After discussing two generations we will move to the description in three generations considering also matter effects in order to study the consequences of the Open System effects in a real situation. 


\section{Part II}

\section{Phenomenology}





\section{CHAPTER 3}

\section{OPEN SYSTEM EFFECTS IN NEUTRINO OSCILLATIONS}

I $\mathrm{N}$ this chapter, we want to discuss the new effects which are present in the Open System framework. For this purpose we will start by discussing two neutrino oscillations in vacuum. We will do that in order to show how our framework works in a simple situation and how neutrino oscillations, even in two generations, are different for Dirac and Majorana neutrinos.

After that we will discuss neutrino oscillations in three generations in preparation for the next chapter where we will constrain the Open System effects with the atmospheric neutrino data from the IceCube collaboration.

The Lindbladian will always be implemented on the mass basis and the starting point for the Dissipator will always be the Kossakowski matrix (2.35), this makes the complete positivity condition easy to implement without the need to worry about impositions on the Lindbladian.

In the first section, we will show a general two-level system example, which is useful to understand the need to emphasize to define everything through the Kossakowksi matrix and then we will use this formalism to study neutrino oscillations in two and three generations in the second and third section of this chapter, respectively. 


\subsection{A general two-dimensional example}

In a two dimensional space we can expand the most general state $\rho$ using the identity matrix $\sigma_{0} \equiv \mathcal{I}_{2 \times 2}$ and the three Pauli matrices $\sigma_{i}, i=1,2,3$ as

$$
\rho=\left(\begin{array}{cc}
\rho_{0}+\rho_{3} & \rho_{1}-i \rho_{2} \\
\rho_{1}+i \rho_{2} & \rho_{0}-\rho_{3}
\end{array}\right) .
$$

The most general coefficient matrix $a_{i j}(2.35)$ is given by

$$
a=\left(\begin{array}{lll}
a_{11} & a_{12} & a_{13} \\
a_{21} & a_{22} & a_{23} \\
a_{31} & a_{32} & a_{33}
\end{array}\right)
$$

The operators $F_{i}, i=0,1,2,4$ can be chosen as ${ }^{1}$

$$
\begin{aligned}
& F_{0}=\frac{1}{\sqrt{2}} \mathcal{I}_{2 \times 2}, \\
& F_{i}=\frac{1}{\sqrt{2}} \sigma_{i} .
\end{aligned}
$$

Using this decomposition ${ }^{2}$ we can write the second term in (2.35) as

$$
\mathcal{D}[\rho(t)] \equiv \frac{1}{2} \sum_{i, j=1}^{3} a_{i j}\left(2 \sigma_{i} \rho \sigma_{j}-\left\{\sigma_{j} \sigma_{i}, \rho\right\}\right) .
$$

We will call $\mathcal{D}$ the Dissipator. Expanding all the operators in the basis $\left(\sigma_{0}, \sigma_{1}, \sigma_{2}, \sigma_{3}\right)$ and using repeatedly the anticommutation relations of the Pauli matrices we can show that the above equation can be written as (for the details see appendix B)

$$
\begin{aligned}
\mathcal{D}[\rho(t)]= & \left(2\left(\operatorname{Re}\left(a_{21}\right) \rho_{2}+\operatorname{Re}\left(a_{31}\right) \rho_{3}-a_{22} \rho_{1}-a_{33} \rho_{1}-2 \rho_{0} \operatorname{Im}\left(a_{32}\right)\right)\right) \sigma_{1} \\
& +\left(2\left(\operatorname{Re}\left(a_{12}\right) \rho_{1}+\operatorname{Re}\left(a_{32}\right) \rho_{3}-a_{11} \rho_{2}-a_{33} \rho_{2}-2 \rho_{0} \operatorname{Im}\left(a_{13}\right)\right)\right) \sigma_{2} \\
& +\left(2\left(\operatorname{Re}\left(a_{13}\right) \rho_{1}+\operatorname{Re}\left(a_{23}\right) \rho_{2}-a_{11} \rho_{3}-a_{22} \rho_{3}-2 \rho_{0} \operatorname{Im}\left(a_{21}\right)\right)\right) \sigma_{3} .
\end{aligned}
$$

Now we will perform the vectorization of the state $\rho$. This procedure consists of defining the vector $|\rho\rangle=\left(\rho_{0}, \rho_{1}, \rho_{2}, \rho_{3}\right)$ in the basis $\left(\sigma_{0}, \sigma_{1}, \sigma_{2}, \sigma_{3}\right)$ so that the action of $\mathcal{D}$ is defined

\footnotetext{
${ }^{1}$ Instead of defining $F_{N^{2}}$ as the operator proportional to the identity, we will use the index 0 so that $F_{0}=\frac{1}{\sqrt{N}} \mathcal{I}$

${ }^{2}$ The extra factor of $1 / 2$ will be absorbed in the $a_{i j}$ coefficients.
} 
through

$$
\mathcal{D}|\rho\rangle=-2\left(\begin{array}{cccc}
0 & 0 & 0 & 0 \\
2 \operatorname{Im}\left(a_{32}\right) & a_{22}+a_{33} & -\operatorname{Re}\left(a_{12}\right) & -\operatorname{Re}\left(a_{13}\right) \\
2 \operatorname{Im}\left(a_{13}\right) & -\operatorname{Re}\left(a_{12}\right) & a_{11}+a_{33} & -\operatorname{Re}\left(a_{23}\right) \\
2 \operatorname{Im}\left(a_{21}\right) & -\operatorname{Re}\left(a_{13}\right) & -\operatorname{Re}\left(a_{23}\right) & a_{11}+a_{22}
\end{array}\right)\left(\begin{array}{c}
\rho_{0} \\
\rho_{1} \\
\rho_{2} \\
\rho_{3}
\end{array}\right) .
$$

At this point, we have to impose that the von Neumann entropy in our subsystem must increase to avoid non-physical results, such as negative probabilities [83, 84]. A necessary and sufficient condition is that the first column of (3.6) must be equal to zero, which makes the matrix $a$ Hermitian (see appendix B).

Using the conditions 1), 2) and 3) from section 2.2.2, we can restrict directly the remaining coefficients in (3.6) and find the inequalities from [84, 82]. The operator $\mathcal{D}$ can then be written in the generic form [84], encountered in many papers in the literature

$$
\mathcal{D}=-2\left(\begin{array}{cccc}
0 & 0 & 0 & 0 \\
0 & a & b & c \\
0 & b & \alpha & \beta \\
0 & c & \beta & \gamma
\end{array}\right)
$$

This example is useful to see that the vectorized form has dimension $N^{2}$, so as we move to bigger spaces the complexity increases fast. Our ultimate goal is to study three generations of neutrinos in this framework, the corresponding time-evolving operator in this scenario is given by a $9 \times 9$ matrix, so instead of deriving intricate inequalities, we will work with the coefficients $a_{i j}$ directly, which automatically assures complete positivity if conditions 1), 2) and 3) are satisfied. Also using this simple example in two generations we have shown that we cannot use equation (3.7) directly without carefully taking into account the underlying formalism. A naive usage of the generic form can violate the important condition of complete positivity and also introduce a nonphysical Lindbladian which cannot be inherited from the mass basis [85].

Once we have (3.7) in vectorized form, it remains only to find the vectorized Hamiltonian to write the time evolution as

$$
\frac{\mathrm{d}|\rho\rangle}{\mathrm{d} t}=(\mathcal{H}+\mathcal{D})|\rho\rangle \equiv \mathcal{L}|\rho\rangle
$$

$\mathcal{H}$ is obtained by expanding all the operators in the commutator piece $-i[H, \rho(t)]$ in the $F_{i}$ basis and extracting the components of the vector $|\rho\rangle$. The evolved state is then given by (2.38). This is what makes the vectorization useful, the differential equation becomes linear. 


\subsection{Neutrino oscillations in two generations with Open Sys- tem effects}

Here we will analyze cases that have already been studied in the literature [86], but considering the definitions starting from equation (2.35). In two generations the neutrino Hamiltonian can be written in the mass basis as

$$
H=\left(\begin{array}{cc}
0 & 0 \\
0 & \frac{\Delta m^{2}}{2 E}
\end{array}\right),
$$

where $E$ is the neutrino energy and $\Delta m^{2}$ is the mass-squared difference between the neutrino mass eigenstates which will be fixed here, for illustration, to the atmospheric neutrino mass-squared value. The vectorized form can be easily found by performing the expansion of $-i[H, \rho(t)]$ in the basis $\left(\sigma_{0}, \sigma_{1}, \sigma_{2}, \sigma_{3}\right)$, to be

$$
\mathcal{H}=\left(\begin{array}{cccc}
0 & 0 & 0 & 0 \\
0 & 0 & \frac{\Delta m^{2}}{2 E} & 0 \\
0 & -\frac{\Delta m^{2}}{2 E} & 0 & 0 \\
0 & 0 & 0 & 0
\end{array}\right) .
$$

In [86] seven different cases were analyzed. Since here we just want to show how to use the formalism from a more fundamental starting point and to see the new features which arise when Open System effects are included, it suffices to revisit only cases (1), (4) and (5) (see table 3.1) from that reference. Since the Dissipator of case (1) is obtained by imposing that the average energy is conserved in the neutrino subsystem, before analyzing in detail the consequences of Open System effects for each scenario, we will study the validity of this restriction.

\subsubsection{Average energy conservation analysis}

The average energy in the density matrix formalism can be calculated by

$$
\langle H\rangle=\operatorname{Tr}\{\rho H\} .
$$

It is conserved if the following condition is satisfied

$$
\frac{\mathrm{d} \operatorname{Tr}\{H \rho\}}{\mathrm{d} t}=\operatorname{Tr}\{H \dot{\rho}\}=0
$$

Where the time derivative of the density operator is given by equation (2.35), with the replacement of $H$ by the two neutrino system Hamiltonian (3.9). In two generations the most 


\begin{tabular}{||cccc||}
\hline Cases & Dissipator & Kossakowski \\
\hline \hline (1) & $\mathcal{D}=\left(\begin{array}{cccc}0 & 0 & 0 & 0 \\
0 & -2 \gamma & 0 & 0 \\
0 & 0 & -2 \gamma & 0 \\
0 & 0 & 0 & 0\end{array}\right)$ & $a=\left(\begin{array}{ccc}0 & 0 & 0 \\
0 & 0 & 0 \\
0 & 0 & a_{33}\end{array}\right)$ \\
\hline (4) & $\mathcal{D}=\left(\begin{array}{cccc}0 & 0 & 0 & 0 \\
0 & -2 \gamma & 0 & 0 \\
0 & 0 & 0 & 0 \\
0 & 0 & 0 & -2 \gamma\end{array}\right)$ & $a=\left(\begin{array}{ccc}0 & 0 & 0 \\
0 & a_{22} & 0 \\
0 & 0 & 0\end{array}\right)$ \\
& $(5)$ & $\mathcal{D}=\left(\begin{array}{cccc}0 & 0 & 0 & 0 \\
0 & -2 \gamma & -\gamma & 0 \\
0 & -\gamma & -2 \gamma & 0 \\
0 & 0 & 0 & -2 \gamma\end{array}\right)$ & $a=\left(\begin{array}{ccc}a_{11} & a_{12} & 0 \\
a_{12} & a_{22} & 0 \\
0 & 0 & a_{33}\end{array}\right)$ \\
\hline
\end{tabular}

Table 3.1: Relation between the Dissipator and the Kossakowski matrix for each case studied in this section.

general mixing matrix can be written as

$$
U=\left(\begin{array}{cc}
\cos \theta & e^{i \alpha} \sin \theta \\
-e^{-i \alpha} \sin \theta & \cos \theta
\end{array}\right)
$$

where $\theta$ is the mixing angle and $\alpha$ the Majorana phase, which in general does not contribute for neutrino oscillations. However, as we shall see, when Open System effects are considered this fact does not hold anymore. The initial flavor states are written from the mass basis as (see equation (1.1))

$$
\rho_{\mu}(0)=U^{\dagger} \rho_{1}(0) U=\left(\begin{array}{cc}
\cos ^{2} \theta & e^{i \alpha} \sin \theta \cos \theta \\
e^{-i \alpha} \sin \theta \cos \theta & \sin ^{2} \theta
\end{array}\right),
$$

for the muon neutrino and

$$
\rho_{\tau}(0)=U^{\dagger} \rho_{2}(0) U=\left(\begin{array}{cc}
\sin ^{2} \theta & -e^{i \alpha} \sin \theta \cos \theta \\
-e^{-i \alpha} \sin \theta \cos \theta & \cos ^{2} \theta
\end{array}\right),
$$

for the tau neutrino. The states $\rho_{i}(0)=\left|\nu_{i}\right\rangle\left\langle\nu_{i}\right|,(i=1,2)$ are the initial states in mass basis.

The initial average energy value for each state can then be easily shown to be

$$
\begin{aligned}
& \langle H\rangle_{\mu}(0)=\operatorname{Tr}\left\{H \rho_{\mu}(0)\right\}=\frac{\sin ^{2} \theta \Delta m^{2}}{2 E}, \\
& \langle H\rangle_{\tau}(0)=\operatorname{Tr}\left\{H \rho_{\tau}(0)\right\}=\frac{\cos ^{2} \theta \Delta m^{2}}{2 E} .
\end{aligned}
$$


Considering the most general Kossakowski matrix in two generations from equation (3.2), the most general Dissipator can be written as

$$
\mathcal{D}=\left(\begin{array}{cccc}
0 & 0 & 0 & 0 \\
0 & -2\left(a_{22}+a_{33}\right) & 2 a_{12} & 2 a_{13} \\
0 & 2 a_{12} & -2\left(a_{11}+a_{33}\right) & 2 a_{23} \\
0 & 2 a_{13} & 2 a_{23} & -2\left(a_{11}+a_{22}\right)
\end{array}\right)
$$

which implies that

$$
\operatorname{Tr}\{\dot{\rho} H\}=-\frac{\rho_{1}(t) a_{13} \Delta m^{2}}{E}+\frac{\rho_{3}(t)\left(a_{11}+a_{22}\right) \Delta m^{2}}{E}-\frac{\rho_{2}(t) a_{23} \Delta m^{2}}{E},
$$

where $\rho_{1}(t), \rho_{2}(t), \rho_{3}(t)$ are the components of the density matrix operator in the vectorized form (3.6). Hence, assuming that the Open System parameters are energy independent, the imposition of energy conservation for all times leads to the restrictions

$$
\begin{aligned}
& a_{13}=a_{23}=0, \\
& a_{22}=-a_{11} .
\end{aligned}
$$

But equation (3.21) cannot be satisfied due to the condition 1), unless we have $a_{22}=0$. Also to guarantee complete positivity, by condition 2) we must also set $a_{12}=0$. Therefore, the only Dissipator that is consistent with energy conservation is

$$
\mathcal{D}=\left(\begin{array}{cccc}
0 & 0 & 0 & 0 \\
0 & -2 a_{33} & 0 & 0 \\
0 & 0 & -2 a_{33} & 0 \\
0 & 0 & 0 & 0
\end{array}\right) .
$$

This is exactly case (1) of [86] with $\gamma=a_{33}$.

\subsubsection{Discussion of Open System effects in two generations}

In the last subsection, we have found the Dissipator imposing restrictions on the Kossakowski matrix which led to the case (1), the only case where average energy is conserved. In general, if 1), 2), and 3 ) are satisfied, we could turn on any term in the Kossakowski matrix. We have developed a very useful tool to see how the Dissipator is modified when certain terms of the Kossakowski matrix are different from zero, the following Checker map: 


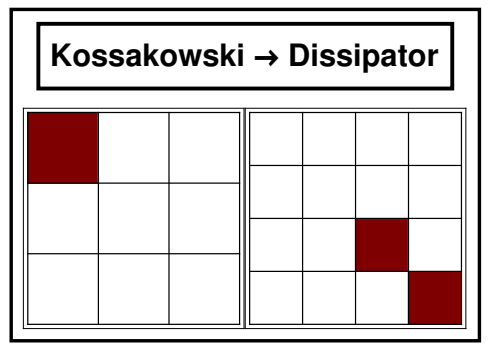

Figure 3.1: The Checker map, a useful tool to follow how an element of the Kossakowski matrix affects the Dissipator. On the left (the $3 \times 3$ matrix) is the Kossakowski matrix with only $a_{11}$ different from zero, on the right is the Dissipator corresponding to this Kossakowski matrix.

In appendix $\mathrm{C}$ a complete Checker map for all possibilities in two and three generations can be found. In two generations this map may seem redundant because we can see easily from equation (3.18) how the Dissipator will look like. However, in three generations this is not true anymore, the most general Dissipator can be very complex. Anyway, the present scenario serves as a simple way to see how the Checker map works.

Now we are going to discuss some features of the three cases from table 3.1. In what follows we will use the parameters values summarized in the tables 3.2 and 3.3 below.

\begin{tabular}{||cc||}
\hline Cases & Parameters \\
\hline \hline$(1)$ & $a_{33}=10^{-23} \mathrm{GeV}$ \\
\hline$(4)$ & $a_{22}=10^{-23} \mathrm{GeV}$ \\
\hline (5) & $a_{11}=2 a_{12}=a_{22}=a_{33}=10^{-23} \mathrm{GeV}$ \\
\hline
\end{tabular}

Table 3.2: Open system parameters used for each case.

In the table above the size of the matrix element is selected to be around the reach of the IceCube experiment. Since we can find analytic expressions for neutrino oscillations in two generations even with the Open System effects, we shall see that damping terms like $e^{-a_{i j} L}$ will often appear. Therefore, for the Open System parameters to have a sizeable effect we must have $a_{i j} L \sim 1$ which, for a baseline length equal to the Earth diameter, corresponds to $a_{i j} \sim$ $10^{-23} \mathrm{GeV}$.

\begin{tabular}{||cc||}
\hline Neutrino oscillation parameters & Values \\
\hline \hline $\sin ^{2} \theta$ & 0.55 \\
\hline $\mathrm{L}$ & $12742 \mathrm{~km}$ \\
\hline$\Delta m^{2}$ & $2.5 \times 10^{-3} \mathrm{eV}^{2}$ \\
\hline
\end{tabular}

Table 3.3: Neutrino parameters used for all the analysis in this section.

We have fixed the baseline length to the Earth diameter, the longest value possible for IceCube, the experiment we will analyze in the next chapter. Furthermore, we are using the mass- 
squared difference and the mixing angle corresponding to the neutrino oscillation parameters to which atmospheric neutrinos in IceCube are more sensitive to.

Let's start by discussing case (1). The evolved state can be found analytically in the present scenario $^{3}$

$$
\rho_{\mu}(L)=\left(\begin{array}{cc}
\cos ^{2} \theta & -e^{-2 a_{33} \mathrm{~L}+\mathrm{i} \alpha+\mathrm{i} \Delta \mathrm{L}} \cos \theta \sin \theta \\
-e^{-2 a_{33} \mathrm{~L}-\mathrm{i} \alpha-\mathrm{i} \Delta \mathrm{L}} \cos \theta \sin \theta & \sin ^{2} \theta
\end{array}\right)
$$

where we used the time baseline distance replacement, $t \rightarrow L$, valid for ultra-relativistic particles and adopted the shorthand notation $\Delta=\Delta m^{2} / 2 E$. Hence, since only the off-diagonal terms are affected, the net effect of this Dissipator is to destroy the coherence of the state. The survival probability can then be calculated using equation (1.11) with $t_{P}=0$ and $t_{D}=L$

$$
\mathcal{P}_{\nu_{\mu} \rightarrow \nu_{\mu}}(E)=\frac{1}{4}\left(3+\cos 4 \theta+2 e^{-2 a_{33} \mathrm{~L}} \cos \Delta \mathrm{L} \sin ^{2} 2 \theta\right)
$$

We can see that the standard probability is recovered in the limit $a_{33} \rightarrow 0$. At this point we can also note that the probability is independent of the phase $\alpha$, so in this scenario, just as in the standard one, the survival probability does not allow us to distinguish between Dirac and Majorana neutrinos.

We can see in the figure 3.2 how the Open System interactions change the oscillation pattern. For the region of interest for atmospheric neutrinos oscillations at IceCube we note that not only the oscillation peaks are damped but also the behavior in the high energy range is different for the standard and Open System scenarios. In particular, for energies above $E \sim 500 \mathrm{GeV}$ the standard probability is practically 1 while in case (1) the value is 0.64 .

The situation can also be studied with the aid of the entropy ${ }^{4}$. Since in the absence of Open System effects pure states always evolve into pure states the linear entropy for the standard scenario will always be zero, but as we can see from figure 3.3, the situation changes when Open System interactions are considered. In particular, in case (1), the entropy of the state always evolve to $S_{l}=0.46$.

Next let's discuss what happens in case (4). The survival probability is given by

\footnotetext{
${ }^{3}$ We will expose the results only for the muon neutrino since the result for the tau neutrino is obtained through the relation $\rho_{\tau}(t)=1-\rho_{\mu}(t)$.

${ }^{4}$ Since the behavior of the linear and Von Neumann entropies (see chapter [2]) is similar we will show only the linear entropy here.
} 


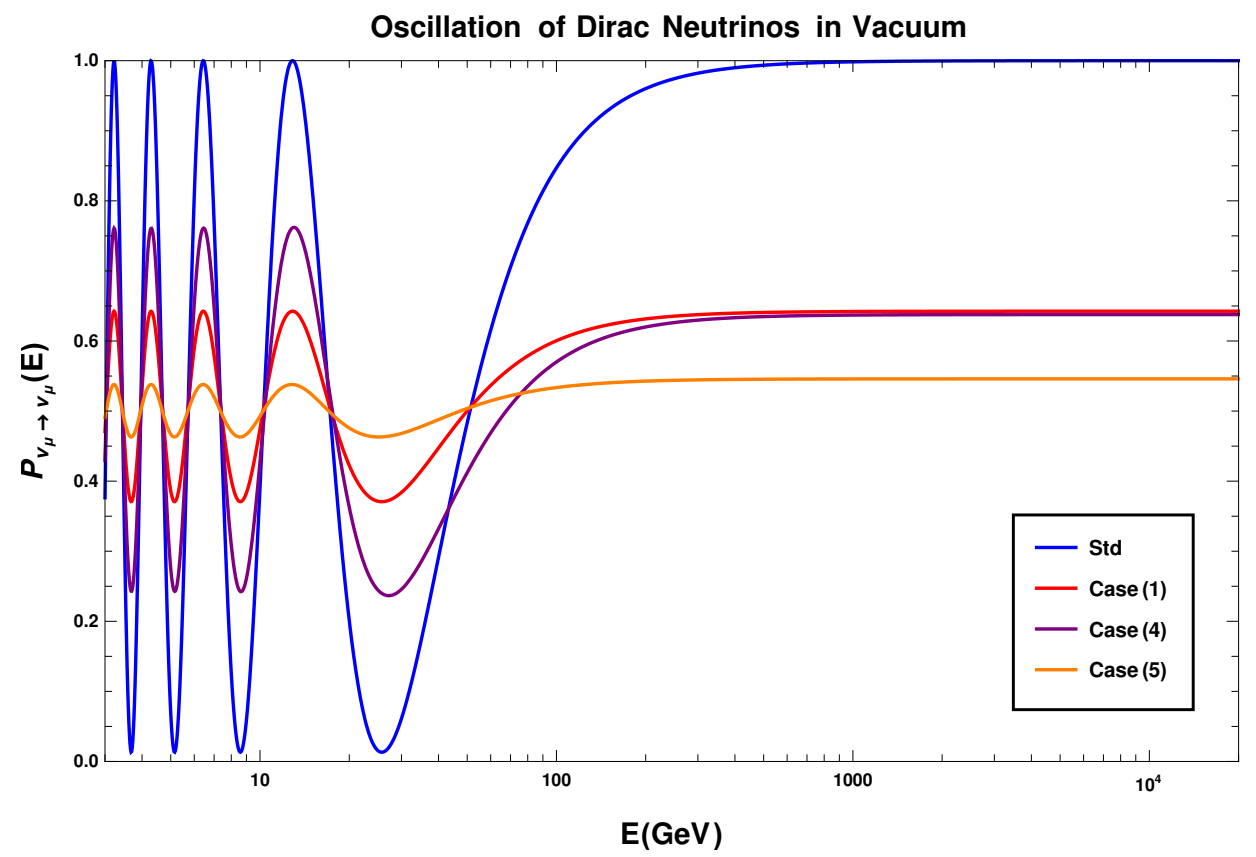

Figure 3.2: $\nu_{\mu}$ survival probability as a function of the energy for the parameters described in tables 3.2 and 3.3 for each case of table 3.1.

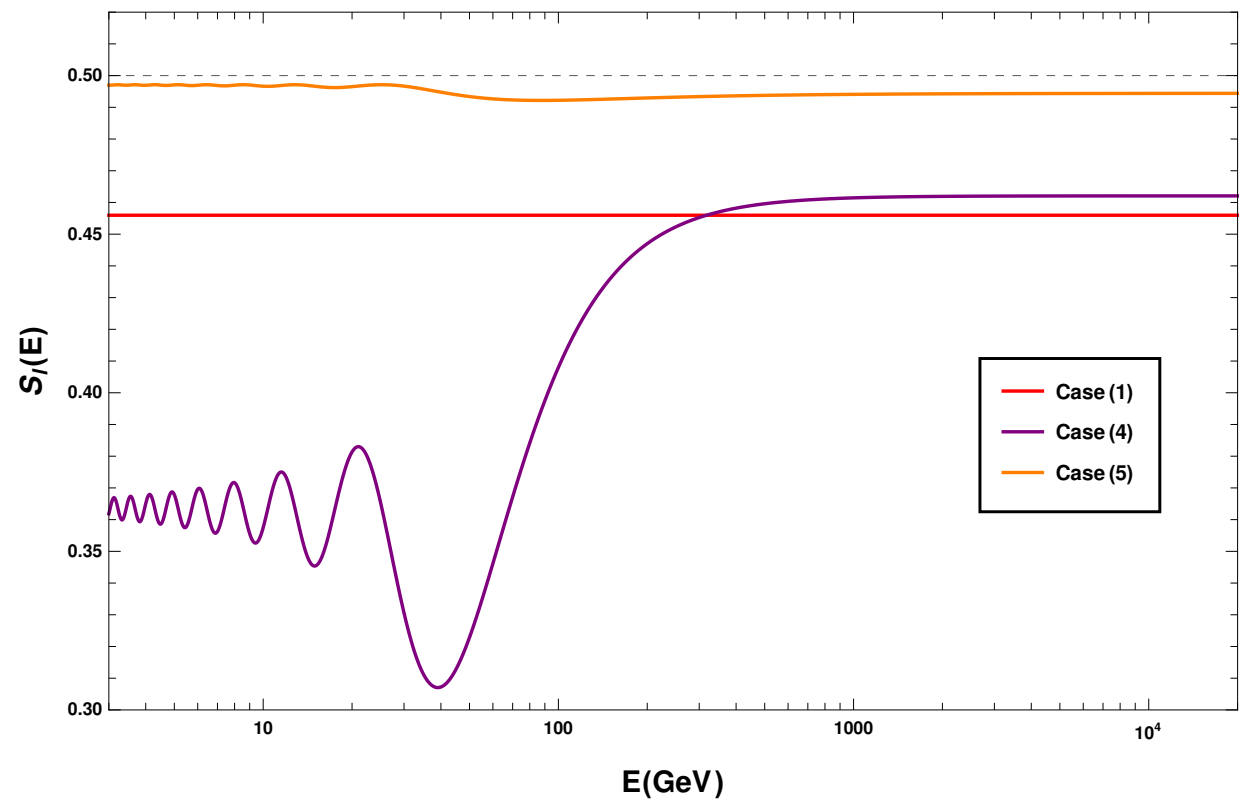

Figure 3.3: Linear entropy for the $\nu_{\mu}$ state considering the parameters from tables 3.2 and 3.3. Since in the standard scenario the state remains pure for all the energies the linear entropy is zero, therefore this case is not shown in the plot. 


$$
\begin{aligned}
\mathcal{P}_{\nu_{\mu} \rightarrow \nu_{\mu}}(E) & =\frac{1}{2}\left(1+e^{-2 a_{22} \mathrm{~L}} \cos ^{2} 2 \theta+e^{-a_{22} \mathrm{~L}}\left(4 \cos ^{2} \theta \cosh \sqrt{a_{22}^{2}-\Delta^{2}} \mathrm{~L}_{\sin ^{2} \theta}\right.\right. \\
& \left.\left.-\frac{a_{22} \cos 2 \alpha \sin ^{2} 2 \theta \sinh \sqrt{a_{22}^{2}-\Delta^{2}} \mathrm{~L}}{\sqrt{a_{22}^{2}-\Delta^{2}}}\right)\right)
\end{aligned}
$$

In this case we can see that the probability depends on the Majorana phase $\alpha$, so at least at the probability level, we can distinguish the nature of the neutrino. On the other hand, the dependence is through an even function which assures that the CP and CPT symmetries are still conserved. From figure 3.2 we can infer that the oscillation peaks for this case are less affected than in the previous case, but in the high energy range the probability is practically indistinguishable from case (1).

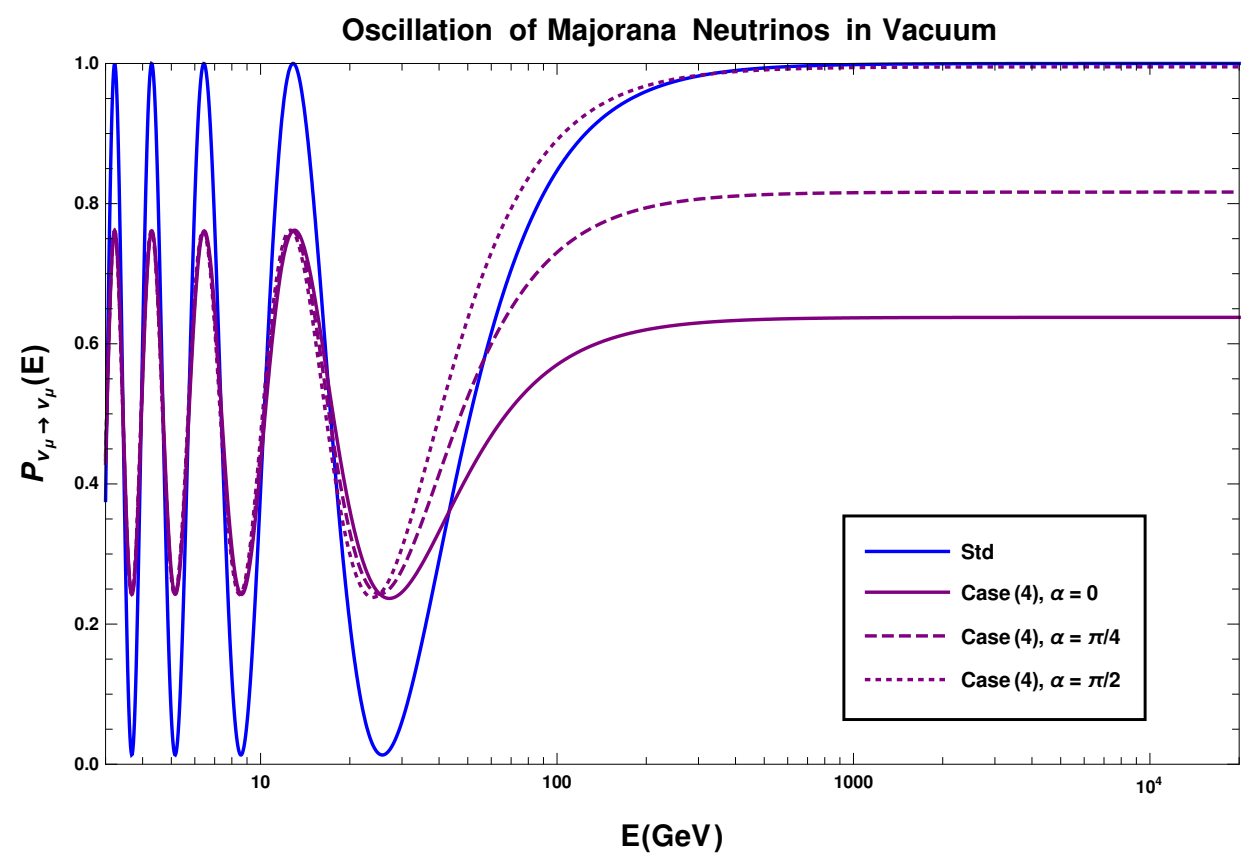

Figure 3.4: Same as figure 3.2, but considering different values of the Majorana phase $\alpha$.

On the other hand, from figure 3.4 we can see that for different values of the Majorana phase $\alpha$ we can reduce even more the effect of the Open System interactions, in particular for $\alpha=\pi / 2$ the behavior for high energies approaches the standard scenario.

A new feature of this case is the fact that the Open System interactions affect also the populations of the density matrix, for instance, the first diagonal element of the density operator is 


$$
\rho_{\mu}^{11}(L)=\frac{1}{2}\left(1+e^{-2 a_{22} \mathrm{~L}} \cos 2 \theta\right) .
$$

Therefore, for $L$ equal to the diameter of the Earth and $a_{22} \geq 1.6 \times 10^{-23} \mathrm{GeV}$, the Open System effects would drive the state to a maximally mixed one.

The linear entropy analysis in this scenario is also helpful to better understand what is happening in the neutrino subsystem. We can see in figure 3.5 that since for $\alpha=\pi / 2$ the Open System effects are diminished the state evolves into a more pure one while for the case where the effects are bigger for $\alpha=0$, the Open System interactions drive the state towards a nearly maximally mixed one.

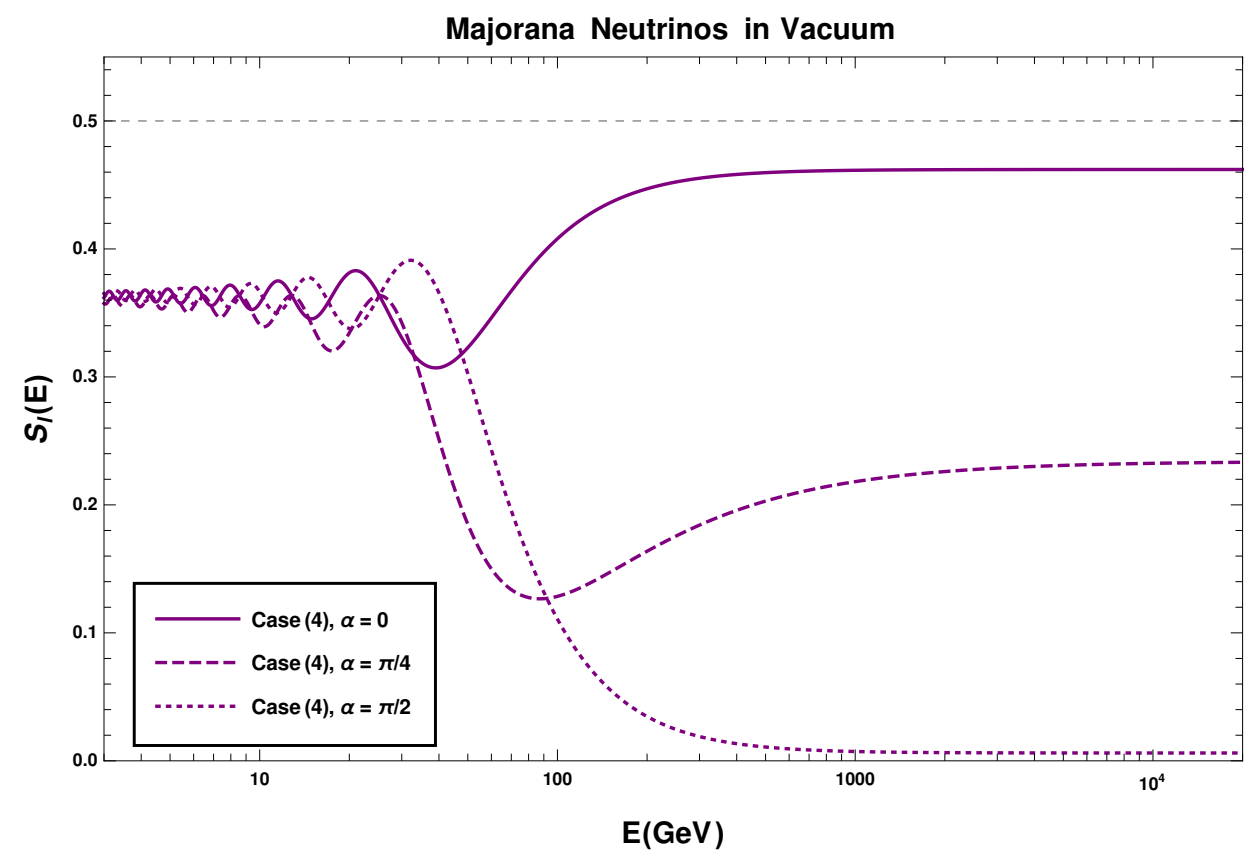

Figure 3.5: Same as figure 3.3, but considering different values of the Majorana phase $\alpha$.

Finally, we will comment on case (5). The probability is given by

$$
\begin{aligned}
\mathcal{P}_{\nu_{\mu} \rightarrow \nu_{\mu}}(E) & =\frac{1}{2}+\frac{1}{2} e^{-2\left(a_{11}+a_{22}\right) \mathrm{L}} \cos ^{2} 2 \theta+\frac{1}{\omega} e^{-\left(a_{11}+a_{22}+2 a_{33}+\omega\right) \mathrm{L}} \cos ^{2} \theta \sin ^{2} \theta \\
& \times\left(\left(1+e^{2 \omega \mathrm{L}}\right) \omega+\left(a_{22}-a_{11}\right) \cos 2 \alpha+2 a_{12} \sin 2 \alpha\right. \\
& \left.+e^{2 \omega \mathrm{L}}\left(\left(a_{11}-a_{22}\right) \cos 2 \alpha-2 a_{12} \sin 2 \alpha\right)\right)
\end{aligned}
$$

where we defined $\omega=\sqrt{4 a_{12}^{2}+\left(a_{11}-a_{22}\right)^{2}-\Delta^{2}}$. In the above equation we can readily see that, like case (4), it is sensitive to the Majorana phase, the difference is that in here there is an odd $\alpha$-dependent function, which implies that the CP symmetry condition 


$$
\Delta \mathcal{P}_{\nu_{\alpha} \rightarrow \nu_{\beta}}(E) \equiv \mathcal{P}_{\nu_{\alpha} \rightarrow \nu_{\beta}}(E)-\mathcal{P}_{\bar{\nu}_{\alpha} \rightarrow \bar{\nu}_{\beta}}(E)=0
$$

is violated. Furthermore, in (3.28), considering the $\nu_{\mu} \rightarrow \nu_{\mu}$ channel the symmetry that is violated is actually the CPT symmetry. This is an important consequence since IceCube can measure both neutrinos and antineutrinos. Note that in the standard scenario, even in three generations in vacuum, CP and CPT are conserved in neutrino survival probabilities. However, when Open System effects are at play this is not the case.

The entropy analysis in this scenario is trivial, since the Open System effects drive the state into a maximally mixed one in such a way that $S_{l} \approx 0.5$, independent of the Majorana phase $\alpha$.

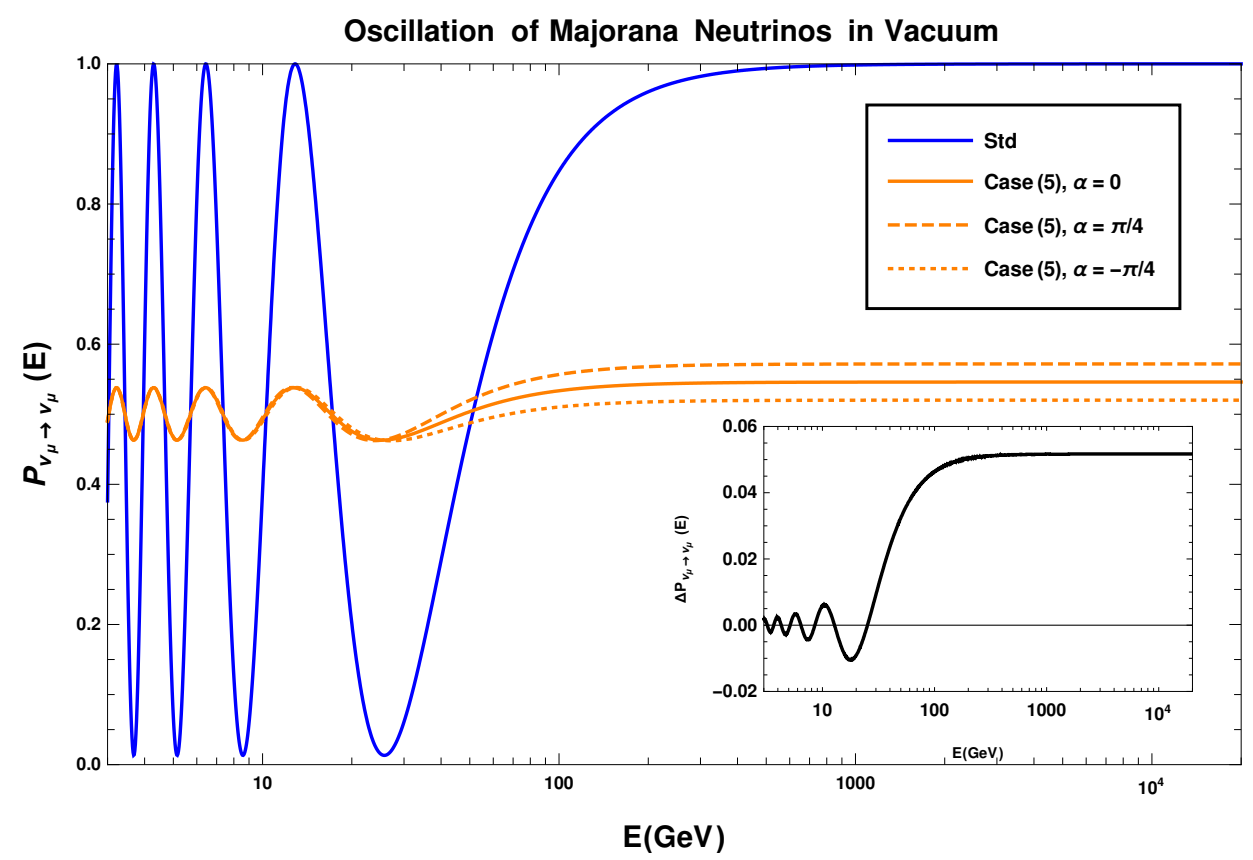

Figure 3.6: Same as figure 3.2, but considering different values of the Majorana phase $\alpha$. The smaller plot shows the amount of CP (CPT) violation due to Open System effects (see equation (3.28)).

\subsection{Neutrino oscillations in three generations with Open Sys- tem effects}

Now that we have understood the effect the Open System parameters can have in the neutrino subsystem in vacuum using the two generations description, we are ready to tackle the more realistic situation involving three generations in matter. 
For this purpose let's recall a few results we have discussed in chapter [1]. In three generations, we can write the PMNS matrix as

$$
U=U_{23} U_{13} U_{12} U_{\mathrm{Maj}}
$$

where

$$
U_{23}=\left(\begin{array}{ccc}
1 & 0 & 0 \\
0 & c_{23} & s_{23} \\
0 & -s_{23} & c_{23}
\end{array}\right), \quad U_{13}=\left(\begin{array}{ccc}
c_{13} & 0 & s_{13} e^{-i \delta} \\
0 & 1 & 0 \\
-s_{13} e^{i \delta} & 0 & c_{13}
\end{array}\right), \quad U_{12}=\left(\begin{array}{ccc}
c_{12} & s_{12} & 0 \\
-s_{12} & c_{12} & 0 \\
0 & 0 & 1
\end{array}\right)
$$

and

$$
U_{\text {Maj }}=\left(\begin{array}{ccc}
1 & 0 & 0 \\
0 & e^{i \phi_{1}} & 0 \\
0 & 0 & e^{i \phi_{2}}
\end{array}\right)
$$

encodes the two possible Majorana phases in the three generation description. We adopted the notation $c_{i j}\left(s_{i j}\right)=\cos \theta_{i j}\left(\sin \theta_{i j}\right)$. The Hamiltonian in the mass basis, considering matter effects, is then given by

$$
H_{M}=H+U^{\dagger} V U
$$

where

$$
V= \pm \sqrt{2} G_{F}\left(\begin{array}{ccc}
n_{e} & 0 & 0 \\
0 & 0 & 0 \\
0 & 0 & 0
\end{array}\right)
$$

and

$$
H=\left(\begin{array}{ccc}
0 & 0 & 0 \\
0 & \frac{\Delta m_{21}^{2}}{2 E} & 0 \\
0 & 0 & \frac{\Delta m_{31}^{2}}{2 E}
\end{array}\right)
$$

Recall that $n_{e}$ is the electron number density and that the + sign in (3.32) is for neutrinos while the - sign is for antineutrinos. To include the Open System effects we must also find the most general Dissipator. In three dimensions the Kossakowksi matrix can be written as 


$$
a=\left(\begin{array}{llllllll}
a_{11} & a_{12} & a_{13} & a_{14} & a_{15} & a_{16} & a_{17} & a_{18} \\
a_{21} & a_{22} & a_{23} & a_{24} & a_{25} & a_{26} & a_{27} & a_{28} \\
a_{31} & a_{32} & a_{33} & a_{34} & a_{35} & a_{36} & a_{37} & a_{38} \\
a_{41} & a_{42} & a_{43} & a_{44} & a_{45} & a_{46} & a_{47} & a_{48} \\
a_{51} & a_{52} & a_{53} & a_{54} & a_{55} & a_{56} & a_{57} & a_{58} \\
a_{61} & a_{62} & a_{63} & a_{64} & a_{65} & a_{66} & a_{67} & a_{68} \\
a_{71} & a_{72} & a_{73} & a_{74} & a_{75} & a_{76} & a_{77} & a_{78} \\
a_{81} & a_{82} & a_{83} & a_{84} & a_{85} & a_{86} & a_{87} & a_{88}
\end{array}\right),
$$

after imposing the entropy to increase we also have the following constraint $a_{i j}=a_{j i}$. Under this assumption, analogously to equation (3.6), the most general Dissipator can then be written as

$$
\mathcal{D}=\left(\begin{array}{ccccccccc}
0 & 0 & 0 & 0 & 0 & 0 & 0 & 0 & 0 \\
0 & \mathcal{D}_{11} & \mathcal{D}_{12} & \mathcal{D}_{13} & \mathcal{D}_{14} & \mathcal{D}_{15} & \mathcal{D}_{16} & \mathcal{D}_{17} & \mathcal{D}_{18} \\
0 & \mathcal{D}_{12} & \mathcal{D}_{22} & \mathcal{D}_{23} & \mathcal{D}_{24} & \mathcal{D}_{25} & \mathcal{D}_{26} & \mathcal{D}_{27} & \mathcal{D}_{28} \\
0 & \mathcal{D}_{13} & \mathcal{D}_{23} & \mathcal{D}_{33} & \mathcal{D}_{34} & \mathcal{D}_{35} & \mathcal{D}_{36} & \mathcal{D}_{37} & \mathcal{D}_{38} \\
0 & \mathcal{D}_{14} & \mathcal{D}_{24} & \mathcal{D}_{34} & \mathcal{D}_{44} & \mathcal{D}_{45} & \mathcal{D}_{46} & \mathcal{D}_{47} & \mathcal{D}_{48} \\
0 & \mathcal{D}_{15} & \mathcal{D}_{25} & \mathcal{D}_{35} & \mathcal{D}_{45} & \mathcal{D}_{55} & \mathcal{D}_{56} & \mathcal{D}_{57} & \mathcal{D}_{58} \\
0 & \mathcal{D}_{16} & \mathcal{D}_{26} & \mathcal{D}_{36} & \mathcal{D}_{46} & \mathcal{D}_{56} & \mathcal{D}_{66} & \mathcal{D}_{67} & \mathcal{D}_{68} \\
0 & \mathcal{D}_{17} & \mathcal{D}_{27} & \mathcal{D}_{37} & \mathcal{D}_{47} & \mathcal{D}_{57} & \mathcal{D}_{67} & \mathcal{D}_{77} & \mathcal{D}_{78} \\
0 & \mathcal{D}_{18} & \mathcal{D}_{28} & \mathcal{D}_{38} & \mathcal{D}_{48} & \mathcal{D}_{58} & \mathcal{D}_{68} & \mathcal{D}_{78} & \mathcal{D}_{88}
\end{array}\right)
$$

where the matrix elements are shown in appendix $\mathrm{C}$.

In this work we will consider a five-layer description of the Earth ${ }^{5}$ [87], the model will be discussed in more detail in the next chapter. The parameters characterizing each layer are given in table 3.4 and were obtained from the Preliminary Earth Reference Model (PREM) [88, 87].

The initial flavor states can then be obtained from the mass basis

$$
\begin{aligned}
& \rho_{e}(0)=U^{\dagger} \rho_{1}(0) U, \\
& \rho_{\mu}(0)=U^{\dagger} \rho_{1}(0) U, \\
& \rho_{\tau}(0)=U^{\dagger} \rho_{1}(0) U,
\end{aligned}
$$

where

\footnotetext{
${ }^{5}$ This implies that a neutrino can cross $1,3,5,7$, or 9 layers, depending on the zenith angle.
} 


\begin{tabular}{|c|c|c|}
\hline Layer $i$ & External radius (in units of Earth radius) & $n_{e}$ of $i$ th layer $\left(\mathrm{GeV}^{3}\right)$ \\
\hline 1 & 1 & $7.78 \times 10^{-18}$ \\
\hline 2 & 0.937 & $8.84 \times 10^{-18}$ \\
\hline 3 & 0.895 & $1.14 \times 10^{-17}$ \\
\hline 4 & 0.546 & $2.41 \times 10^{-17}$ \\
\hline 5 & 0.192 & $2.79 \times 10^{-17}$ \\
\hline
\end{tabular}

Table 3.4: Parameters characterizing each Earth layer. The layer radius is written as a fraction of the Earth radius $R_{E}=6371 \mathrm{~km}$ and values for the electron number density $n_{e}$ are based on the Preliminary Earth Reference Model (PREM) [88]. The units are chosen to give the exact numbers we shall use in the time evolution of the neutrino subsystem.

$$
\rho_{1}(0)=\left(\begin{array}{lll}
1 & 0 & 0 \\
0 & 0 & 0 \\
0 & 0 & 0
\end{array}\right), \quad \rho_{2}(0)=\left(\begin{array}{lll}
0 & 0 & 0 \\
0 & 1 & 0 \\
0 & 0 & 0
\end{array}\right), \quad \rho_{3}(0)=\left(\begin{array}{lll}
0 & 0 & 0 \\
0 & 0 & 0 \\
0 & 0 & 1
\end{array}\right),
$$

are the initial mass eigenstates. Let the initial state be located at $L=L_{i}$, then the most general oscillation probability is a function of both the energy $E$ and the baseline distance $L$, it is given by

$$
\mathcal{P}_{\alpha \rightarrow \beta}(L, E)=\operatorname{Tr}\left\{\rho_{\alpha}\left(L_{i}\right) \rho_{\beta}(L)\right\}
$$

In this section, we will also keep the baseline distance $L$ fixed to the biggest baseline distance of the IceCube experiment. This is useful to show why IceCube is a clever choice to search for Open System effects. How to handle the real situation in which the neutrinos travel through the Earth in different paths before reaching the IceCube detector will be shown in the next chapter. Besides the Earth related parameters summarized in table 3.4 we will also use the oscillation parameters, for normal ordering, summarized in table 3.5. Except for the $\delta$ phase, the remaining parameters are fixed to the latest NuFIT release values ${ }^{6}$ [89]. We chose to use $\delta=3 \pi / 2$ in this section, because it is close to the best NuFIT value and other experiments such as $\mathrm{T} 2 \mathrm{~K}[90]$ seem to be pointing to this value.

In the present situation the $F_{i}$ can be chosen to be the Gell Mann matrices plus the three dimensional identity matrix. With this modification, the vectorization of $H_{M}$ in equation (1.47) can be performed by following the exact same steps of section 3.1. The evolved flavor state $\alpha$ is then given by

\footnotetext{
${ }^{6}$ Available online on http://www.nu-fit.org/.
} 


\begin{tabular}{|c|c|}
\hline Neutrino oscillation parameters & Value \\
\hline $\sin ^{2} \theta_{12}$ & 0.310 \\
\hline $\sin ^{2} \theta_{23}$ & 0.558 \\
\hline $\sin ^{2} \theta_{13}$ & 0.02241 \\
\hline$\delta$ & $3 \pi / 2$ \\
\hline$\Delta m_{21}^{2}$ & $7.39 \times 10^{-5} \mathrm{eV}^{2}$ \\
\hline$\Delta m_{31}^{2}$ & $2.523 \times 10^{-3} \mathrm{eV}^{2}$ \\
\hline$L$ & $12742 \mathrm{~km}$ \\
\hline
\end{tabular}

Table 3.5: Neutrino oscillation parameters, except for the $\delta$ phase, correspond to the best fit values from the NuFIT release [89]. The baseline distance $L$ is fixed to the biggest value possible for the IceCube experiment.

$$
\rho_{\alpha}(L)=e^{\mathcal{L} L} \rho_{\alpha}\left(L_{i}\right)
$$

where $\mathcal{L}=\mathcal{H}+\mathcal{D}$. Now that we have the general picture, we will study the two particular Kossakowski matrices below,

$$
a=\left(\begin{array}{cccccccc}
0 & 0 & 0 & 0 & 0 & 0 & 0 & 0 \\
0 & 0 & 0 & 0 & 0 & 0 & 0 & 0 \\
0 & 0 & 0 & 0 & 0 & 0 & 0 & 0 \\
0 & 0 & 0 & a_{44} & 0 & 0 & 0 & 0 \\
0 & 0 & 0 & 0 & 0 & 0 & 0 & 0 \\
0 & 0 & 0 & 0 & 0 & 0 & 0 & 0 \\
0 & 0 & 0 & 0 & 0 & 0 & 0 & 0 \\
0 & 0 & 0 & 0 & 0 & 0 & 0 & 0
\end{array}\right), \quad \tilde{a}=\left(\begin{array}{cccccccc}
a_{11} & a_{12} & 0 & 0 & 0 & 0 & 0 & 0 \\
a_{12} & a_{22} & 0 & 0 & 0 & 0 & 0 & 0 \\
0 & 0 & 0 & 0 & 0 & 0 & 0 & 0 \\
0 & 0 & 0 & 0 & 0 & 0 & 0 & 0 \\
0 & 0 & 0 & 0 & 0 & 0 & 0 & 0 \\
0 & 0 & 0 & 0 & 0 & 0 & 0 & 0 \\
0 & 0 & 0 & 0 & 0 & 0 & 0 & 0 \\
0 & 0 & 0 & 0 & 0 & 0 & 0 & 0
\end{array}\right) .
$$

This choice was guided by the Checker map (see appendix C) and the intuition formed in the last section. We want to illustrate the consequences of the Open System effects by showing simple cases. Since off Diagonal terms on the Dissipator resulted in CP and CPT violation, we chose the two cases in such a manner that the corresponding Dissipator is not only a diagonal matrix. Each of these two cases are sensitive only to one of the Majorana phases, so in order to show that we can have effect of both $\phi_{1}$ and $\phi_{2}$ we must consider both scenarios. Because the first case requires less parameters in the Kossakowski matrix, we used the other matrix $\tilde{a}$ from equation (3.39) only in the situations where $\phi_{1}$ is assumed to be different from zero. The values of the Open System parameters are shown in the table below 


\begin{tabular}{|c|c|}
\hline Open System parameters & Value \\
\hline$a_{11}=a_{22}=2 a_{12}=a_{44}$ & $10^{-23} \mathrm{GeV}$ \\
\hline
\end{tabular}

Table 3.6: Open System parameters used for the analysis of the two Dissipators considered in this section.

Motivated by our ultimately goal, the study of atmospheric neutrinos in the IceCube experiment, we will focus on all the possible $\nu_{\mu}$ oscillation channels: $\nu_{\mu} \rightarrow \nu_{\mu}, \nu_{\mu} \rightarrow \nu_{e}$ and $\nu_{\mu} \rightarrow \nu_{\tau}$.

First, let's start by investigating the effect of varying the $\delta$ phase in the oscillation probabilities. For this purpose, the two Majorana phases are set to zero. It is clear from figures 3.7, 3.8 and 3.9, that in the presence of Open System effects, the value of the $\delta$ phase can change the oscillation pattern.

The consequences for the $\nu_{\mu} \rightarrow \nu_{\mu}$ survival probability is very similar to what happened in the two generation description. The first oscillation peak is damped and we have a depletion in the asymptotic value in comparison to the standard scenario where the survival probability is basically one for energies above $E \geq 500 \mathrm{GeV}$. A very similar damping effect is observed in the first peak of the $\nu_{\mu} \rightarrow \nu_{\tau}$ channel (see figure 3.9), but for high energies the $\nu_{\tau}$ production is slightly enhanced instead. One can wonder what happened to the missing $\nu_{\mu}$ and $\nu_{\tau}$ neutrinos. From figure 3.8 we can conclude that the $\nu_{\mu} \rightarrow \nu_{e}$ channel is dramatically enhanced by the Open System effects. Since in the IceCube experiment the $\nu_{e}$ and $\nu_{\tau}$ neutrino events have different signatures in the detector from the $\nu_{\mu}$ neutrino events (see section 4.1), one possibility for the experiment to test the Open System predictions is by counting number of events that happened for each possible topology and compare with the standard prediction.

In addition to the $\delta$ phase we can see in figures 3.10, 3.11 and 3.12 that both $\phi_{1}$ and $\phi_{2}$ also affect the oscillation pattern in the Open System framework. In particular, for $\phi_{2}=\pi / 4$ we would have even more $\nu_{e}$ reaching the IceCube detector.

The dependence on the phases implies that CP and CPT are violated. As one can see in figures 3.13, 3.14 and 3.15, due to matter effects, even in the standard scenario with $\delta=0$ we would have CP and CPT violation. It is hard to disentangle the contribution of matter and Open System effects to the CPT (CP) violation for small energies but since as the energy increases the less the matter effects are important, we can look at energies above $E \geq 50 \mathrm{GeV}$ where the violation is caused solely by the Open System interactions.

Similarly to what we did in the two generation description we can also use the linear entropy to study the Open System effects. Since the modifications are very similar to what we found in the last section we will exhibit only two different plots 3.16 and 3.17. As expected from the 
study of the linear entropy in the two generation framework, the Open System effects drive the initial pure state into a mixed one. Furthermore, we can see that the Majorana phases can alter how mixed the final state is. For instance, a Majorana muon neutrino with $\phi_{2}=\pi / 4$ would end up in a more mixed state in comparison to a Dirac muon neutrino for energies $E>60 \mathrm{GeV}$.

Finally, in order to compare the reach of the IceCube experiment with other experiments in figure 3.18 we exhibit the sensitivity of the $\mathrm{NO} \nu \mathrm{A}$ experiment, the current active laboratory experiment with the longest baseline length, to Open System effects. We can see that the Open System parameter have a sizeable effect at the probability level for $a_{i j} \sim 10^{-22} \mathrm{GeV}$ considering the reach of the $\mathrm{NO} \nu \mathrm{A}$ experiment, which makes the IceCube experiment more sensitive to this kind of effects.

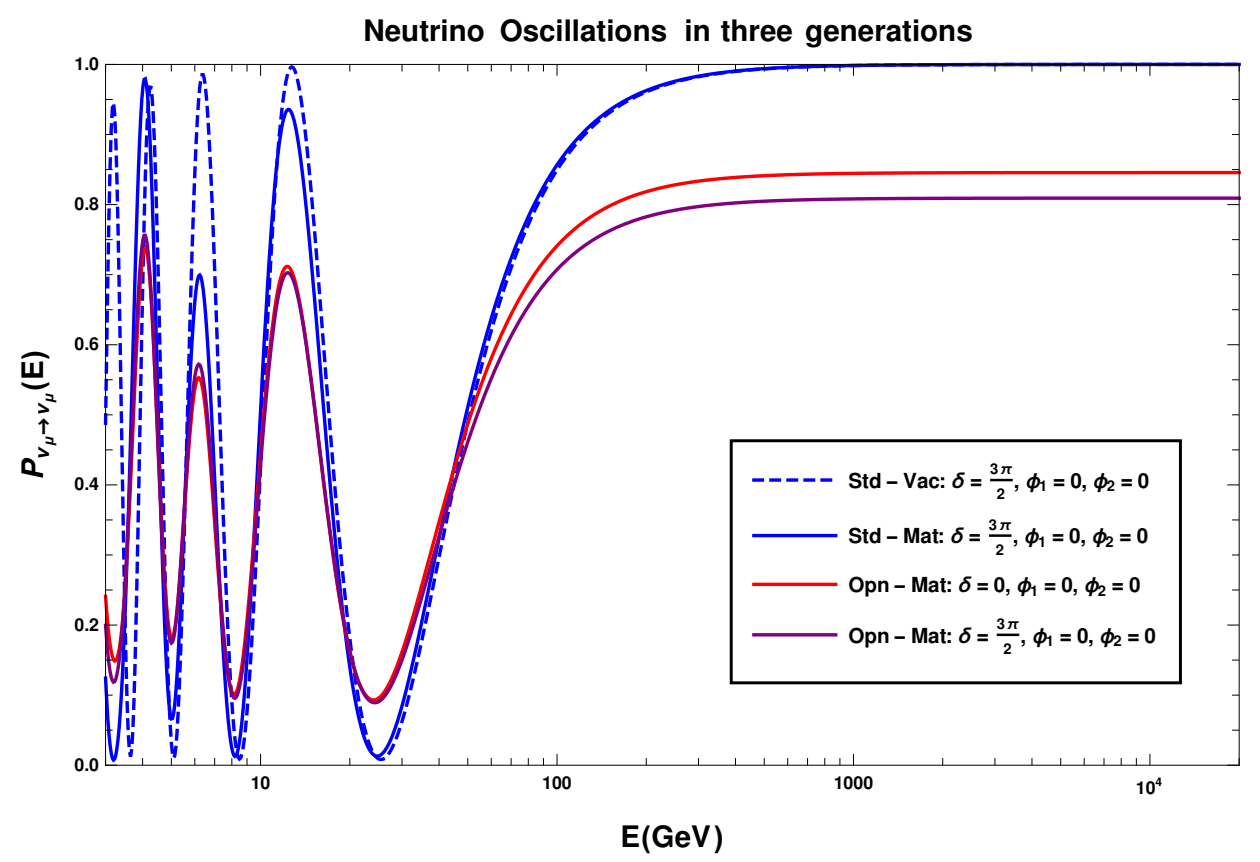

Figure 3.7: The $\nu_{\mu} \rightarrow \nu_{\mu}$ oscillation channel considering different values for the $\delta$ phase and setting the two Majorana phases to zero. The parameters used are summarized in tables 3.4, 3.5 and 3.6. The Opn, Std and Mat abbreviations stand for open, standard and matter, respectively. All the Open System cases were obtained by considering the Kossakowski matrix $a$ in equation (3.39). 


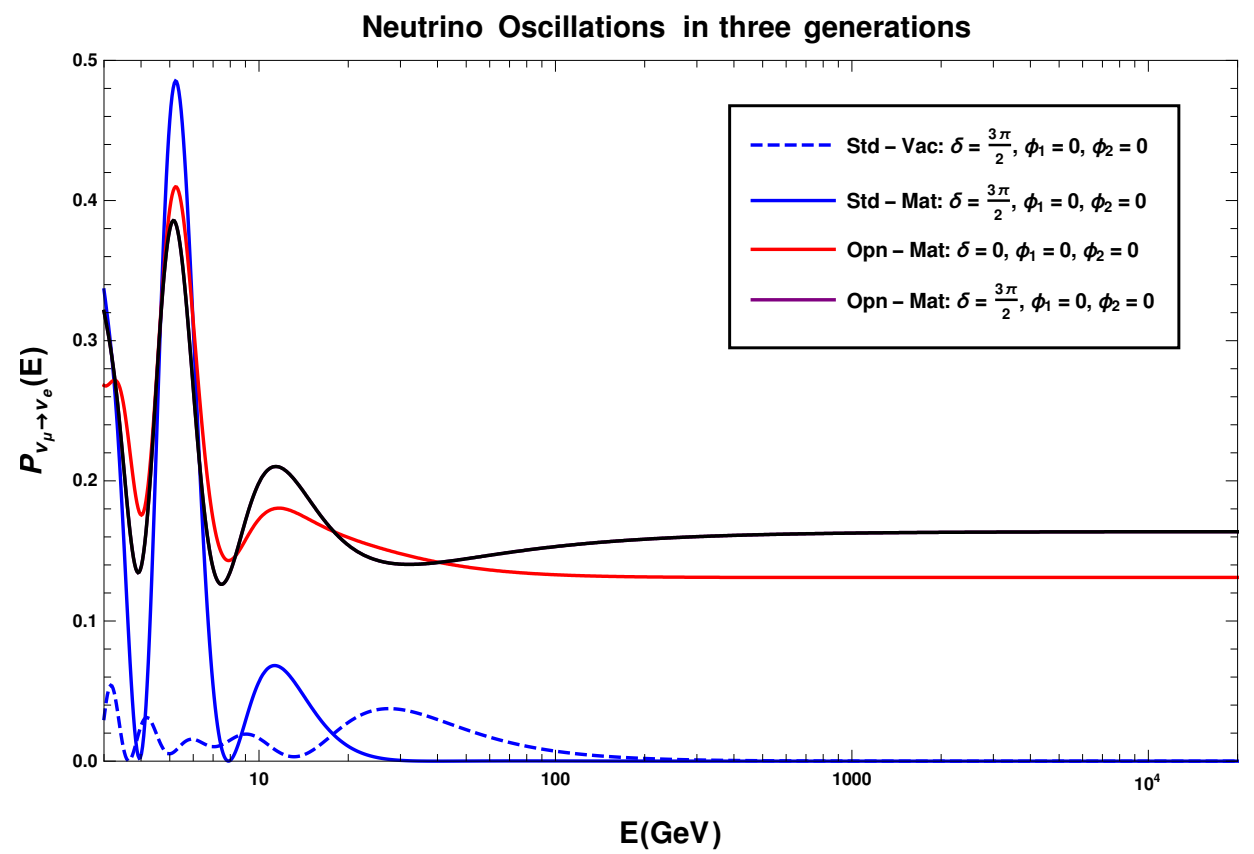

Figure 3.8: Same as figure 3.7, but considering the $\nu_{\mu} \rightarrow \nu_{e}$ oscillation channel.

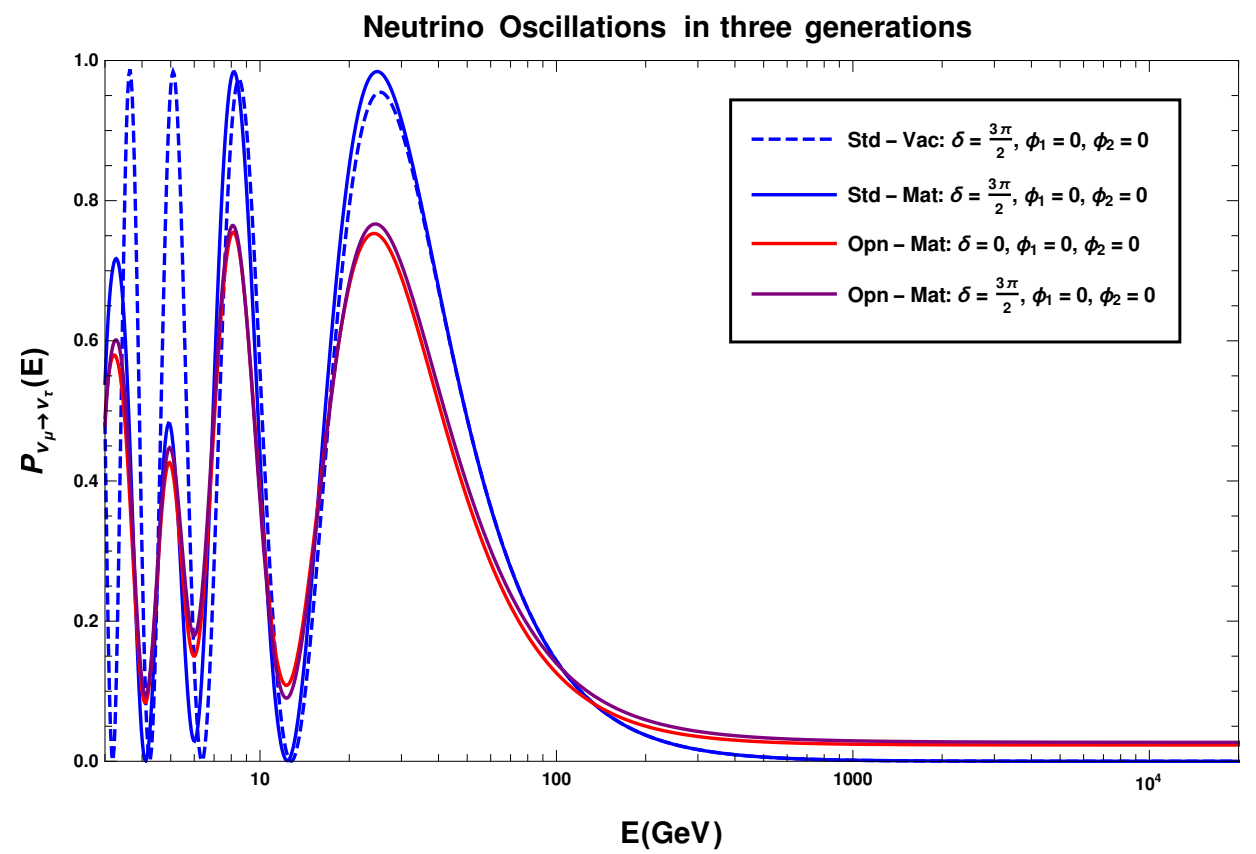

Figure 3.9: Same as figure 3.7, but considering the $\nu_{\mu} \rightarrow \nu_{\tau}$ oscillation channel. 


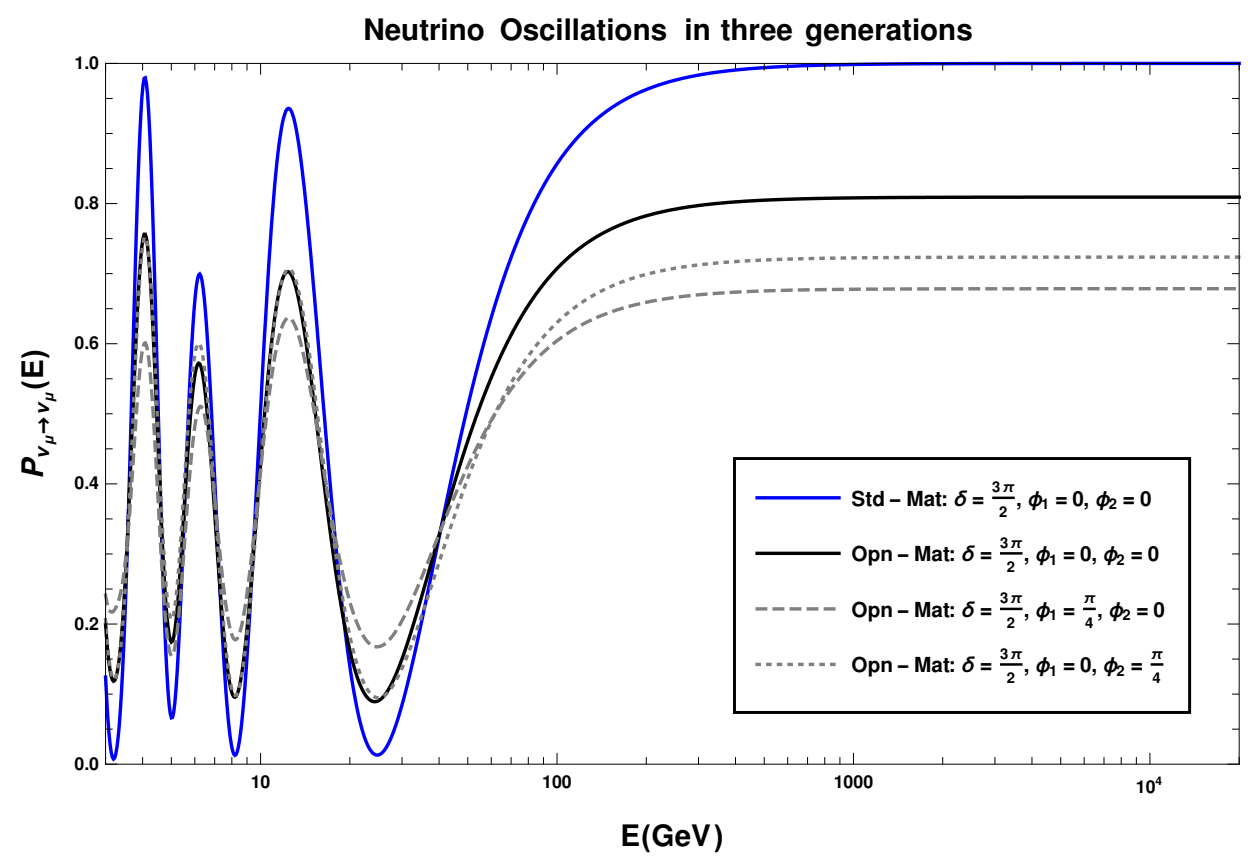

Figure 3.10: The $\nu_{\mu} \rightarrow \nu_{\mu}$ oscillation channel keeping fixed the value of the $\delta$ phase and varying $\phi_{1}$ and $\phi_{2}$. The parameters used are summarized in tables 3.4, 3.5 and 3.6. The Opn, Std and Mat abbreviations stand for open, standard and matter, respectively. Only the Open system case where $\phi_{1}$ is different from zero was obtained by considering the Kossakowski matrix $\tilde{a}$ in (3.39).

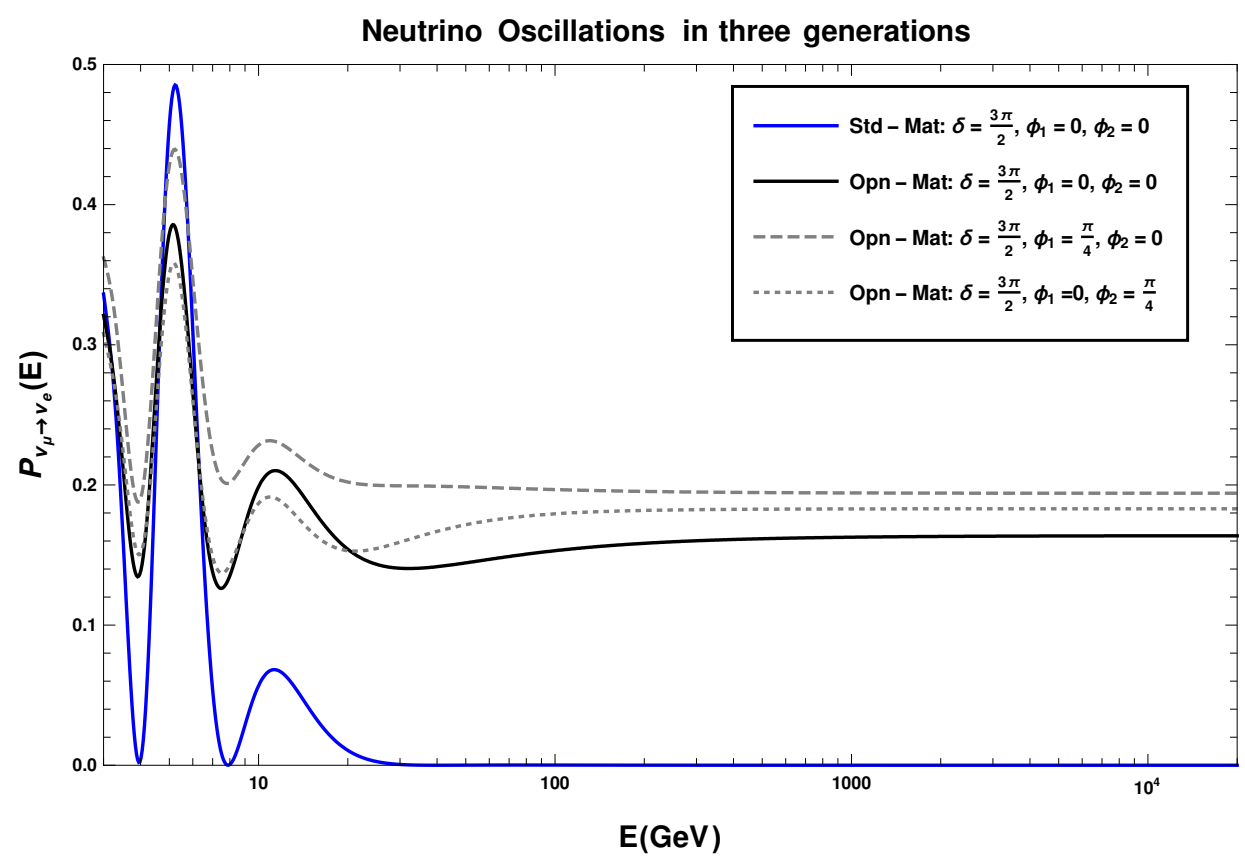

Figure 3.11: Same as figure 3.10, but considering the $\nu_{\mu} \rightarrow \nu_{e}$ oscillation channel. 


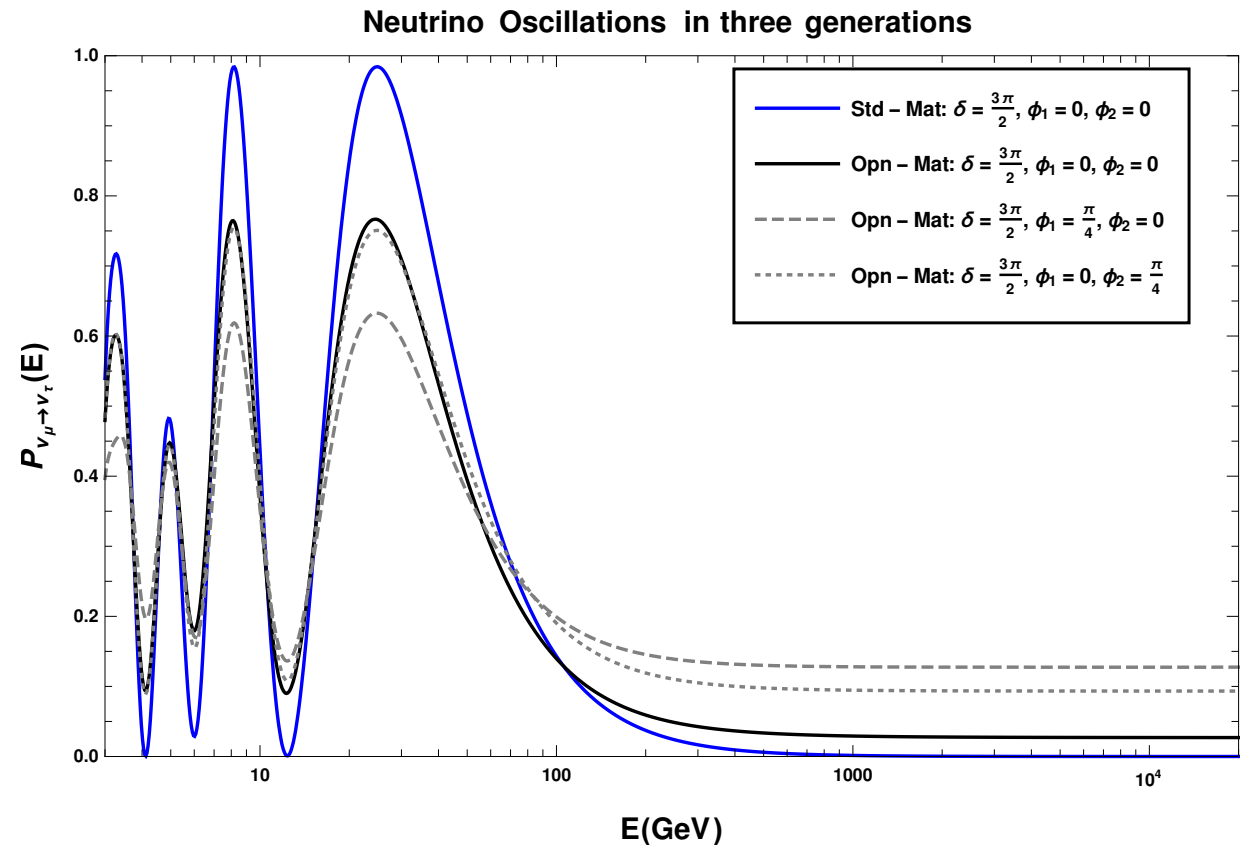

Figure 3.12: Same as figure 3.10, but considering the $\nu_{\mu} \rightarrow \nu_{\tau}$ oscillation channel.

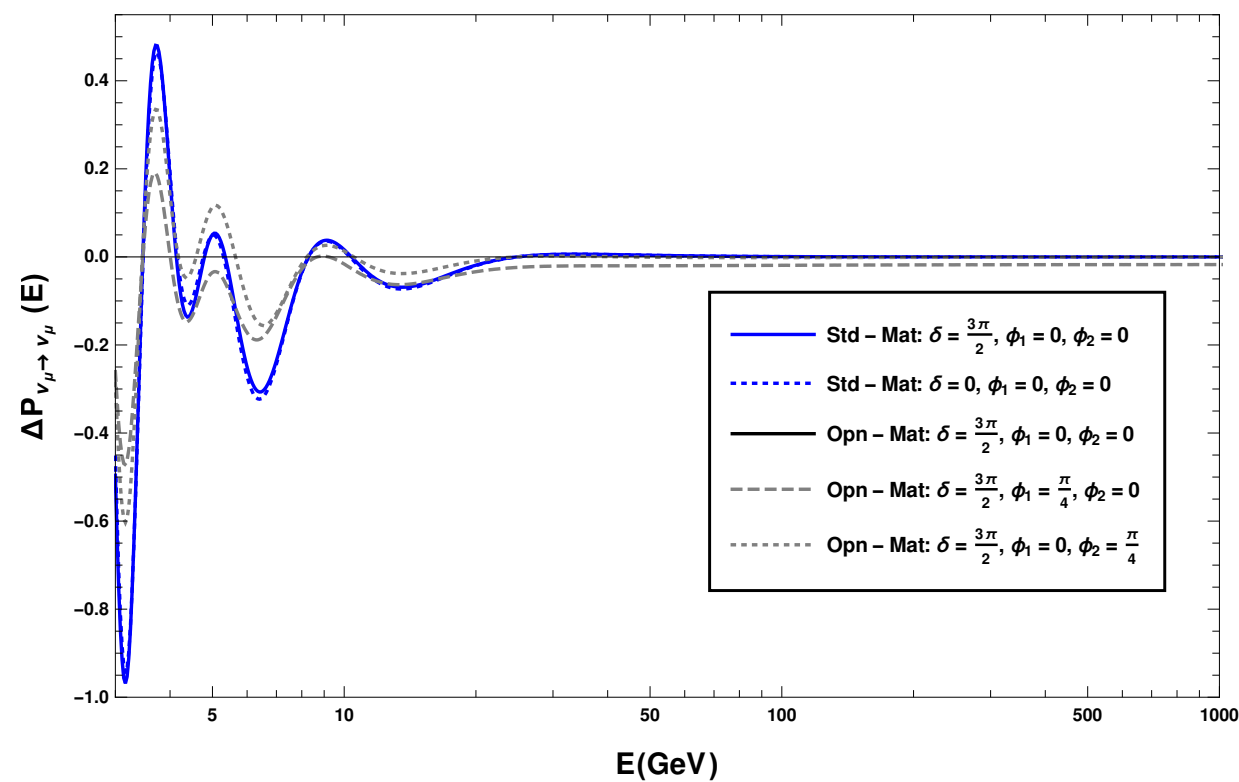

Figure 3.13: The CPT violation in the $\nu_{\mu} \rightarrow \nu_{\mu}$ channel (only CP for the other $\nu_{\mu} \rightarrow \nu_{e}$ and $\nu_{\mu} \rightarrow \nu_{\tau}$ oscillation channels) for different combination of phases. We also show a standard scenario where the CPT $(\mathrm{CP})$ violation is purely due to the matter effects. The parameters used are summarized in tables 3.4, 3.5 and 3.6. The Opn, Std and Mat abbreviations stand for open, standard and matter, respectively. Only the Open system case where $\phi_{1}$ is different from zero was obtained by considering the Kossakowski matrix $\tilde{a}$ in (3.39). 


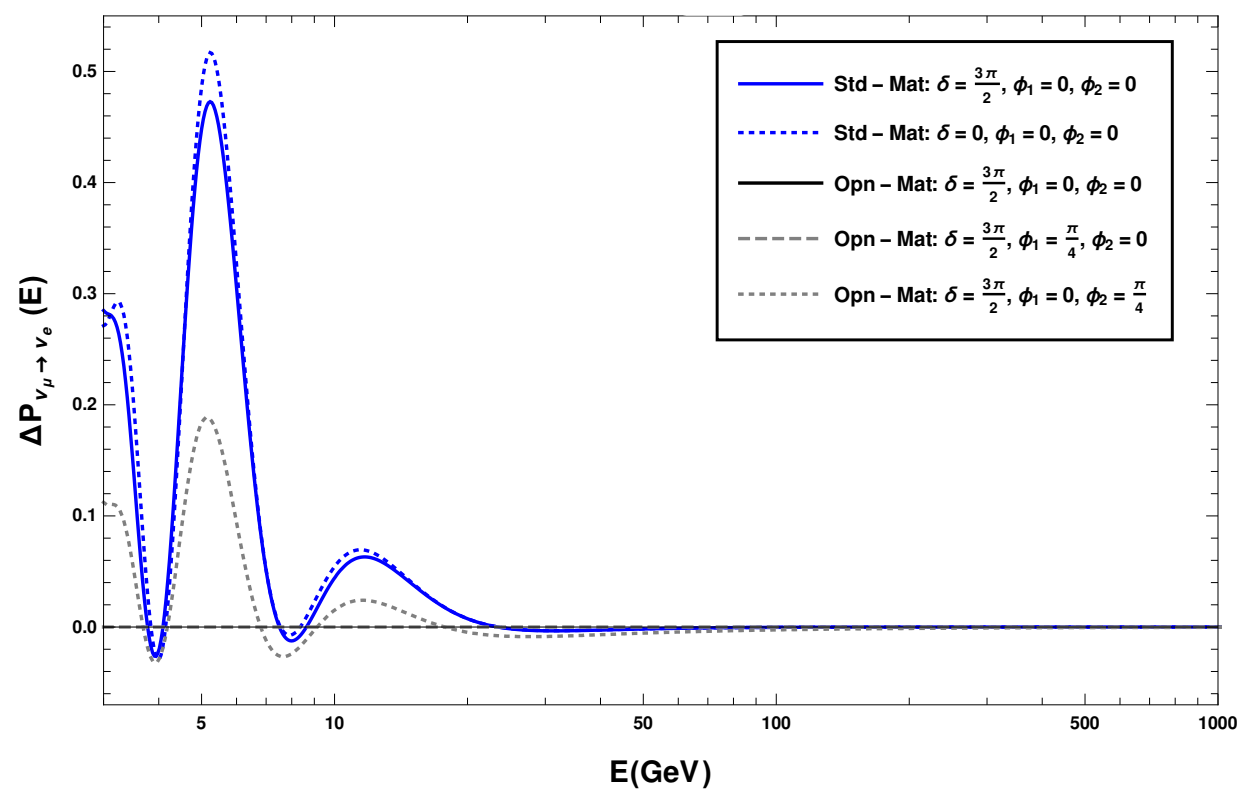

Figure 3.14: Same as figure 3.13, but considering the $\nu_{\mu} \rightarrow \nu_{e}$ channel.

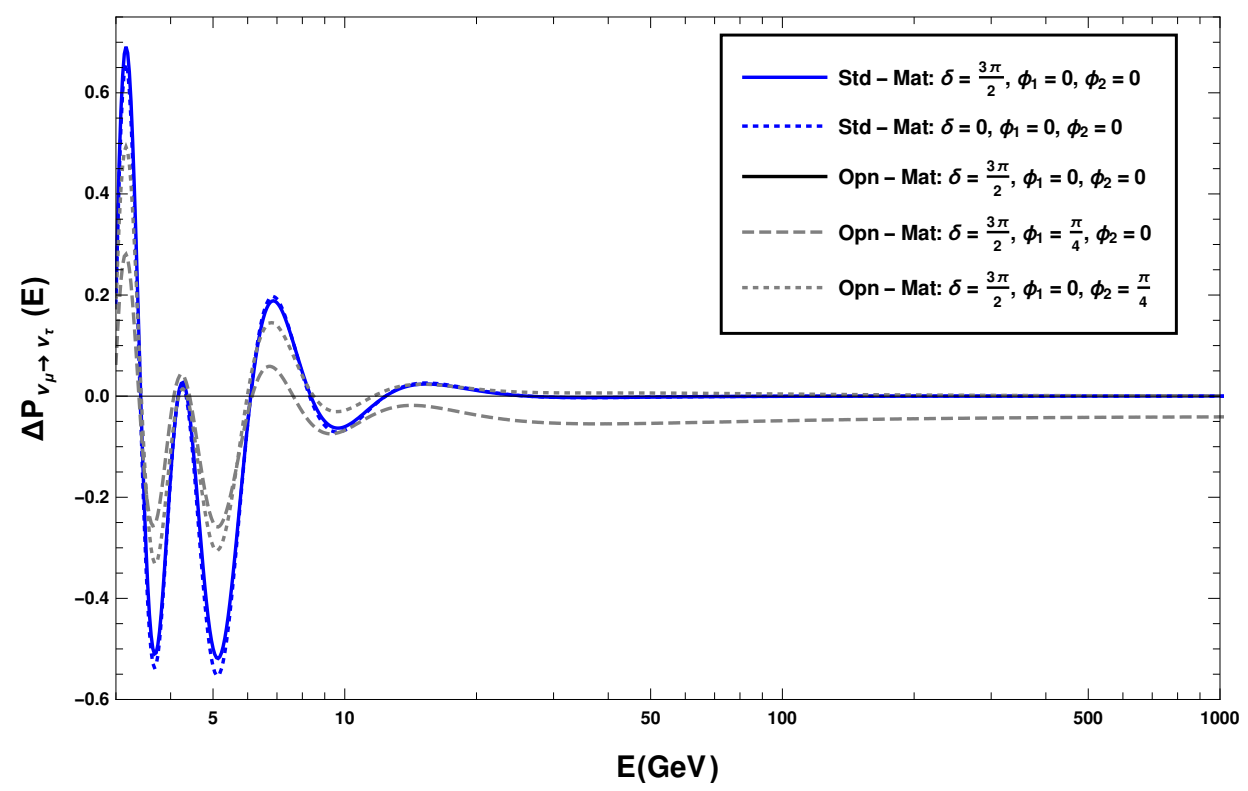

Figure 3.15: Same as figure 3.13, but considering the $\nu_{\mu} \rightarrow \nu_{\tau}$ channel. 


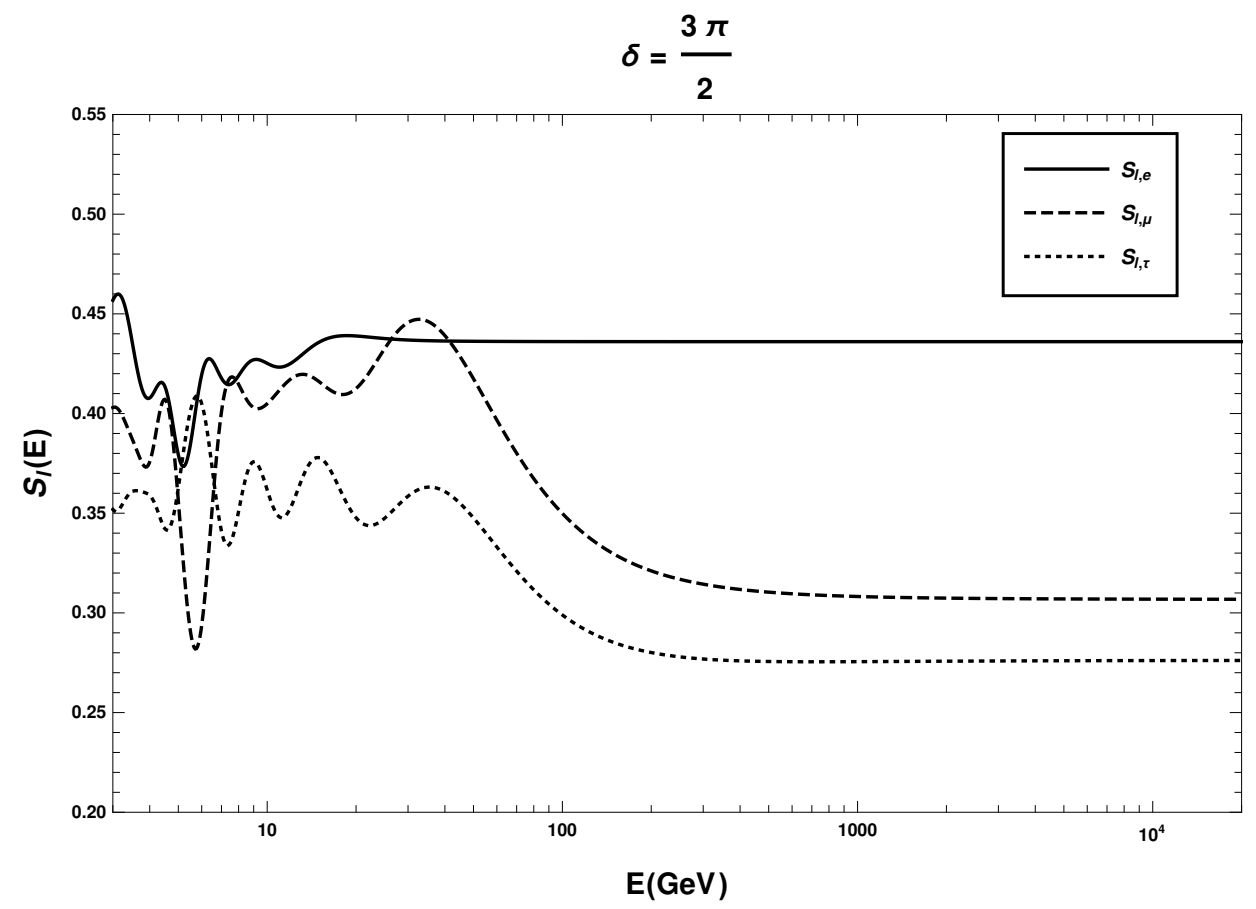

Figure 3.16: Linear entropy for all three neutrino flavor considering only the phase $\delta$ fixed to the value $3 \pi / 2$. The Open System effects were included by using the Kossakowski matrix $a$ in equation (3.39).

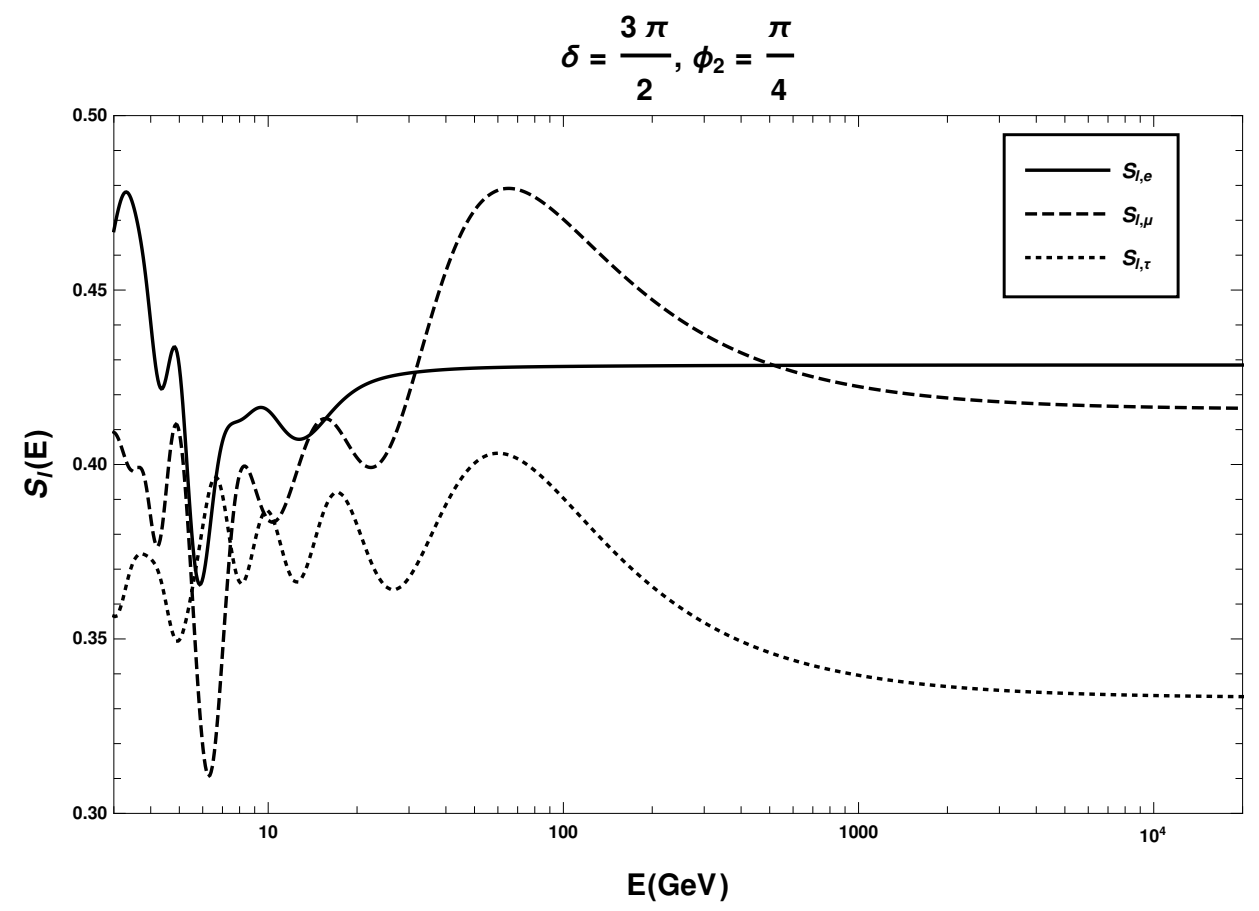

Figure 3.17: Linear entropy for all three neutrino flavor considering both the phases $\delta$ and $\phi_{2}$ fixed to values $3 \pi / 2$ and $\pi / 4$, respectively. The Open System effects were included by using the Kossakowski matrix $a$ in equation (3.39) 


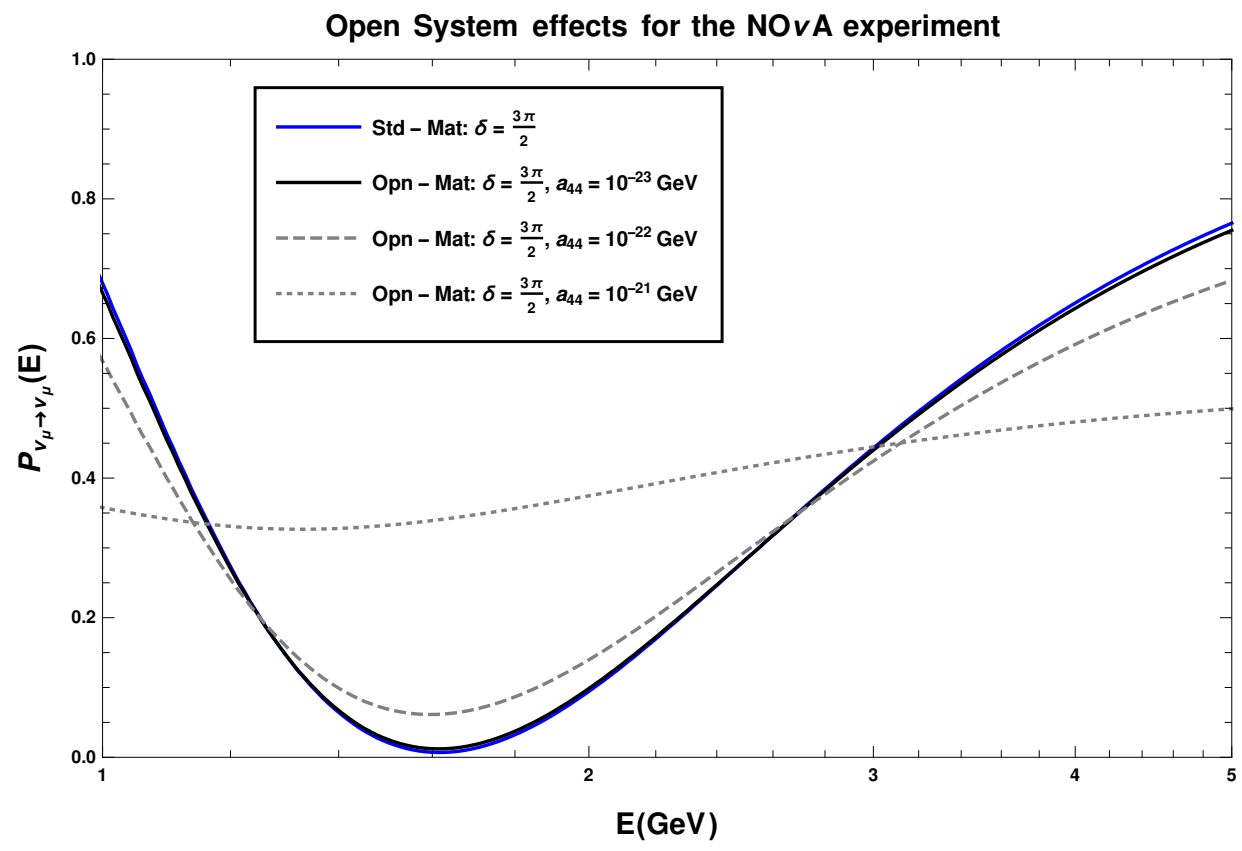

Figure 3.18: Consequences of the Open System interactions using the Kossakoski matrix $a$ in equation (3.39) for the energy range in the reach of the $\mathrm{NO} \nu \mathrm{A}$ experiment. The baseline distance is $L_{\mathrm{NO} \nu \mathrm{A}}=810 \mathrm{~km}$ and the average density the neutrino faces before reaching the detector is $\rho_{e}=2.84 \mathrm{~g} / \mathrm{cm}^{3}$ or $n_{e}=6.53 \times 10^{-18} \mathrm{GeV}^{3}$ in the same units of table 3.4. 


\subsection{Conclusions}

This chapter serves as a bridge connecting the abstract description of the Open Quantum Systems introduced in chapter [2] and neutrino oscillations discussed in chapter [1]. We saw that two generations is a good starting point because of its simplicity, however, even in this scenario, we were able to see interesting consequences of the Open System effects. Furthermore, we have introduced the Checker map (3.1) which encapsulates in a succinct form the relation between the Kossakowski matrix and the Dissipator.

After that we moved to the discussion of the more realistic description considering three generations in matter. By using parameters at the reach of the IceCube experiment we were able to study how the Open System effects would affect the oscillation pattern, motivating the reasons why the IceCube experiment is our choice to search for these effects. We found that an excess of $\nu_{e}$ induced events might be a signal of the presence of Open System interactions.

Besides that, we saw that in the Open System framework we have three important phases, $\delta, \phi_{1}$ and $\phi_{2}$, such that even the Dirac phase $\delta$ affects the oscillation pattern. One direct consequence of this is $\mathrm{CP}$ violation. We have shown that even though matter effects already violate $\mathrm{CP}$, the changes at high energy are solely due to the Open System effects. Furthermore, the fact that $\phi_{1}$ and $\phi_{2}$ are relevant to neutrino oscillations implies that, in principle, we can distinguish between Dirac and Majorana neutrinos.

In order to compare the sensitive of the IceCube with the reach of other experiments, we also exhibited a result using the parameters from the $\mathrm{NO} \nu \mathrm{A}$ experiment. We inferred that indeed the IceCube is a better choice to search for Open System effects.

Now we are ready for the next chapter, where we will study the atmospheric neutrino oscillation with Open System effects in the IceCube experiment. 


\section{CHAPTER 4}

\section{ICECUBE ATMOSPHERIC NEUTRINOS IN AN OPEN SYSTEM APPROACH}

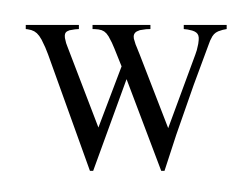

E have seen how the Open System effects can change the oscillation phenomenon at the probability level, but this is not enough to connect the theoretical predictions with the real physical world. In order to move from theoretical grounds to testable predictions we have to compute the number of events expected given an oscillation probability for a certain experimental setup.

In the previous chapter we motivated why IceCube is an appealing choice. Located at the Geographic South Pole, the experiment was primarily designed for the study of neutrinos produced in astrophysical sources by measuring the end products of the possible interactions of these neutrinos with the Antarctic ice. However, the reach of the experiment is not restricted only to the astrophysical neutrinos, inside the IceCube detector there is an area where the experimental setup is densely displayed in order to lower the energy threshold to a value compatible with the study of atmospheric neutrino oscillations. This denser region is called the DeepCore inner detector.

In this work we will concentrate on the atmospheric neutrino data collected by the IceCube collaboration in both the IceCube and DeepCore detectors, covering an energy range between $6 \mathrm{GeV}$ and $20 \mathrm{TeV}$, the same energy range we explored in the previous chapter. For this purpose in the first section we briefly describe the IceCube experiment and the particularities of the data sets we are considering.

After that, in the second section, we will discuss the basic features of atmospheric neutrinos, this is important not only to understand better what are these neutrinos but also to settle a 
IceCube atmospheric neutrinos in an Open System approach

few important details concerning our simulation. In the third section we will exhibit the model we used to calculate the neutrino oscillation probability for the IceCube experiment, it is a generalization of what we did in chapter [3] for a variable baseline length. Next, in section four, we will discuss the details of the statistical analysis we performed to fit the data and properly interpret our results and then, in section five, we will present and discuss our results obtained by employing all the machinery we have developed in this work.

Finally, in the last section, we will comment on previous works in the literature related to the Open System effects in neutrino oscillations. This is important since we have approached the subject in a manner somewhat different than most of these works.

\subsection{The IceCube experiment}

Designed to detect high energy $(E>100 \mathrm{GeV})$ neutrinos coming from the most violent astrophysical events in the universe, the IceCube experimental setup consists of 86 strings of detectors embedded into boreholes that reach $2450 \mathrm{~m}$ depth in the South Pole glacial ice. The instrumented region starts at $1450 \mathrm{~m}$ depth and is arrayed over a cubic kilometer of ice in a hexagonal shape, 60 digital optical modules (DOMs) that are responsible for the signal detection are attached to each string. In the IceCube detector the strings are $125 \mathrm{~m}$ apart while the distance between DOMs is $17 \mathrm{~m}$. However, inside the IceCube detector exists an inner region, the DeepCore sub-detector, where strings are only $70 \mathrm{~m}$ apart, the DOMs have higher quantum efficiency and are separated only by $7 \mathrm{~m}$. The DeepCore detector setup allows to lower the energy threshold from $E \approx 100 \mathrm{GeV}$ to $E \approx 5 \mathrm{GeV}$ permitting the IceCube experiment also to study atmospheric neutrino oscillations with a $L / E$ ratio comparable to long-baseline experiments [91]. The energy range that IceCube can detect neutrinos is larger than the reach of other experiments which allows the collaboration to study not only possible new physics scenarios but also to provide complementary tests as IceCube can measure neutrino oscillation parameters.

The IceCube experiment can distinguish between two kinds of events topologies:

1. Cascade-like events, which are produced by neutral current interaction of all neutrino flavors, charged current interactions of $\nu_{e}$ and by the majority of charged current $\nu_{\tau}$ interactions, resulting in an electromagnetic or hadronic cascade that leaves a signal in the detector of a few meters for atmospheric neutrinos.

2. Track-like events produced through $\nu_{\mu}$ charged current interactions resulting in a hadronic shower at the interaction vertex and a muon which can travel large distances in the detector. A $\nu_{\tau}$ can also produce a track-like event due to the subsequent $\tau$ decay into a $\mu$, but 


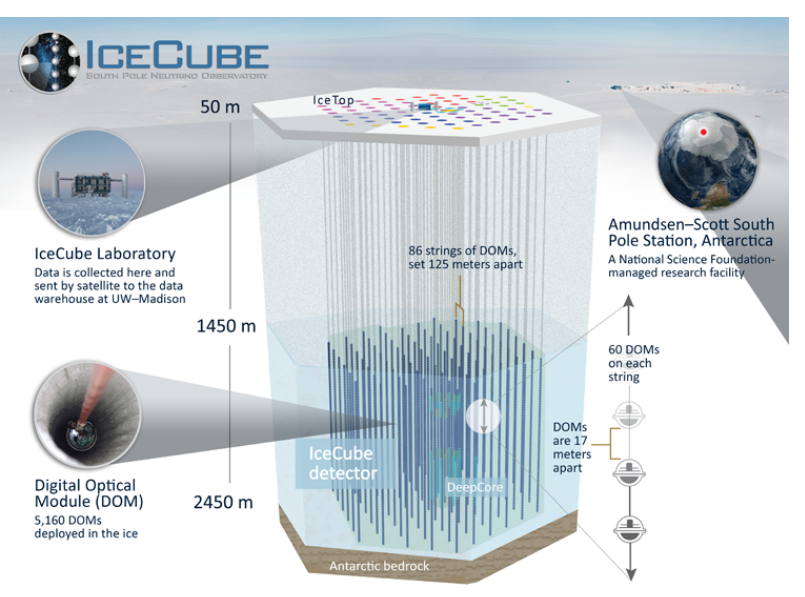

(a) The IceCube detector. The instrumented region starts at $1450 \mathrm{~m}$ depth and extends over $1 \mathrm{~km}$. The denser inner region, known as DeepCore, has a lower energy threshold which makes the study of atmospheric neutrino oscillations feasible. Figure taken from IceCube webpage.

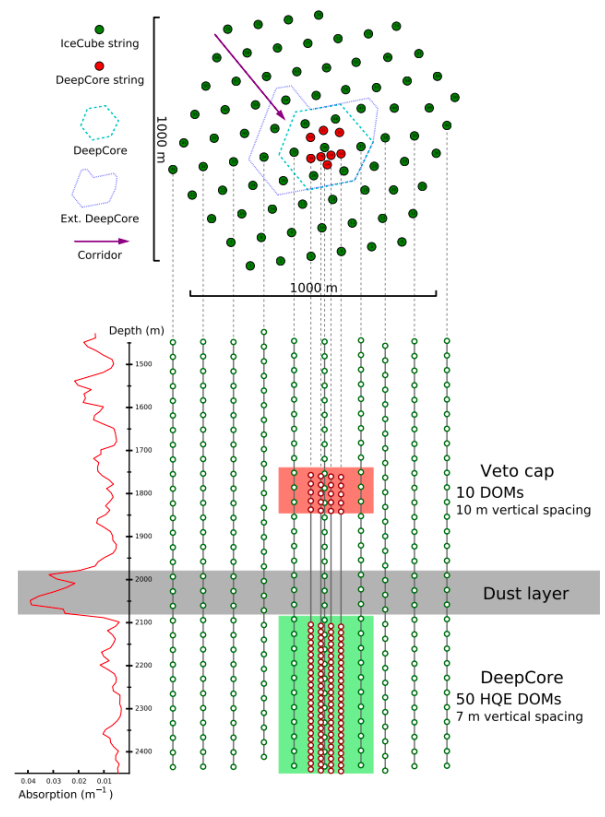

(b) The instrumented region of the IceCube experiment, emphasizing the separation between the IceCube and DeepCore detectors. Figure taken from [92].

Figure 4.1: The IceCube experiment.

for energies below $E<100 \mathrm{GeV}$ the resultant $\mu$ is not visible and this signature cannot be distinguished from cascade-like events [93].

The secondary particles, produced by the primary neutrino interactions, can travel faster than the light in ice, generating Cherenkov radiation which is used to select the event, classify its topology and reconstruct the information about the neutrino.

In this work we will use two different public data sets ${ }^{1}$ released by the IceCube collaboration, whose related works can be found in [92, 94]. First, let us start by discussing [92], in this work the collaboration use the data taken between the operating period of April 2012 to May 2015 to measure the atmospheric tau neutrino appearance with the DeepCore inner detector. The report used two distinct, but not independent, samples, labelled analysis $\mathcal{A}$ and $\mathcal{B}$, the difference between then being the event selection and analysis methods employed. Since analysis $\mathcal{A}$ is the main result of the collaboration and encompass a bigger number of events, we will restrict the discussion only to this sample, the details concerning analysis $\mathcal{B}$ can be found in $[92,95,93]$.

\footnotetext{
${ }^{1}$ Available online on https://icecube.wisc.edu/science/data.
} 
IceCube atmospheric neutrinos in an Open System approach

Covering a livetime of 1006 days the data released in sample $\mathcal{A}$ is binned into a $8 \times 10 \times 2$ dimensional histogram in the variables reconstructed energy $E_{\nu}^{\text {reco }}$, reconstructed cosine of the zenith angle $\cos \theta_{z}^{\text {reco }}$, and Particle IDentifier (PID), respectively. The energy is logarithmically spaced between $5.6 \mathrm{GeV}$ and $56 \mathrm{GeV}$, while the reconstructed cosine of the zenith angle is linearly spaced between -1 and 1 . The zenith angle orientation is defined in such a way that for $\cos \theta_{z}^{\text {reco }}<0$ the incoming neutrino crossed the Earth before reaching the detector. The PID is used to distinguish between the two possible event topologies, it assumes the value 0 for cascade-like events and 1 for track-like ones. To reduce the background contamination the collaboration uses the strings of the IceCube experiment as a veto region for the DeepCore inner detector, establishes a minimum energy threshold for the events and considers only neutrino interaction vertices happening within the DeepCore volume. For reference, at $E_{\nu}^{\text {reco }}=20 \mathrm{GeV}$, the energy and angular resolution of the experiment is $24 \%(29 \%)$ and $10^{\circ}\left(16^{\circ}\right)$ for track-like (cascade-like) events, respectively [96]. All the nuisance parameters included in the analysis performed by the collaboration can be found in [95], in table 4.1 we summarized only the detector related nuisance parameters that shall be used in our study in section 4.4. The best fit values were obtained by the measurement of $\nu_{\tau}$ appearance with DeepCore [92].

\begin{tabular}{|c|c|c|}
\hline Parameter & Prior & Best fit CC + NC [only CC] \\
\hline Optical Eff., Overall (\%) & $100 \pm 10$ & $98.4[98.4]$ \\
\hline Optical Eff., Lateral $(\sigma)$ & $0.0 \pm 1.0$ & $0.49[0.48]$ \\
\hline Optical Eff., Head-on (a.u) & - & $-0.63[-0.64]$ \\
\hline Bulk Ice, Scattering (\%) & $100 \pm 10$ & $103.0[102.8]$ \\
\hline Bulk Ice, Absorption (\%) & $100 \pm 10$ & $101.5[101.7]$ \\
\hline
\end{tabular}

Table 4.1: Nuisance parameters along with their associated priors when applicable and the best fit values from analysis $\mathcal{A}$ when fitting the charged and neutral current $\nu_{\tau}$ normalization combined $(\mathrm{CC}+\mathrm{NC})$ and the charged current alone $[\mathrm{CC}]$. Priors are given as central value together with the $\pm 1 \sigma$ ranges when a Gaussian prior is imposed. Table taken from [92], modified to include only the detector related nuisance parameters.

In addition to the data sample, the report also includes one Monte Carlo file for atmospheric neutrino simulated events and one containing atmospheric muon simulated events. In the first the simulated events are classified by the variables true energy $E_{\nu}^{\text {true }}$, true cosine of the zenith angle $\cos \theta_{z}^{\text {true }}$, reconstructed energy $E_{\nu}^{\text {reco }}$, reconstructed consine of the zenith angle $\cos \theta_{z}^{\text {reco }}$, PID, weight per event, PDG code and type. The true variables are the simulated ones, the weight is a crucial variable in the analysis as it takes into account all the detection variables such as the effective area, cross section and differences due to the event topology. It has to be multiplied by the atmospheric flux and the livetime of the data taken period considered to compute 
the event contribution to the correspondent bin. Finally, the PDG code is the standard Particle Data Group identification of particles [97] and the type variable is used to classify the type of the simulated interaction. Furthermore, how to correct the weight considering the impact from detector uncertainties (see table 4.1) is encoded on the files named hyperplanes and the usage is explained in the included python jupyter notebook.

Now moving to the discussion of the other data sample. In work [94] we can find the description of the report, labelled analysis IC86. The data sample released in IC86 covers the period between the years 2011 and 2012 with a livetime of 343.7 days. The data is displayed in a $10 \times 21$ histogram in the variables reconstructed energy, covering the range $E_{\nu}^{\text {reco }} \in(400 \mathrm{GeV}, 20 \mathrm{TeV})$ and cosine of the zenith angle, linearly spaced starting at $\cos \theta_{z}^{\text {reco }}=0.24$ with spacing of 0.06 , respectively. The zenith angle resolution is in the range $\sigma_{\cos \theta_{z}} \in[0.005,0.015]$ while the energy resolution is $\sigma_{\log _{10}\left(E_{\mu} / \mathrm{GeV}\right)} \sim 0.5$.

Together with the data sample there is also the Monte Carlo file, displayed in roughly the same variables as in analysis $\mathcal{A}$ discussed before. The only difference is the addition of two columns, one related to the neutrino flux from pions and the other related to the neutrino flux from kaons. Furthermore, in this analysis the Monte Carlo weights are already corrected for the nominal values of detector uncertainties, since IC86 was used to the study of sterile neutrinos there is no best fit of the detector uncertainties available. Finally, the last important detail concerning this sample is that for this energy range most of the observed events $(\sim 99 \%)$ are basically induced only by muon neutrinos, this will be important for the number of event computation (see section 4.4).

\subsection{Atmospheric neutrinos}

The fluxes of different ionizing particles that reach the Earth, composed mainly of protons considering the energy spectrum above $10^{10} \mathrm{eV}$, are collectively called Cosmic Rays. These particles can interact with the air molecules in the atmosphere through electromagnetic and strong interactions producing charged mesons which decay into neutrinos and charged leptons (see figure 4.2). The joint contribution of the neutrinos produced in the mesons and lepton decays is what is called atmospheric neutrinos.

The atmospheric neutrino sector played a crucial role in the discovery of the oscillation phenomenon and remains as one of the important sources of information about the neutrino physics [16, 17, 18, 21, 19, 20, 22].

The energy spectrum $\phi_{\nu}$ of the neutrinos produced in the primarily Cosmic Rays interactions is given as a first approximation by a power law as $\frac{\mathrm{d} \phi_{\nu}}{\mathrm{d} E_{\nu}} \propto E_{\nu}^{-\gamma}$, where $\gamma$ is called the spectral 


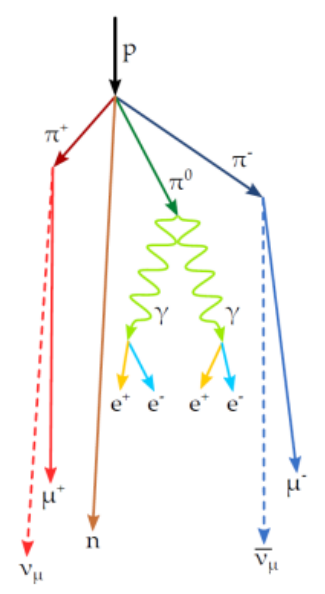

Figure 4.2: The pion meson production by a Cosmic Ray proton and the subsequent decay into charged leptons and neutrinos or antineutrinos depending on the lepton produced. Figure taken from [93].

index, it is an energy dependent variable which varies according to the origin of the Cosmic Ray. This approximation is enough for didactic purposes, but a more advanced calculation [98] is necessary in order to make reliable predictions for atmospheric neutrinos.

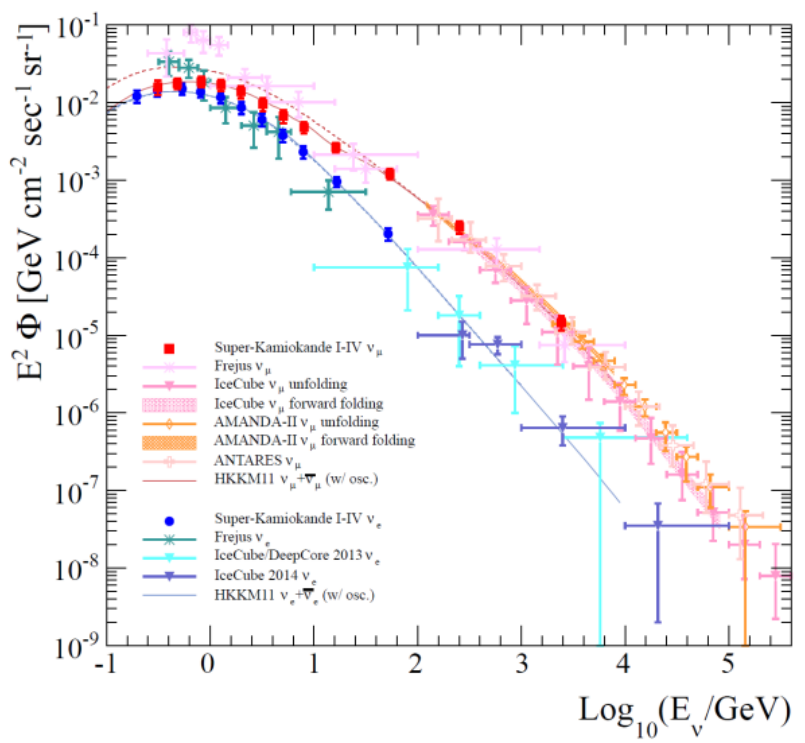

Figure 4.3: Energy spectrum of the atmospheric electron and muon neutrino flux as predicted by the HKKM research group [98]. Figure taken from [93].

For this purpose, in this work we have used the nominal atmospheric neutrino and antineutrino fluxes predictions from [99] for the South Pole, shown in figure 4.4. Note that $\nu_{\tau}$ is not 


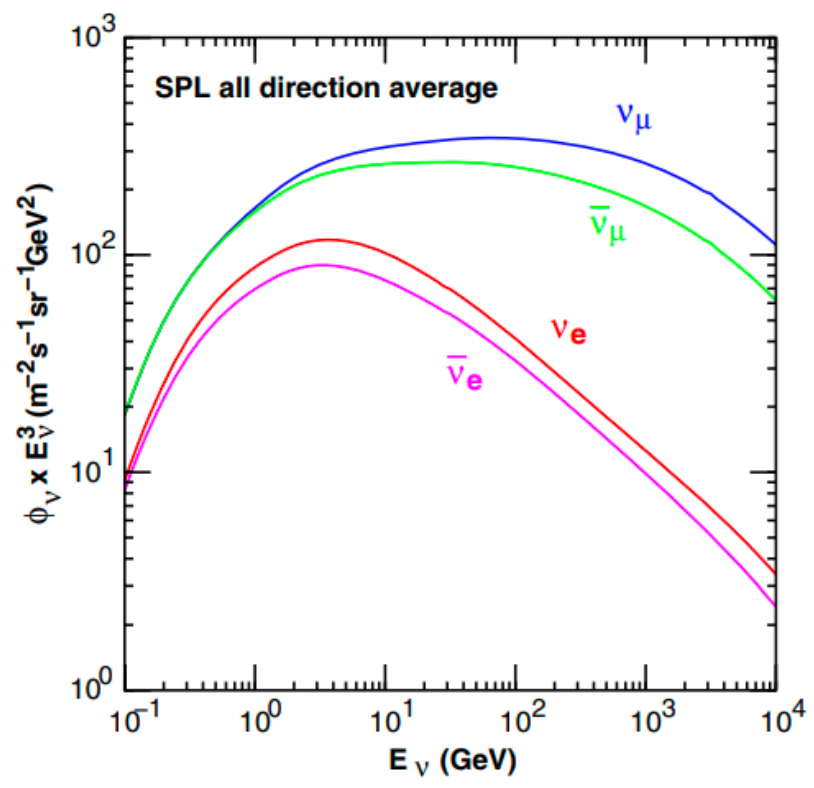

Figure 4.4: Energy spectrum of the atmospheric neutrinos and antineutrinos $\nu_{\mu}, \nu_{e}, \bar{\nu}_{\mu}$ and $\bar{\nu}_{e}$ averaged over all directions for the Geographic South Pole. Figure taken from [99].

present since it is not generated in pion or kaon decays, the relevant mesons for the energy range considered in our work. Even if we considered the $\nu_{\tau}$ flux produced by the heavy meson decays (denominated prompt contribution), it is an order of magnitude smaller than the contribution of the other two neutrino flavors [93].

With the aid of the IceCube Monte Carlo files and in possession of the neutrino and antineutrino atmospheric fluxes the only remaining ingredient to complete our analysis is the oscillation probability.

\subsection{The $\mathbf{N}$ layer model}

The $N$ layer model consists of slicing the Earth into layers of constant matter density, it is an extension of equations (3.37) and (3.38) for a variable baseline length. Suppose that as the neutrino travelled its path inside the Earth, it had crossed $N$ layers of matter ${ }^{2}$, each with constant density $n_{i},(i=1, \ldots, N)$, and that the distance travelled in each layer is $L_{i}\left(\theta_{z}\right)^{3}$. Not only the distance but also the number of layers the neutrino crosses before reaching the detector depends on the zenith angle $\theta_{z}$ where the neutrino was produced. This variable is measured in

\footnotetext{
${ }^{2}$ Since the matter number density $n_{e}$ is always the electron number density we will omit the index $e$ from now on.

${ }^{3}$ How $L_{i}\left(\theta_{z}\right)$ depends on the zenith angle is shown in appendix D.
} 
relation to the IceCube experiment and is equal to $\pi$ for a neutrino crossing the Earth diameter (see figure 4.5).

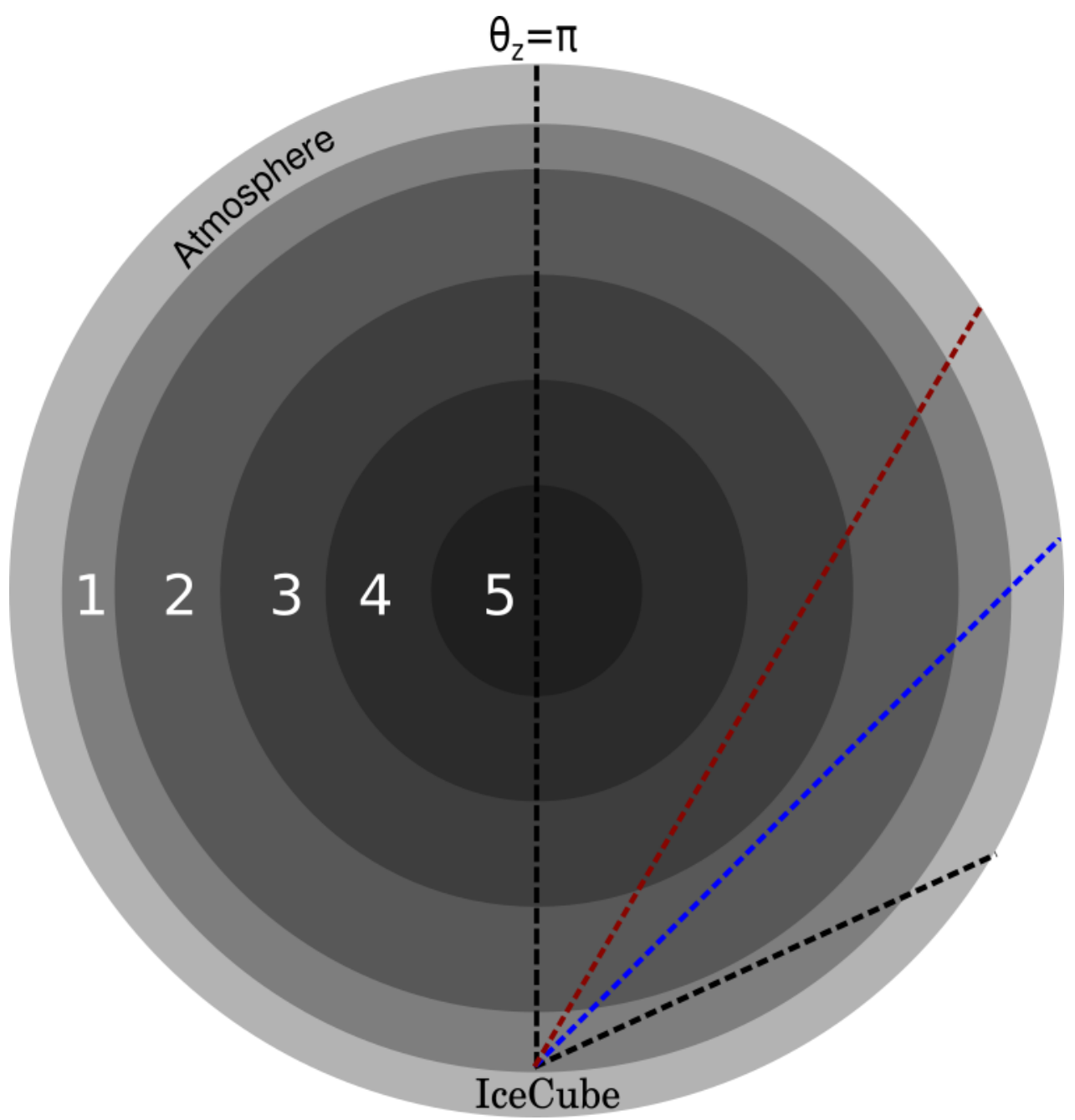

Figure 4.5: Different possible paths that a neutrino can travel through Earth before reaching the IceCube detector. We defined $\theta_{z}=\pi$ for a neutrino which crosses the full Earth diameter

From equation (3.36) we know how the initial flavor basis are obtained from the mass basis

$$
\begin{aligned}
& \rho_{e}(0)=U^{\dagger} \rho_{1}(0) U, \\
& \rho_{\mu}(0)=U^{\dagger} \rho_{2}(0) U, \\
& \rho_{\tau}(0)=U^{\dagger} \rho_{3}(0) U .
\end{aligned}
$$

Furthermore, modifying (3.38) just in order to add the layer number dependence, we have that the neutrino exits the first layer after travelling the distance $L_{1}\left(\theta_{z}\right)$ such that the evolved 
flavor state $\alpha$ is given by

$$
\rho_{\alpha}\left(L_{1}\left(\theta_{z}\right)\right)=e^{\mathcal{L}_{1} L_{1}\left(\theta_{z}\right)} \rho_{\alpha}(0)
$$

where $\mathcal{L}_{i}=\mathcal{H}_{i}+\mathcal{D}$, is the sum of the vectorized form of the commutator piece in the $i$ th layer $-i\left[H_{i}, \rho_{\alpha}\left(L_{i}\right)\right]$, considering the matter Hamiltonian in mass basis (1.47) and the threedimensional Dissipator $\mathcal{D}$ (3.35).

The evolution through the remaining layers can be done repeating the same steps, the only difference being the initial state, which is given by the state that has just left the previous layer, such that

$$
\rho_{\alpha}\left(L_{i}\left(\theta_{z}\right)\right)=e^{\mathcal{L}_{i} L_{i}\left(\theta_{z}\right)} \rho_{\alpha}\left(L_{i-1}\left(\theta_{z}\right)\right)
$$

This can be iterated $N$ times and after reaching the last layer the oscillation probability can be calculated by

$$
\mathcal{P}_{\alpha \rightarrow \beta}\left(E, L_{N}\left(\theta_{z}\right)\right)=\operatorname{Tr}\left\{\rho_{\alpha}(0) \rho_{\beta}\left(L_{N}\left(\theta_{z}\right)\right\}\right.
$$

The above result is not restricted to matter layers, it can be used directly to include, for example, the atmosphere, just by considering an extra layer with zero matter density and using the appropriate path length (see appendix D).

The number of Earth layers and their corresponding matter number density remains the same as described in table 3.4, while the oscillation parameters are basically the ones described in table 3.5, with the only difference being the phase $\delta$. From now on we will use the NuFIT best fit value $\delta=222 \pi / 180$.

\subsection{Calculation of atmospheric neutrino events in the Ice- Cube experiment}

With the aid of the Monte Carlo files discussed in section 4.1 the computation of the number of events can be performed by simple steps. First using the $\mathrm{N}$ layer model we can generate probability tables for all the possible neutrino and antineutrino flavors, since IceCube is sensitive to both. Then as the Monte Carlo files contains more than $10^{6}$ simulated events we have enough resolution to obtain a reliable prediction of the number of events by summing the product of the atmospheric neutrino flux, the oscillation probability and the correspondent event weight over all the rows of the Monte Carlo files. As an example, the number of $\alpha$ flavored neutrino and 
IceCube atmospheric neutrinos in an Open System approach

antineutrino events in the reconstructed energy bin defined by energies $E_{\nu, 1}^{\mathrm{reco}}$ and $E_{\nu, 2}^{\mathrm{reco}}$ is given by

$$
\begin{aligned}
N_{\text {events }}^{\alpha}\left[E_{\nu, 2}^{\mathrm{reco}}, E_{\nu, 1}^{\mathrm{reco}}\right] & =T \sum_{ \pm} \sum_{\beta=1}^{3} \sum_{i=1}^{\mathrm{MC} \text { rows }} w^{\alpha}\left(E_{\nu, i}^{\mathrm{reco}}, \theta_{z, i}^{\mathrm{reco}}, E_{i}^{\mathrm{inc}}, \theta_{z, i}^{\text {inc }}\right) \\
& \times \frac{\mathrm{d} \phi_{ \pm, \beta}\left(E_{\nu, i}^{\mathrm{inc}}, \theta_{z, i}^{\text {inc }}\right)}{\mathrm{d} E_{\nu, i}^{\text {inc }} \operatorname{d} \cos \theta_{\mathrm{z}, \mathrm{i}}^{\text {inc }}} P_{\nu_{\beta} \rightarrow \nu_{\alpha}}^{ \pm}\left(E_{\nu, i}^{\text {inc }}, \theta_{z, i}^{\text {inc }}\right),
\end{aligned}
$$

where the variables $E_{\nu}^{\text {inc }}$ and $\theta_{z}^{\text {inc }}$ are the atmospheric neutrino incident energy and zenith angle, respectively. The value of MC rows is given by the total number of rows in the Monte Carlo sample released by the IceCube collaboration, the sum over \pm is present to emphasize the need to sum over $\alpha$ flavored neutrinos + and antineutrinos - , since IceCube can measure both indistinguishable. The variable $\phi\left(E_{\nu}, \theta_{z}\right)$ represents the atmospheric neutrino spectrum and, as discussed in section 4.2 we have used the nominal values from [99]. The oscillation probability $P_{\nu_{\beta} \rightarrow \nu_{\alpha}}(E, \theta)$ is obtained using the $\mathrm{N}$ layer model from section 4.3 , and finally, $w^{\alpha}$ is the corresponding event weight. As discussed in section 4.1 this variable must be multiplied by the data taken livetime $T$ and corrected in order to take into account the systematic effects of the detector ${ }^{4}$.

From sections 4.1 and 4.2 we infer that when working with the data set used in [92] we have to consider in equation (4.5) all the possible $\nu_{\mu}$ and $\nu_{e}$ oscillation channels, in such way that $\beta=e, \mu, \tau$. Furthermore, for the energy regime covered in this data set, in addition to the neutrino induced events we have also to add to (4.5) a term $N_{\text {events }}^{\mu}$ to take into account the muon events that might be misidentified as neutrino events. This can be easily done by using the data driven Monte Carlo simulation of the muon events available with the data release.

On the other hand, for the data set used in [94], only the $\nu_{\mu} \rightarrow \nu_{\mu}$ oscillation channel for neutrinos and antineutrinos must be considered, and no extra term has to be included, since for the energy range covered in this data set an energetic muon entering the detector can be distinguished from the neutrino and antineutrino events.

Using equation (4.5) for all the available bins we were able to simulate the atmospheric neutrino events in the IceCube and DeepCore detectors.

\footnotetext{
${ }^{4}$ See section 4.1 for the details of the weight variable for each data set.
} 


\subsection{Statistical analysis}

In order to compare the predictions of the Open System models with the current observed data, we evaluated the goodness of fit of each model by considering a $\chi^{2}$ test statistics, which is defined by

$$
\chi^{2}=\sum_{i=1}^{\text {bins }} \frac{\left(N_{\mathrm{o}, \mathrm{i}}-N_{\mathrm{p}, \mathrm{i}}(\vec{\mu})\right)^{2}}{N_{\mathrm{p}, \mathrm{i}}(\vec{\mu})},
$$

where the sum is over all the bins of observed data samples (see section 4.1) and the values of $N_{\mathrm{o}, \mathrm{i}}$ and $N_{\mathrm{p}, \mathrm{i}}(\vec{\mu})$ correspond to the observed and predicted number of events in the ith bin, respectively. The predicted number of events depends on a set of unknown parameters $\vec{\mu}$ which affect the predictions of the model. Since the uncertainties regarding the Cosmic Ray energy spectrum reflect in uncertainties in the atmospheric neutrino simulations, in this work we have allowed the overall normalization of the atmospheric neutrino and muon fluxes to freely float in our analysis, such that $\vec{\mu}$ corresponds to the overall fluxes normalization and the Open System parameters, defined through the Kossakowski matrix. As an illustrative example, suppose that we want to compute the $\chi^{2}$ for the Dissipator originated by the Kossakowski matrix $a$ discussed in section 3.3 of chapter [3], then we would have $\vec{\mu}=\left(a_{44}, \mathcal{N}_{\phi_{\nu}}, \mathcal{N}_{\phi_{\mu}}\right)$, where $\mathcal{N}_{\phi_{\nu}}, \mathcal{N}_{\phi_{\mu}}$ are the neutrino and muon overall flux normalization factor, respectively. Note that the Dissipator is fixed for each model we want to test while the flux normalization is allowed to vary freely during the fit.

Assuming that the observed number of events can be modeled as a random variable that follows a normal distribution with mean and standard deviation given by the number of predicted events, we can establish the equivalence between the $\chi^{2}$ function and the likelihood of the observed data

$$
\chi^{2}=-2 \log \mathcal{L}
$$

where $\mathcal{L}$ is the likelihood function, which measures how good a model fits the data sample given a set of unknown parameters. In this framework the values of $\vec{\mu}$ that better represent the data are given by those that maximize the likelihood function or, equivalently, those that minimize the $\chi^{2}$. The confidence regions for the allowed values of $\vec{\mu}$ are defined by the variation of the value of $\chi^{2}$ under repeated simulated experiments. In order to restrict the allowed region for the parameters $\vec{\mu}$, we considered variations up to $\sqrt{\Delta \chi^{2}}=\sqrt{\chi^{2}-\chi_{\min }^{2}}=2$, which corresponds to roughly $95 \%$ confidence level and where $\chi_{\min }^{2}$ is the minimum value of $\chi^{2}$. 


\subsection{Discussion of the results}

Now we are in position to reproduce the number of events observed by the IceCube collaboration for both data sets described in section 4.1. The results we obtained for the DeepCore and IceCube detectors are shown in figures 4.6 and 4.7, respectively. Our predictions using the N layer model for the standard oscillation scenario and considering the best fit values from NuFIT (red) are in good agreement with the data (black).

For the $\chi^{2}$ analysis we considered the following scenario, motivated by conditions 1),2) and 3 ) from section 2.2.2. These restrictions imply that the off diagonal terms on the Kossakowski matrix are bounded by the diagonal ones, therefore an interesting choice is the matrix $a_{D}$ below

$$
a_{\mathrm{D}}=\left(\begin{array}{cccccccc}
a_{11} & 0 & 0 & 0 & 0 & 0 & 0 & 0 \\
0 & a_{22} & 0 & 0 & 0 & 0 & 0 & 0 \\
0 & 0 & a_{33} & 0 & 0 & 0 & 0 & 0 \\
0 & 0 & 0 & a_{44} & 0 & 0 & 0 & 0 \\
0 & 0 & 0 & 0 & a_{55} & 0 & 0 & 0 \\
0 & 0 & 0 & 0 & 0 & a_{66} & 0 & 0 \\
0 & 0 & 0 & 0 & 0 & 0 & a_{77} & 0 \\
0 & 0 & 0 & 0 & 0 & 0 & 0 & a_{88}
\end{array}\right) .
$$

We considered that all the nonzero $a_{i i}$ elements in $a_{\mathrm{D}}$ have the same value in the range $a_{i i} \in\left(10^{-26}, 10^{-20}\right) \mathrm{GeV}$. In figures 4.8 and 4.9 we can see that our bottom-up approach starting from the Kossakowski matrix can put strigent bounds on the Open System effects. The difference between the allowed region for both energy regimes is small, but the high energy range (see figure 4.9) gives the most restrictive constrain.

In summary, for $a_{D}$ the joint analysis of the two data sets described in section 4.1 implies that, at $95 \%$ confidence level, the $a_{i i}$ must satisfy the following bound

$$
a_{i i} \leq 1.38 \times 10^{-25} \mathrm{GeV}
$$

The loss of sensitivity that can be seen in figure 4.9 is related to the flux normalization free parameters. For big enough values of the Open System parameters the effects are destructive enough to erase the oscillation completely, which leads to constant probabilities that can be absorbed in the flux normalization. Therefore, we cannot distinguish between a bigger flux with smaller probabilities and the converse.

As can be seen, the restrictions directly in the Kossakowski matrix are more transparent since it is always the starting point of the Open System description. This procedure makes 
easier future comparisons even for different experiments and scenarios.
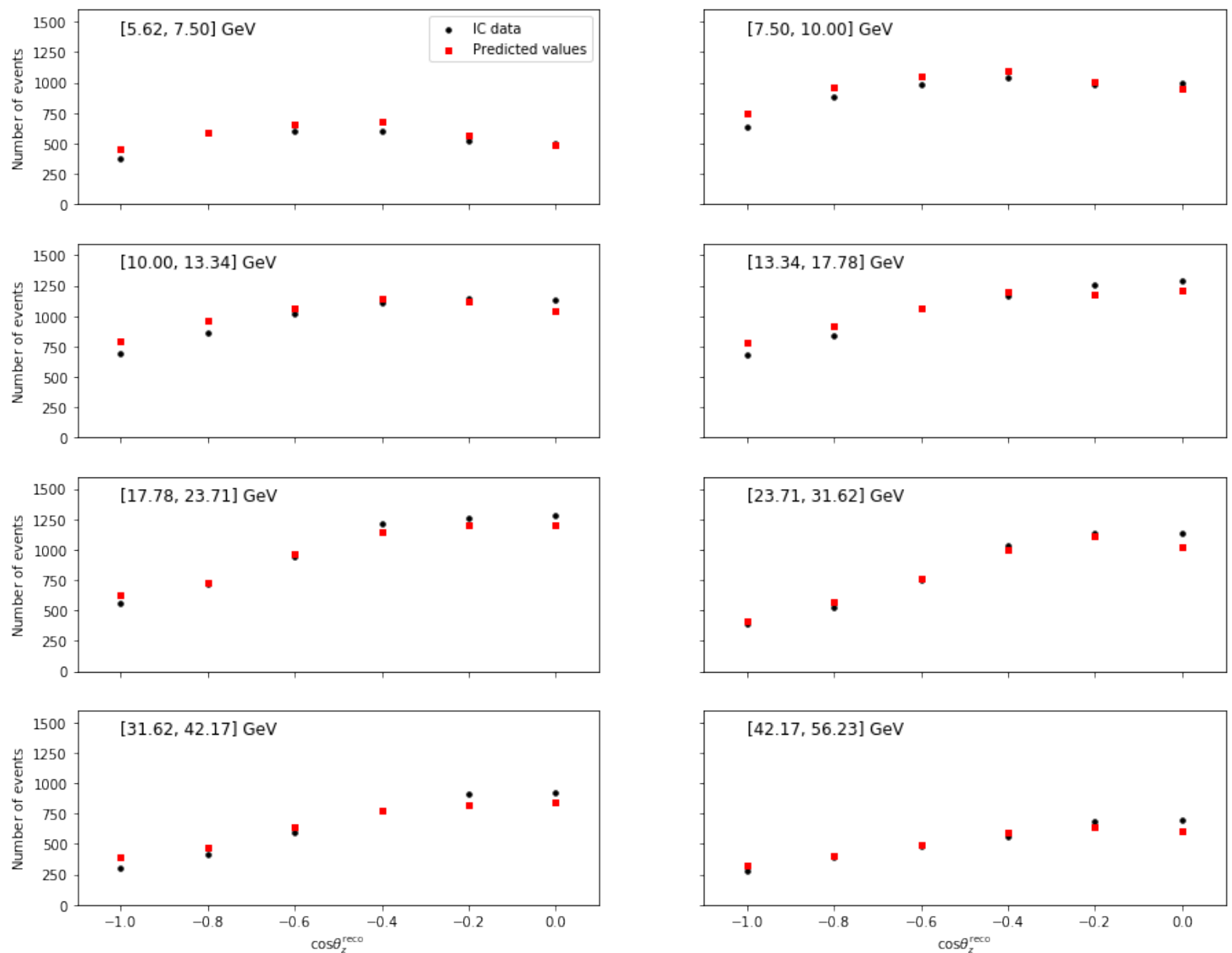

Figure 4.6: Number of events observed (black) and predicted using the $\mathrm{N}$ layer model for standard oscillations (red), considering the data set related to the DeepCore subdetector (see section 4.1). The number of events are shown as a function of $\cos \left(\theta_{z}^{\text {reco }}\right)$ in each reconstructed energy bin. 

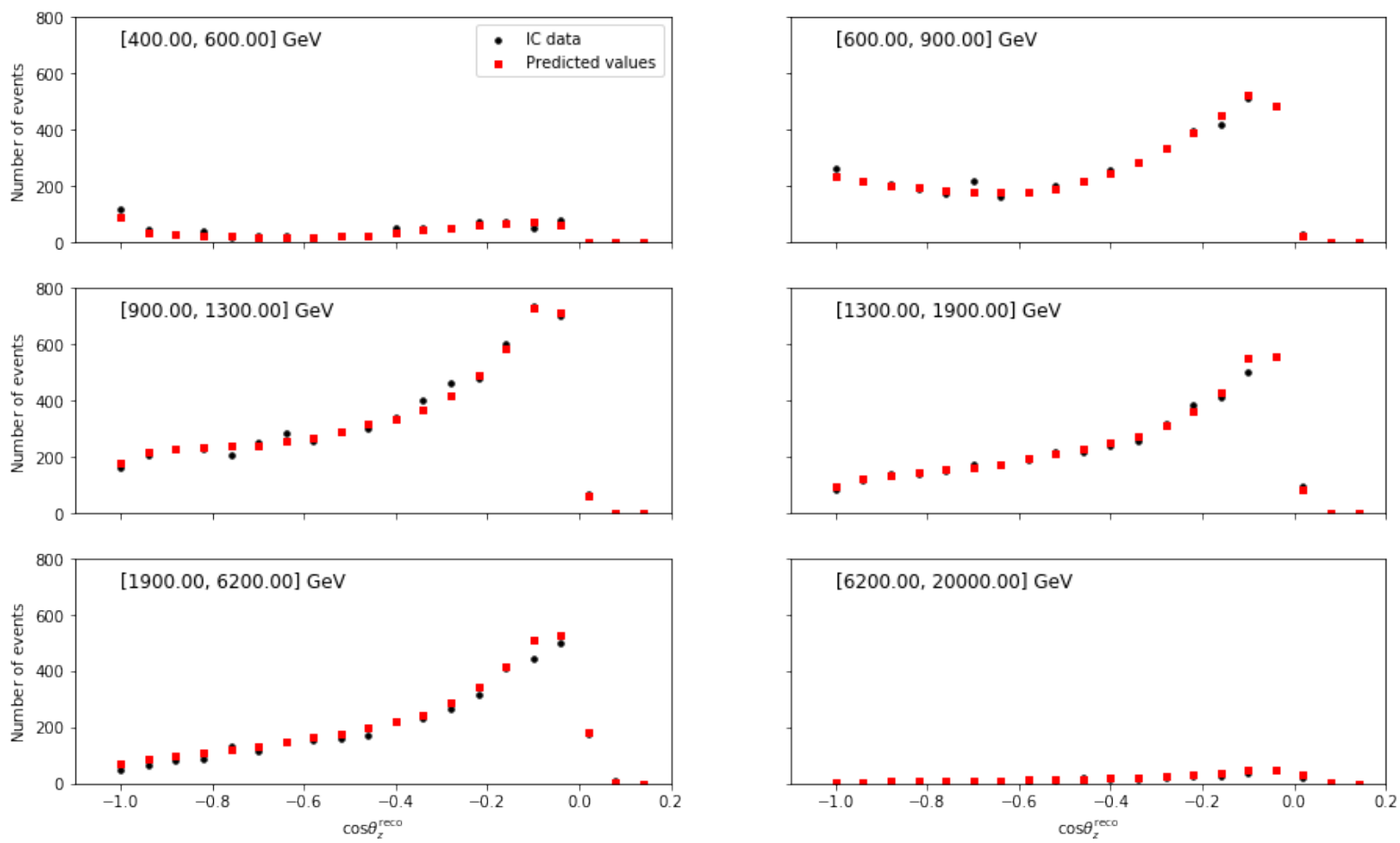

Figure 4.7: Same as figure 4.7 but considering the data set related to the IceCube detector (see section 4.1).

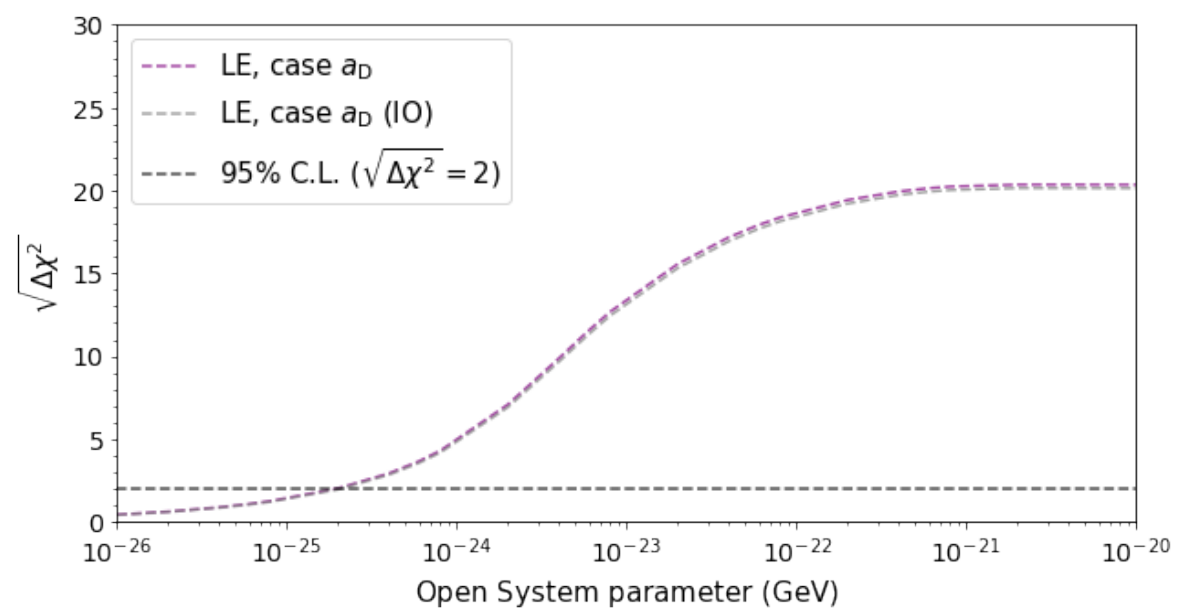

Figure 4.8: $\chi^{2}$ analysis as a function of the Open System parameter, considering the data set related to the DeepCore inner detector (see section 4.1). 


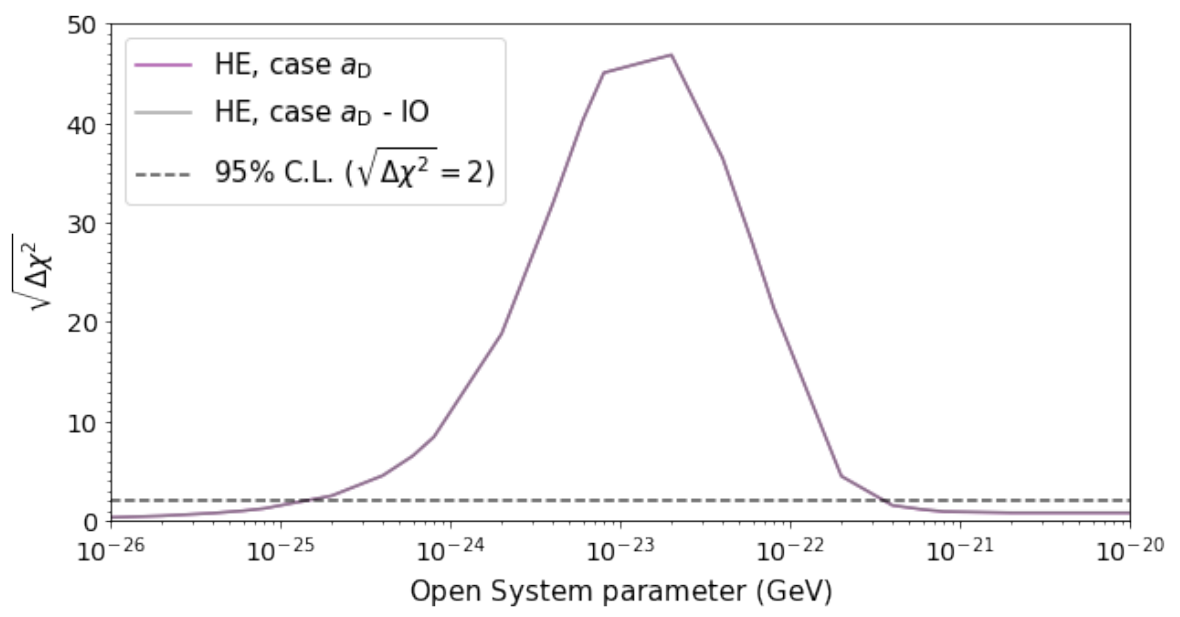

Figure 4.9: Same as 4.8 but considering the data set related to the IceCube detector (see section 4.1). 
IceCube atmospheric neutrinos in an Open System approach

\subsection{Comments of previous works}

In this section we want to discuss previous works that studied the Open System effect in neutrino oscillation to show the differences between the distinct approaches and to emphasize important points concerning the underlying formalism of Open Quantum Systems. In table 4.2 a summary of the works that considered neutrino oscillations with Open Quantum System effects used as a guide to our work is shown. In those the implementation of the Open System effects is done by writing the Dissipator in the following form

$$
\mathcal{D}=\sum_{m}\left(\left\{\rho, D_{m} D_{m}^{\dagger}\right\}-2 D_{m} \rho D_{m}^{\dagger}\right) .
$$

This is analogous to equation (2.35) before decomposing $D_{m}$ in the basis generated by the $F_{i}$ operators. After that, they vectorize equation (4.10) and study directly the effect of those parameters, without the need to use the Kossakowski matrix in any step. Therefore, in this approach the starting point is (3.35). To assure the energy conservation condition they impose that the $D_{m}$ matrix must satisfy

$$
\left[H_{\text {scenario }}^{\text {basis }}, D_{m}\right]=0 \text {. }
$$

In the column "Energy Conservation" of table 4.2 we can see if the authors assumed the energy conservation hypothesis to hold and, if so, on which basis the calculation was performed. The subscript in $H$ indicates if matter effects are present (matter) or not (vacuum), while the superscript indicates in which basis the commutation relation was imposed. If the study considered matter effects, the matter basis is defined as the basis in which the full Hamiltonian is diagonal, while the mass basis is the basis in which the Hamiltonian is given by (1.47).

The works [100, 101, 102, 103, 104, 105, 106] derived bounds on the Open Quantum System parameters of the Dissipator. In general, the authors wrote the only non-zero $\mathcal{D}_{i j}$ elements in (3.35) or in (4.10) as a function a single parameter $\gamma$, which can be or not a function of the energy as we can see from the column " $\gamma$ dependence" in table 4.2. Among these works, in particular, references $[107,108]$, tried to solve the LSND problem by considering Open Quantum System effects. For this purpose, they assumed a very specific energy dependence of the parameter $\gamma$, in such a manner that the modifications decay as the energy increases. Since it is a very particular choice we will not consider them further here.

The remaining works studied the possibility of improving bounds using other experiments, investigated the validity of the formalism used in previous works and studied the Open System effects using perturbative methods.

Reference [85] demonstrated how the definition of the Dissipator directly on the matter basis 
can be problematic.

\begin{tabular}{|c|c|c|c|c|c|}
\hline Ref. & $\gamma$ dependence & Experiment & Generations & Energy Conservation & Date \\
\hline$[103]$ & $\gamma=\gamma_{0}\left(\frac{E}{E_{0}}\right)^{n}$ & KamLAND + Solar & 3 & {$\left[H_{\text {vacuum }}^{\text {flavor }}, D_{m}\right]=0$} & $18 / 07 / 07$ \\
\hline$[108]$ & $\gamma=\frac{\mu^{2}}{E_{\nu}}\left(\frac{40 \mathrm{MeV}}{E_{\nu}}\right)^{r-1}$ & LSND & 3 & {$\left[H_{\text {vacuum }}^{\text {mass }}, D_{m}\right]=0$} & $14 / 05 / 08$ \\
\hline$[106]$ & $\gamma=\gamma_{0}\left(\frac{E}{E_{0}}\right)^{n}$ & MINOS & 2 & Yes/No & $27 / 02 / 14$ \\
\hline$[107]$ & $\gamma=\gamma_{0}\left(\exp \left[-\left(\frac{E}{E_{3}}\right)^{n}\right]-\exp \left[-\left(\frac{E}{E_{1}}\right)^{n}\right]\right)^{2}$ & LSND & 3 & {$\left[H_{\text {vacuum }}^{\text {mass }}, D_{m}\right]=0$} & $26 / 08 / 15$ \\
\hline$[100]$ & $\gamma=\gamma_{0}\left(\frac{E}{E_{0}}\right)^{n}$ & KamLAND & 3 & {$\left[H_{\text {matter }}^{\text {matter }}, D_{m}\right]=0$} & $01 / 04 / 17$ \\
\hline$[109]$ & $\gamma=\gamma_{0}$ & DUNE & 3 & {$\left[H_{\text {matter }}^{\text {matter }}, D_{m}\right]=0$} & $06 / 05 / 16$ \\
\hline$[101]$ & $\gamma=\gamma_{0}$ & NO $\nu \mathrm{A}$ & 3 & {$\left[H_{\text {matter }}^{\text {matter }}, D_{m}\right]=0$} & $03 / 05 / 17$ \\
\hline$[110]$ & $\gamma=\gamma_{0}$ & MINOS+OPERA & 3 & {$\left[H_{\text {matter }}^{\text {matter }}, D_{m}\right]=0$} & $06 / 11 / 17$ \\
\hline$[85]$ & $\gamma=\gamma_{0}$ & DUNE & 3 & No & $19 / 05 / 20$ \\
\hline$[102]$ & $\gamma=\gamma_{0}\left(\frac{E}{G e V}\right)^{n}$ & IceCube & 3 & {$\left[H_{\text {matter }}^{\text {flavor }}, D_{m}\right]=0$} & $21 / 07 / 18$ \\
\hline$[105]$ & $\gamma=\gamma_{0}\left(\frac{E}{G e V}\right)^{n}$ & Super Kamiokande & 2 & {$\left[H_{\text {vacuum }}^{\text {mass }}, D_{m}\right]=0$} & $09 / 06 / 00$ \\
\hline$[104]$ & $\gamma=\gamma_{0} E^{n}$ & Many & 2 & No & $05 / 06 / 01$ \\
\hline$[111]$ & Multiple cases & Many & 3 & No & $16 / 08 / 02$ \\
\hline$[112]$ & Lorentz Invariant & K2K/Super-Kamiokande & 2 & {$\left[H_{\text {vacuum }}^{\text {mass }}, D_{m}\right]=0$} & $07 / 03 / 03$ \\
\hline$[113]$ & $\gamma=\kappa_{n}\left(\frac{E_{\nu}}{G e V}\right)^{n}$ & IceCube & 3 & {$\left[H_{\text {masum }}^{\text {vacuum }}, D_{m}\right]=0$} & $17 / 06 / 05$ \\
\hline
\end{tabular}

Table 4.2: Previous works on neutrino oscillations considering Open System effects.

They showed that a very restricted set of possible Dissipators can be at the same time physical and defined directly in matter. The use of the Dissipator defined directly in the matter basis can result in nonexistent effects at the probability level, as an example, a fake peak was found in reference [109], which led to the belief that the oscillation probabilities were enhanced by the Open System effects.

From table 4.2 we can see that most of the works assumed energy conservation imposing the commutation relation in matter basis ${ }^{5}$, which can be problematic. In particular, the previous work considering the IceCube experiment, reference [102], employed a three-layer formalism under the assumption of energy conservation in the flavor basis to write the $D_{m}$ matrix as a diagonal matrix in the matter basis

$$
D_{m}=\left(\begin{array}{ccc}
d_{m}^{1} & 0 & 0 \\
0 & d_{m}^{2} & 0 \\
0 & 0 & d_{m}^{3}
\end{array}\right),
$$

such that the time evolution of the density operator in matter basis is trivial

\footnotetext{
${ }^{5}$ In the work [106], the authors studied two different cases and assumed energy conservation in only one of them. Since they restricted the study to only two generations the energy conservation condition is valid as we have shown in the chapter [3].
} 


$$
\rho_{i j}(t)=\rho_{i j}(0) e^{-\left(\gamma_{i j}+i \Delta h_{i j}\right) t}
$$

where $\gamma_{i j}=\sum_{m}\left(d_{m}^{i}-d_{m}^{j}\right)^{2}$ and $\Delta h_{i j}=h_{i}-h_{j}$ is the difference between the eigenvalues of the matter basis Hamiltonian. Forgetting the energy conservation issue for a moment, let us investigate if it is possible to reproduce the cases studied in this reference from a valid Kossakowski matrix. In order to do that we have to find the vectorized form of (4.10) with $D_{m}$ written as in (4.12) to compare both approaches. It is given by

$$
\mathcal{D}=\left(\begin{array}{ccccccccc}
0 & 0 & 0 & 0 & 0 & 0 & 0 & 0 & 0 \\
0 & -\gamma_{12} & 0 & 0 & 0 & 0 & 0 & 0 & 0 \\
0 & 0 & -\gamma_{12} & 0 & 0 & 0 & 0 & 0 & 0 \\
0 & 0 & 0 & 0 & 0 & 0 & 0 & 0 & 0 \\
0 & 0 & 0 & 0 & -\gamma_{13} & 0 & 0 & 0 & 0 \\
0 & 0 & 0 & 0 & 0 & -\gamma_{13} & 0 & 0 & 0 \\
0 & 0 & 0 & 0 & 0 & 0 & -\gamma_{23} & 0 & 0 \\
0 & 0 & 0 & 0 & 0 & 0 & 0 & -\gamma_{23} & 0 \\
0 & 0 & 0 & 0 & 0 & 0 & 0 & 0 & 0
\end{array}\right) .
$$

From the Checker map relating the Kossakowski matrix and the Dissipator in three generations (see appendix C), we can infer that the necessary terms on the Kossakowski matrix are $a_{33}, a_{88}$ and $a_{38}$. Imposing that both Dissipators are equal we have the relations

$$
\begin{aligned}
\gamma_{12} & =2 a_{33}, \\
\gamma_{13} & =\frac{1}{2}\left(a_{33}+2 \sqrt{3} a_{38}+3 a_{88}\right), \\
\gamma_{23} & =\frac{1}{2}\left(a_{33}-2 \sqrt{3} a_{38}+3 a_{88}\right) .
\end{aligned}
$$

Three different scenarios were considered in [102]. The first consists of imposing that $\gamma_{13}=$ $\gamma_{23}$ and $\gamma_{12}=0$, this can be recovered in our framework if

$$
\begin{aligned}
& a_{33}=a_{38}=0, \\
& a_{88}=\frac{2}{3} \gamma_{13},
\end{aligned}
$$

where $a_{33}=0$ is a direct consequence of $\gamma_{12}=0$ and $a_{38}$ must be zero to ensure the positivity of the Kossakowski matrix. Therefore, this Dissipator can be reproduced in our description. The 
next case $\gamma_{23}=\gamma_{12}$ and $\gamma_{13}=0$, can be recovered if

$$
a_{38}=-\frac{3 a_{88}+a_{33}}{2 \sqrt{3}}
$$

It is straightforward to check that, for this value, the Cauchy-Schwarz inequality $a_{33} a_{88}-$ $a_{38}^{2} \geq 0$ is violated, which implies that this case is incompatible with the bottom-up approach starting from the Kossakowski matrix. The last case $\gamma_{12}=\gamma_{13}$ and $\gamma_{23}=0$ has the same problem. Therefore, even without considering the problem with energy conservation, we can infer that not all the cases are compatible with the underlying formalism of Open Quantum systems. Moreover, one may question the previous bounds derived in [100, 105, 106]. From the table 4.2 we can see that [100] also assumed energy conservation in matter basis in three generations and the other two studies considered only a simplified two generation description, so it is not clear which parameters they constrained and also how reliable those bounds are.

We can see the differences between the definition of the Dissipator (4.14) in the mass and matter basis by, for example, comparing the corresponding behavior of the oscillation probabilities for a fixed zenith angle. In figures 4.10 (a) and 4.10(b) the probability for $\theta_{z}=\pi$ is shown, it is clear from the figures that the mass basis Dissipator affects much more the oscillation pattern when we increase the value of the Open System parameters in comparison to the Dissipator defined in the matter basis. Furthermore, from the oscillogram 4.11 we can conclude that the difference is not restricted to the zenith angle $\theta_{z}=\pi$, the action of the mass basis Dissipator is much more destructive for all the angles.

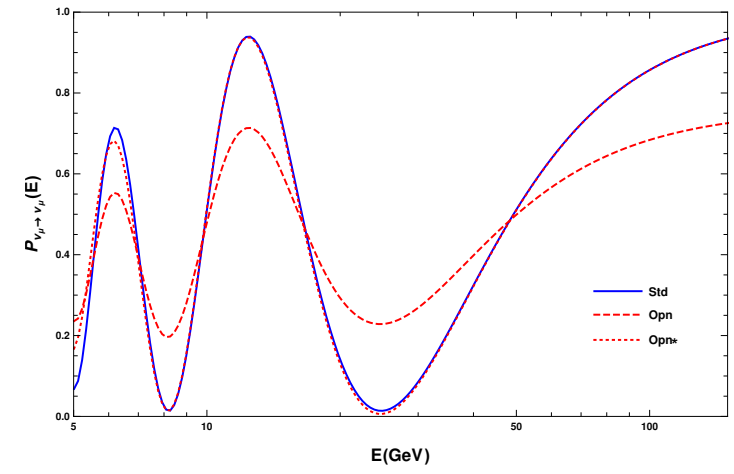

(a) $\nu_{\mu}$ survival probability, for $\theta_{z}=\pi$, comparing the definition of the Dissipator in mass and matter basis (labeled as Opn). The standard oscillation (labeled as Std) is also shown for reference. The $*$ indicates that the probability was derived following reference [102]. The Open System parameters are given by $\gamma_{13}=\gamma_{23}=\frac{2}{3} a_{88}=10^{-23} \mathrm{GeV}$.

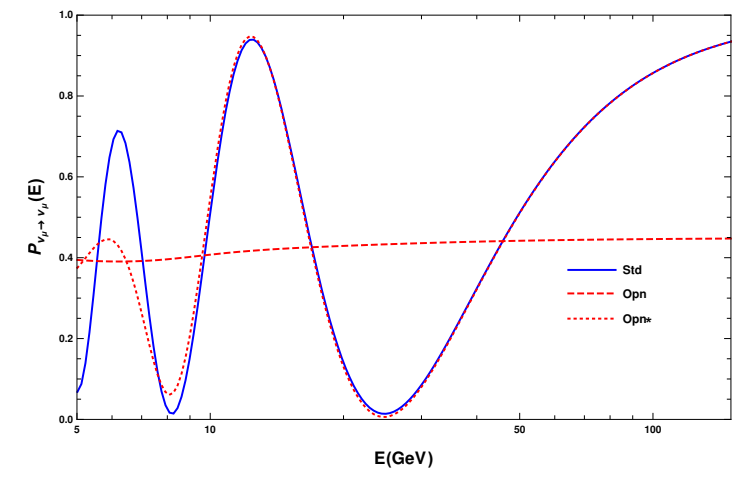

(b) Same as 4.10(a) but for the Open System parameter value $\gamma_{13}=\gamma_{23}=\frac{2}{3} a_{88}=10^{-22} \mathrm{GeV}$.

Figure 4.10: The differences of defining the Dissipator $\mathcal{D}$ in different basis. 
It is important to emphasize that the result was not expected to be similar since we are comparing different situations. The problem with the matter basis Dissipator is that in order for it to be physical, it is necessary to exhibit a mass basis Dissipator which commutes with the Hamiltonian. Then the corresponding matter basis Dissipator $\mathcal{D}$ is obtained by rotating from the mass to the matter basis.

In two-generations, reference [85] showed that it is not possible to find a Dissipator satisfying these restrictions. On the other hand, since in three generations we cannot find analytical expressions the same analysis is very difficult to be performed. We have tried to explicitly write the commutator (4.11) on a mass basis and to impose that each component of the resulting matrix must be zero. This resulted in a trivial $\mathcal{D}_{m}$ which is equivalent to the standard oscillation scenario. This is not a formal analytical derivation, but since in two generations we could not find a physically valid Dissipator satisfying the energy conservation condition, we expect that in three generations it will also be unfeasible.

To conclude, even though the approach starting from the Kossakowski matrix increases the number of free parameters it is easier to guarantee that we have a physical Dissipator. Furthermore, we have the complete positivity conditions 1), 2) and 3) from section 2.2.2 and the Checker map (see appendix C) as a guide to relate the Open System parameters and find the interesting cases. 


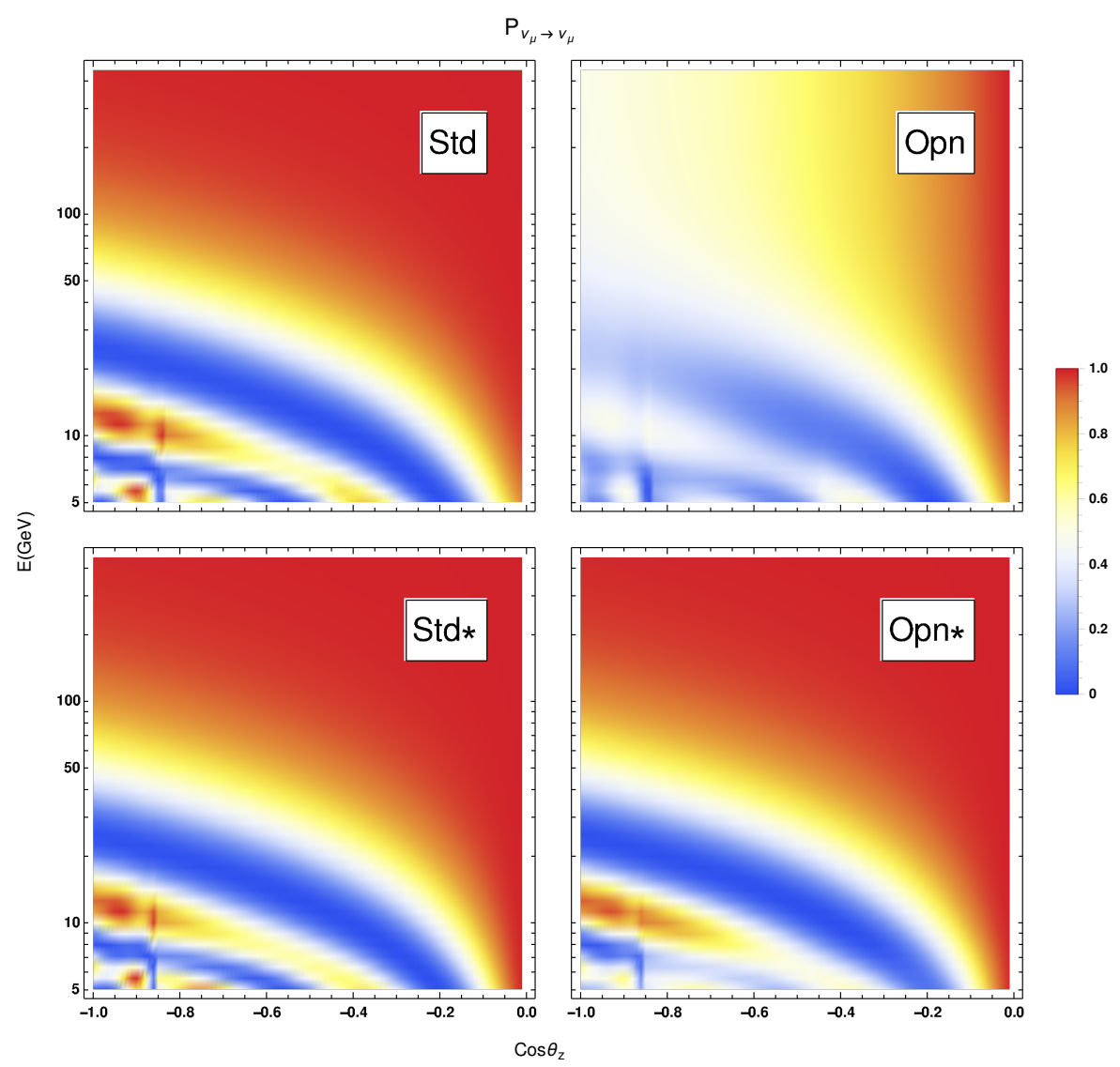

Figure 4.11: $\nu_{\mu}$ survival oscillogram comparing the definition of the Dissipator in mass and matter basis (labeled as Opn). The standard oscillation (labeled as Std) is also shown for reference. The $*$ indicates that the probability was derived following the approach developed in reference [102]. The Open System parameters are fixed to the value $\gamma_{13}=\gamma_{23}=\frac{2}{3} a_{88}=10^{-23} \mathrm{GeV}$. 


\section{CONCLUSIONS AND FUTURE PERSPECTIVES}

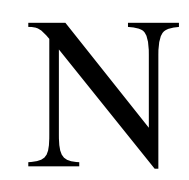

EUTRINOS are one of the building blocks of the Standard Model, the best theory of particles we have so far, even though we are still not able to answer plenty of questions about their properties. The nature of the neutrinos, if they are Dirac or Majorana particles, the absolute neutrino mass scale, the neutrino mass ordering, and the origin of neutrino masses, are among the problems still unanswered.

We have overcome several theoretical and experimental difficulties in the quest for a complete description of the neutrinos. One of the most important discoveries during the development of neutrino physics up to now is the confirmation of the oscillation phenomenon. A neutrino produced in a definite flavor state can change its identity while traveling. This is only possible if these particles are massive, which confirms that the Standard Model must be extended in order to be the final theory of particles as this model does not explain how neutrinos become massive.

Since the oscillation phenomenon is perhaps the best tool we have to study neutrinos, in the first chapter of this dissertation we reviewed in detail the theoretical basis of neutrino oscillations. We discussed three theoretical frameworks for neutrino oscillation in vacuum, the plane wave treatment, the intermediate wave packet description, and the external wave packet description. The most common approach discussed in the literature, the plane wave description, was shown to be physically inconsistent. We have shown that this description can be improved by considering neutrinos as wave packets. The consequence was the presence of a coherence and a localization term. The first determines the extension of the oscillation phenomenon and the second enforces that if we had enough precision to distinguish between neutrino mass eigenstates, we would totally erase the oscillation pattern. Finally, to address the problem that neutrinos 
are never directly measured and to overcome the remaining difficulties regarding the previous description, we discussed the external wave packet framework. It consists of a Quantum Field theoretical approach where the neutrinos are the intermediate states and all amplitudes are written in terms of the external fermions which are described by wave packets. We found that, for neutrinos, we can indeed define an oscillation probability. The final result in this framework is equal to the one obtained in the simpler plane wave approach, which does not imply that the plane wave framework is correct, but only reinforces the fact that neutrinos have special properties.

After having discussed the theoretical basis for vacuum oscillations, we have shown how to include matter effects in the neutrino oscillations. This is crucial since we want to tackle realistic situations where neutrinos travel through the Earth.

In this chapter, we hope to have made it clear how neutrino oscillations is a very sensitive quantum phenomenon.

Motivated by these features of the neutrinos and the confirmation that we have physics beyond the Standard Model, in the second chapter we explored the possibility that the neutrino oscillation pattern can be modified by unknown interactions with the Environment if we treat neutrinos as an Open Quantum System. This is an extension of the Quantum Mechanical treatment for a closed system in order to study coupled systems. For this purpose, we introduced the density operator, a very useful tool for the study of Open Systems. We described the relevant properties of the density operator, and studied how the subsystem dynamics, in our case the neutrinos, would change due to its internal dynamics plus the correlations acquired through the interactions with the Environment. We have shown that the change consists of including an extra term in the Von Neumann equation, the Dissipator $\mathcal{D}$. This is a theoretically appealing framework because the equation dictating the reduced dynamics is applicable regardless of what might be the form of the interaction between the neutrino and the Environment. We have shown that the evolution of the neutrino subsystem can be obtained by a Universal Dynamical Map under some reasonable assumptions. This map is a trace-preserving linear completely positive map so it has a Krauss decomposition. In this case, we saw that our ignorance is encoded in a matrix of Open System parameters, called the Kossakowski matrix. This makes the Open System framework extremely useful for a phenomenological approach.

To conclude the chapter, we discussed three distinct quantum entropies which are auxiliary tools to understand the consequences of the Open System interactions.

Hitherto, we have discussed the Open System effects and the neutrino oscillations separately. In the third chapter, we finally explore the consequences of taking into account both effects together. We started by considering a generic two-dimensional description in order to 
show how to deal with the Open System effects in a practical example. This generic example is then adapted to the study of vacuum neutrino oscillations in two generations to explore the consequences of Open System effects in the simplest possible scenario. Despite the limitations of this description, we were able to find interesting differences in the oscillation pattern. First, the Open System effects introduce damping terms in the probabilities, in such a manner that as we increase the value of the Open System parameters the oscillations are erased.

Also, when Open System effects are considered, the only available Majorana phase in two generations, unimportant in the standard scenario, becomes relevant for the oscillation probabilities. This allows us to distinguish, at least at the probability level, between Dirac and Majorana neutrinos. Furthermore, in one of the cases we have studied, the dependence of the probabilities with the Majorana phase is through an odd function in such a manner that the CP symmetry is violated.

In this simplified framework, we also introduced a new tool that we have developed, the Checker map, which is very useful in order to see what terms of the Dissipator can be turned on, given a Kossakowski matrix.

Finally, we moved to the more realistic description of neutrino oscillations in three generations in matter. We always considered the energy range and the baseline length compatible with the reach of the IceCube experiment, the experiment we want to use in order to study the consequences of Open System effects in atmospheric neutrino oscillations. We were able to find interesting differences in the oscillation pattern in comparison to the standard scenario.

The $\nu_{\mu}$ survival probability was damped by the Open System effects which imply that at least one of the other oscillation channels must be enhanced. We found that the $\nu_{\mu} \rightarrow \nu_{e}$ probability is significantly increased. This is extremely relevant for the IceCube experiment because an excess of $\nu_{e}$ induced events is a signal that IceCube can search for once they may leave different signatures in the detector in comparison to $\nu_{\mu}$ induced events.

Another consequence of the Open System effects is related to the allowed phases in the mixing matrix. For Dirac neutrinos, we have only one phase, which we called $\delta$, while for Majorana neutrinos, in addition to $\delta$, we have two extra phases $\phi_{1}$ and $\phi_{2}$. When the Open System effects are present we saw that all the three phases are relevant and have noticeable consequences at the probability level.

The analysis of $\mathrm{CP}$ violation in this situation is more complicated since, as we have shown, matter effects already violate the CP symmetry even in the standard scenario. The violation solely due to the Open System effects can be studied considering the high energy regime, where the matter effects become irrelevant. But in this energy range, the violation is small.

We also studied the entropy for different Majorana phases. As in two generations, Open 
System effects drive the initial pure state into a mixed one with the effect of the Majorana phases restricted just to change how mixed the final state is.

For reference and to show how IceCube is a better choice in the search for Open System effects we concluded the chapter by showing different Open System scenarios considering the changes in the survival probability for the $\mathrm{NO} \nu \mathrm{A}$ experiment. It is interesting because $\mathrm{NO} \nu \mathrm{A}$ is the laboratory experiment with the longest baseline currently taking data. With this comparison, we were able to see how IceCube is more sensitive to Open System effects.

In possession of all of these results, in chapter four, we finally were able to tackle the challenge of reproducing IceCube atmospheric neutrino data in order to compare with their results, our Open System framework predictions. We started by a detailed discussion of the IceCube experiment and the data sets we have used in our analysis. All of the detector and data related issues were explained.

Then we briefly discussed atmospheric neutrinos, this was important because we chose to use this source of neutrinos in our study and also to settle a few details concerning our simulation.

After discussing the detector and the neutrino source we moved to the discussion of the model we used to calculate the oscillation probabilities. We introduced a generic $\mathrm{N}$ layer model, which consists of slicing the Earth in layers of constant density and evolving the neutrino state through each layer. In particular, for our simulation we considered $\mathrm{N}=5$. This was the only missing piece to finally compute our predictions.

We then discussed the statistical analysis we have used to test our predictions. The goodness of fit of each model was evaluated by considering a $\chi^{2}$ test statistics. The variation of the $\chi^{2}$ under repeated simulated experiments was used to define the $95 \%$ allowed region of the Open System parameters.

By comparing our predictions, considering the standard oscillation scenario with the neutrino oscillation parameters fixed to the NuFIT best fit values, with the IceCube data we found that our computation is in very good agreement with their measurements.

Finally, we tested the predictions for the Open System framework. The Open System scenario we chose to study was defined through the Kossakoski matrix. Our particular choice was based on the restrictions of the parameters in this matrix from the requirement of complete positivity. The analysis of the sensitivity of the $\chi^{2}$ allowed us to put stringent bounds on the elements of the Kossakowski matrix, we obtained at $95 \%$ confidence level

$$
a_{i i} \leq 1.38 \times 10^{-25} \mathrm{GeV}
$$

We hope to have made it clear how the definitions starting from the Kossakowski matrix 
establish a standard procedure by which future works on this subject can be easily compared even if other scenarios and experiments are considered.

To conclude, we discussed previous works on the Open system effects in neutrino oscillations. Since we have always emphasized the importance of the underlying formalism of Open Quantum Systems, in this section, we have shown inconsistencies of previous works in implementing Open System effects.

Since we have entered a precision measurement era for neutrino oscillation parameters, the future of neutrino physics is very promising. We hope to discover very interesting features of these particles in the next years. A planned upgrade of the IceCube detector is about to happen and new experiments such as DUNE have ambitious proposals to contribute to the challenging quest to understanding neutrinos.

Open System effects can then be further constrained or even confirmed in the following years. Furthermore, we have thought in different manners of searching for these effects during the development of this work.

The IceCube experiment is able to reconstruct at the statistical level the number of $\nu_{\tau}$ events. As we have shown in this dissertation, the Open System effects can decrease the number of such events, so in principle, the collaboration could search for this discrepancy. Also, an excess of $\nu_{e}$ events might also be a signal of Open System effects as we have already stated.

Besides that, since the entropy of the state change solely due to the Open System interactions we want to explore the possibility of relating this quantity to an observable. If we succeed, it can be another useful tool in the search for the Open System effects.

Also, we are planning to extend our work in order to consider neutrinos coming from astrophysical sources. It is not clear by now if the Open System effects have interesting consequences in this scenario.

Without a doubt, neutrinos are one of the best choices we have at the moment to make further progress in particle physics. With the constant improvement of the experimental setup, we are only bounded by our creativity to unravel their secrets. For sure, neutrinos will probably reveal to us features of the universe that we could not even dream of. The possibility of helping in this saga is incredibly exciting. 


\section{APPENDIX A}

\section{NEUTRINO OSCILLATIONS IN DIFFERENT PRESCRIPTIONS}

In this appendix we will discuss in more detail the calculations of chapter [1].

\section{A.1 Oscillations in the internal wave packet description}

Here we will derive the probability for neutrino oscillations in the intermediate wave packet model.

Writing (1.11) explicitly

$$
\begin{aligned}
\mathcal{P}_{\nu_{\alpha} \rightarrow \nu_{\beta}} & =\operatorname{Tr}\left\{\sum_{i, j} U_{\beta i}^{*} U_{\beta j} \int[d p] \int\left[d p^{\prime}\right] f_{\nu_{j}}(\overrightarrow{\mathrm{p}}, \overrightarrow{\mathrm{P}}) f_{\nu_{j}}^{*}(\overrightarrow{\mathrm{p}}, \overrightarrow{\mathrm{P}}) e^{-i\left(E_{i}-E_{j}\right)\left(t-t_{p}\right)+i\left(\overrightarrow{\mathrm{p}}-\overrightarrow{\mathrm{p}}^{\prime}\right) \cdot \overrightarrow{\mathrm{x}}_{p}}\left|\nu_{i}, \overrightarrow{\mathrm{p}}\right\rangle\left\langle\nu_{j}, \overrightarrow{\mathrm{p}}^{\prime}\right|\right. \\
& \left.\sum_{k, l} U_{\beta k}^{*} U_{\beta l} \int[d k] \int\left[d k^{\prime}\right] f_{\nu_{k}}(\overrightarrow{\mathrm{k}}, \overrightarrow{\mathrm{P}}) f_{\nu_{l}}^{*}\left(\overrightarrow{\mathrm{k}}^{\prime}, \overrightarrow{\mathrm{P}}\right) e^{i\left(\overrightarrow{\mathrm{k}}-\overrightarrow{\mathrm{k}}^{\prime}\right) \cdot \overrightarrow{\mathrm{x}}_{p}}\left|\nu_{k}, \vec{k}\right\rangle\left\langle\nu_{l}, \vec{k}^{\prime}\right|\right\} .
\end{aligned}
$$

Using the normalization condition (1.7) we are left with one sum and two integrals

$$
\begin{aligned}
\mathcal{P}_{\nu_{\alpha} \rightarrow \nu_{\beta}} & =\sum_{i, j} U_{\beta i}^{*} U_{\beta j} U_{\alpha j}^{*} U_{\alpha i} \int[d p] \int\left[d p^{\prime}\right] f_{\nu_{i}}(\overrightarrow{\mathrm{p}}, \overrightarrow{\mathrm{P}}) f_{\nu_{j}}^{*}\left(\overrightarrow{\mathrm{p}}^{\prime}, \overrightarrow{\mathrm{P}}\right) f_{\nu_{j}}\left(\overrightarrow{\mathrm{p}}^{\prime}, \overrightarrow{\mathrm{P}}\right) f_{\nu_{i}}^{*}(\overrightarrow{\mathrm{p}}, \overrightarrow{\mathrm{P}}) \\
& \times e^{-i\left(E_{i}-E_{j}\right) T+i\left(\overrightarrow{\mathrm{p}}-\overrightarrow{\mathrm{p}}^{\prime}\right) \cdot \overrightarrow{\mathrm{x}}_{D}-i\left(\overrightarrow{\mathrm{p}}-\overrightarrow{\mathrm{p}}^{\prime}\right) \cdot \overrightarrow{\mathrm{x}}_{p}}
\end{aligned}
$$


where the detected neutrino is described by a wave packet peaked at the detection position $\vec{x}_{D}$ and $T=t_{D}-t_{P}$ is the time interval between production and detection. To perform the integral we will assume that the distribution function $f_{\nu_{j}}(\overrightarrow{\mathrm{p}}, \overrightarrow{\mathrm{P}})$ is sharply peaked at the mean momentum $\overrightarrow{\mathrm{P}}$ and approximate it by a Gaussian wave packet with momentum width $\sigma_{p, i}$, where the label $i$ is necessary to distinguish between production $P$ and detection $D$

$$
f_{\nu_{j}}(\overrightarrow{\mathrm{p}}, \overrightarrow{\mathrm{P}})=\left(\frac{2 \pi}{\sigma_{p, i}^{2}}\right)^{3 / 4} \exp \left(\frac{-(\overrightarrow{\mathrm{p}}-\overrightarrow{\mathrm{P}})^{2}}{4 \sigma_{p, i}^{2}}\right) .
$$

The convolution of the gaussian distribution functions establishes the effective momentum uncertainty:

$$
\begin{aligned}
f_{\nu_{j}}(\overrightarrow{\mathrm{p}}, \overrightarrow{\mathrm{P}}) f_{\nu_{j}}^{*}(\overrightarrow{\mathrm{p}}, \overrightarrow{\mathrm{P}}) & =\left(\frac{2 \pi}{\sigma_{p, P}^{2}}\right)^{3 / 4}\left(\frac{2 \pi}{\sigma_{p, D}^{2}}\right)^{3 / 4} \exp \left(\frac{-(\overrightarrow{\mathrm{p}}-\overrightarrow{\mathrm{P}})^{2}}{4}\left(\frac{1}{\sigma_{p, P}^{2}}+\frac{1}{\sigma_{p, D}^{2}}\right)\right), \\
& =\left(\frac{2 \pi}{\sigma_{p, P}^{2}}\right)^{3 / 4}\left(\frac{2 \pi}{\sigma_{p, D}^{2}}\right)^{3 / 4} \exp \left(\frac{-(\overrightarrow{\mathrm{p}}-\overrightarrow{\mathrm{P}})^{2}}{4 \sigma_{p}^{2}}\right) .
\end{aligned}
$$

Where

$$
\sigma_{p}^{2}=\left(\frac{1}{\sigma_{p, P}^{2}}+\frac{1}{\sigma_{p, D}^{2}}\right)
$$

Inserting the result back into (A.1)

$$
\begin{aligned}
\mathcal{P}_{\nu_{\alpha} \rightarrow \nu_{\beta}} & =\sum_{i, j} U_{\beta i}^{*} U_{\beta j} U_{\alpha j}^{*} U_{\alpha i} \int \frac{d \overrightarrow{\mathrm{p}}^{3}}{(2 \pi)^{3}} \int \frac{d \overrightarrow{\mathrm{p}}^{3}}{(2 \pi)^{3}}\left(\frac{2 \pi}{\sigma_{p, p}^{2}}\right)^{3 / 2}\left(\frac{2 \pi}{\sigma_{p, D}^{2}}\right)^{3 / 2} \\
& \times \exp \left(\frac{-(\overrightarrow{\mathrm{p}}-\overrightarrow{\mathrm{P}})^{2}}{4 \sigma_{p}^{2}}\right) \exp \left(\frac{-\left(\overrightarrow{\mathrm{p}}^{\prime}-\overrightarrow{\mathrm{P}}\right)^{2}}{4 \sigma_{p}^{2}}\right) e^{-i\left(E_{j}-E_{i}\right) T+i\left(\overrightarrow{\mathrm{p}}-\overrightarrow{\mathrm{p}}^{\prime}\right) \cdot\left(\overrightarrow{\mathrm{x}}_{D}-\overrightarrow{\mathrm{x}}_{p}\right)}
\end{aligned}
$$

Once the wave packet is sharply peaked at the mean momentum $\overrightarrow{\mathrm{P}}$ we can expand the energy around the mean momenta

$$
E_{i}(p) \approx E(P)+\overrightarrow{\mathrm{v}}_{i} \cdot(\overrightarrow{\mathrm{p}}-\overrightarrow{\mathrm{P}})
$$

where $\overrightarrow{\mathrm{v}}_{i}=\frac{\overrightarrow{\mathrm{P}}}{E_{i}(\mathrm{P})}$ is the group velocity. Now we are in position to compute the Gaussian integrals with the help of the following identity 


$$
\int \exp \left(-\frac{1}{2} \overrightarrow{\mathrm{x}}^{T} \cdot A \cdot \overrightarrow{\mathrm{x}}+J \cdot \overrightarrow{\mathrm{x}}\right) d x^{n}=\sqrt{\frac{(2 \pi)^{n}}{\operatorname{det}(A)}} \exp \left(\frac{1}{2} J^{T} \cdot A^{-1} \cdot J\right) .
$$

Collecting the terms with $\vec{p}$ and using the above relation

$$
\begin{gathered}
\int d \overrightarrow{\mathrm{p}} \exp \left(-\frac{\left(\overrightarrow{\mathrm{p}}-\overrightarrow{\mathrm{p}}^{\prime}\right)^{2}}{4 \sigma_{p}^{2}}\right) \exp \left(-i \overrightarrow{\mathrm{v}}_{j} \cdot(\overrightarrow{\mathrm{p}}-\overrightarrow{\mathrm{P}}) T+i \overrightarrow{\mathrm{p}} \cdot \overrightarrow{\mathrm{L}}\right) \\
=\left(8\left(2 \pi \sigma_{p}^{2}\right)^{3}\right)^{1 / 2} \exp \left(-\frac{\left(\overrightarrow{\mathrm{L}}-\overrightarrow{\mathrm{v}}_{j} T\right)^{2}}{4 \sigma_{x}^{2}}+i \overrightarrow{\mathrm{P}} \cdot \overrightarrow{\mathrm{L}}\right)
\end{gathered}
$$

where $\overrightarrow{\mathrm{L}}=\overrightarrow{\mathrm{x}}_{D}-\overrightarrow{\mathrm{x}}_{P}$ and we used the Heisenberg uncertainty relation $\sigma_{p} \sigma_{x}=1 / 2$. The integral over $\overrightarrow{\mathrm{p}}^{\prime}$ can be performed by the same procedure

$$
\begin{gathered}
\int d \overrightarrow{\mathrm{p}} \exp \left(-\frac{\left(\overrightarrow{\mathrm{p}}-\overrightarrow{\mathrm{p}}^{\prime}\right)^{2}}{4 \sigma_{p}^{2}}\right) \exp \left(i \overrightarrow{\mathrm{v}}_{j} \cdot(\overrightarrow{\mathrm{p}}-\overrightarrow{\mathrm{P}}) T-i \overrightarrow{\mathrm{p}} \cdot \overrightarrow{\mathrm{L}}\right) \\
=\left(8\left(2 \pi \sigma_{p}^{2}\right)^{3}\right)^{1 / 2} \exp \left(-\frac{\left(\overrightarrow{\mathrm{L}}-\overrightarrow{\mathrm{v}}_{j} T\right)^{2}}{4 \sigma_{x}^{2}}-i \overrightarrow{\mathrm{P}} \cdot \overrightarrow{\mathrm{L}}\right)
\end{gathered}
$$

Thus we have so far

$$
\begin{aligned}
\mathcal{P}_{\nu_{\alpha} \rightarrow \nu_{\beta}} & =\sum_{i, j} U_{\beta i}^{*} U_{\beta j} U_{\alpha j}^{*} U_{\alpha i} \frac{8\left(\sigma_{p}^{2}\right)^{3}}{\left(\sigma_{p, P}^{2} \sigma_{p, D}^{2}\right)^{3 / 2}} \\
& \times \exp \left(-\frac{\left(\overrightarrow{\mathrm{L}}-\overrightarrow{\mathrm{v}}_{i} T\right)^{2}}{4 \sigma_{x}^{2}}-\frac{\left(\overrightarrow{\mathrm{L}}-\overrightarrow{\mathrm{v}}_{j} T\right)^{2}}{4 \sigma_{x}^{2}}\right) .
\end{aligned}
$$

Most experiments do not measure the production and detection times, the standard procedure is to average over $T$, which gives again a Gaussian integral

$$
\int \mathcal{P}_{\nu_{\alpha} \rightarrow \nu_{\beta}} d T=\left(\frac{(2 \pi)\left(2 \sigma_{x}^{2}\right)}{v_{i}^{2}+v_{j}^{2}}\right) \exp \left[\left(\frac{\left(v_{i}+v_{j}\right) L}{2 \sigma_{x}^{2}}-i \Delta E_{i j}\right)^{2}\left(\frac{\sigma_{x}^{2}}{\left(v_{i}^{2}+v_{j}^{2}\right)}\right)-\frac{L^{2}}{2 \sigma_{x}^{2}}\right]
$$

where we used the fact that the Neutrino propagation path is aligned with its velocity and employed the shorthand notation $\left|\overrightarrow{\mathrm{v}}_{i}\right|=v_{i},|\overrightarrow{\mathrm{L}}|=L$. Now assuming that the energy differences are 
smaller compared to their average values, we can expand

$$
\Delta E=\frac{\partial E}{\partial p} \Delta p+\frac{\partial E}{\partial m^{2}} \Delta m^{2}=v \Delta P+\frac{1}{2 E} \Delta m^{2}=\frac{1}{2 E} \Delta m^{2} .
$$

Under this assumption we can also approximate the group velocities by

$$
v_{i}=1-\frac{m_{i}^{2}}{2 E^{2}}
$$

In (A.11) we have three different kind of terms to analyze

$$
\begin{aligned}
\frac{\left(v_{i}+v_{j}\right)^{2}-v_{i}^{2}-v_{j}^{2}}{v_{i}^{2}+v_{j}^{2}} & \approx-\frac{\left(\Delta m_{i j}^{2}\right)^{2}}{8 E^{4}}, \\
\frac{\left(v_{i}+v_{j}\right) \Delta E_{i j}}{v_{i}^{2}+v_{j}^{2}} & \approx \frac{\Delta m_{i j}^{2}}{2 E}, \\
\frac{\left(\Delta E_{i j}\right)^{2}}{v_{i}^{2}+v_{j}^{2}} & \approx \frac{\left(\Delta E_{i j}\right)^{2}}{2} .
\end{aligned}
$$

So plugging the above into (A.11) we have

$$
\begin{aligned}
\mathcal{P}_{\nu_{\alpha} \rightarrow \nu_{\beta}} & \propto \sum_{i, j} U_{\beta i}^{*} U_{\beta j} U_{\alpha j}^{*} U_{\alpha i} \exp \left(-\left(\frac{\Delta m_{i j}^{2}}{4 \sqrt{2} \sigma_{x} E} L\right)^{2}-i \frac{\Delta m_{i j}^{2}}{2 E} L-\left(\frac{\Delta m_{i j}^{2}}{2 \sqrt{2} E} \sigma_{x}\right)^{2}\right) \\
& \propto \sum_{i, j} U_{\beta i}^{*} U_{\beta j} U_{\alpha j}^{*} U_{\alpha i} \exp \left(-2 \pi i \frac{L}{L_{i j}^{o s c}}-\left(\frac{L}{L_{i j}^{c o h}}\right)^{2}-2 \pi^{2}\left(\frac{\sigma_{x}}{L_{i j}^{o s c}}\right)^{2}\right)
\end{aligned}
$$

Where

$$
\begin{aligned}
L_{i j}^{\mathrm{osc}} & \equiv \frac{4 \pi E}{\Delta m_{i j}^{2}}, \\
L_{i j}^{\mathrm{coh}} & \equiv \frac{4 \sqrt{2} E^{2}}{\left|\Delta m_{i j}^{2}\right|} \sigma_{x} .
\end{aligned}
$$

\section{A.2 Oscillations in the external wave packet description}

Here we will derive the equations (1.28), (1.29), and discuss other results from chapter [1]. The diagrams representing production and detection are:

The differential probability is given by 


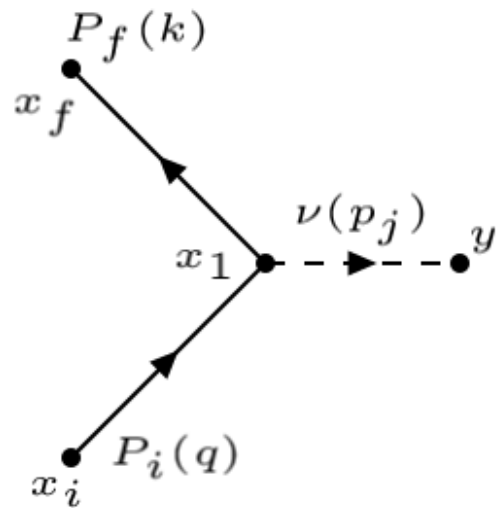

(a) Production process.

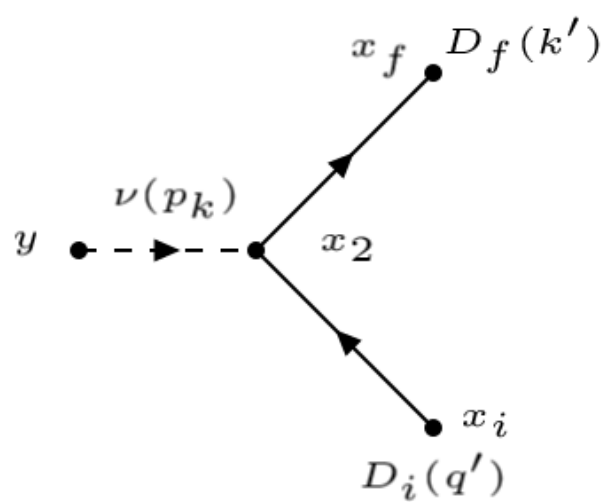

(b) Detection process.

$$
d P=\frac{|\langle f|S| i\rangle|^{2}}{\langle f \mid f\rangle\langle i \mid i\rangle} d \Pi,
$$

where

$$
d \Pi=\frac{V}{(2 \pi)^{3}} d^{3} p_{j},
$$

is the phase space volume. Expanding the amplitude (1.16) to first order

$$
\langle f|S| i\rangle=-i \int d^{4} x\left\langle f\left|H_{I}\right| i\right\rangle
$$

where the final $|f\rangle$ and initial $|i\rangle$ states are given by

$$
\begin{aligned}
|f\rangle & =|\nu\rangle\left|P_{f}\right\rangle, \\
|i\rangle & =\left|P_{i}\right\rangle .
\end{aligned}
$$

Using the wave packet decomposition (1.15) we have that

$$
\langle f|S| i\rangle=-i \int[d k] \int[d q] \int d^{4} x f_{P, i}(q, Q) f_{P, f}(k, K)\left\langle\nu\left(p_{j}\right) P_{f}(k)\left|H_{I}\right| P_{i}(q)\right\rangle
$$

The amplitude $\left\langle\nu\left(p_{j}\right) P_{f}(k)\left|H_{I}\right| P_{i}(q)\right\rangle$ is the plane wave amplitude and can be calculated by the LSZ reduction formula [64] 


$$
\begin{array}{r}
\langle f|S| i\rangle=\left[i \int d^{4} x_{i} e^{-i q x_{i}}\left(\square^{2}+m_{i}^{2}\right)\right]\left[i \int d^{4} x_{f} e^{i k x_{f}}\left(\square^{2}+m_{f}^{2}\right)\right] \\
{\left[i \int d^{4} y e^{-i p_{j} y}\left(\square^{2}+m_{j}^{2}\right)\right] U_{\alpha j}^{*} M_{P, j}(q, k) D_{x_{i} x_{1}} D_{1 x_{f}} D_{x_{1} y} .}
\end{array}
$$

Where $M_{P, j}{ }^{1}$ is the matrix element associated with the vertex and each $D$ is a propagator represented by

$$
D_{x y}=\int \frac{d^{4} p}{(2 \pi)^{4}} \frac{i}{p^{2}-m^{2}+i \epsilon} e^{i p(x-y)} .
$$

When multiplied by (A.23) the poles cancel, then

$$
\begin{aligned}
\langle f|S| i\rangle & =-i \int[d k] \int[d q] \int d^{4} x f_{P, i}(q, Q) f_{P, f}(k, K) \int x_{i} e^{-i q x_{i}} \int d^{4} x_{f} e^{i k x_{f}} \\
& \times \int d^{4} y e^{i p_{j} y} U_{\alpha j}^{*} M_{D, j}(q, k) \int \frac{d^{4} p}{(2 \pi)^{4}} e^{i p\left(x_{i}-x\right)} \int \frac{d^{4} p^{\prime}}{(2 \pi)^{4}} e^{i p^{\prime}\left(x-x_{f}\right)} \\
& \times \frac{d^{4} k^{\prime}}{(2 \pi)^{4}} e^{i k^{\prime}(x-y)} .
\end{aligned}
$$

The integrals over $x_{i}, x_{f}$ an $y$ result in delta functions

$$
\begin{aligned}
\langle f|S| i\rangle=-i & \int d^{4} x \int[d q] \int[d k] f_{P, i}(q, Q) f_{P, f}(k, K) \int \frac{d^{4} p}{(2 \pi)^{4}} \frac{d^{4} p^{\prime}}{(2 \pi)^{4}} \frac{d^{4} k^{\prime}}{(2 \pi)^{4}} U_{\alpha j}^{*} M_{j}(q, k) \\
& \times(2 \pi)^{4} \delta^{4}(q-p)(2 \pi)^{4} \delta^{4}\left(k-p^{\prime}\right)(2 \pi)^{4} \delta^{4}\left(p_{j}-k^{\prime}\right) e^{-i\left(p-p^{\prime}-k^{\prime}\right) x} .
\end{aligned}
$$

The integration over $p, p^{\prime}$ and $k^{\prime}$ can be then easily evaluated

$$
\langle f|S| i\rangle=-i U_{\alpha j}^{*} \int d^{4} x \int[d q] \int[d k] f_{P, i}(q, Q) f_{P, j}(k, K) M_{D, j}(q, k) e^{-i\left(q-k-p_{j}\right) x} .
$$

The result above, apart from the $U_{\alpha j}^{*}$ factor, is exactly the definition of $\phi_{P, j}\left(E, \overrightarrow{\mathrm{p}}_{j}\right)$ in (1.22). The squared amplitude for the production process is then obtained by squaring the above result and summing over all possible states $j$

\footnotetext{
${ }^{1}$ Here the factor $\sqrt{2 p_{0}}$ is not included yet.
} 


$$
|\langle f|S| i\rangle|^{2}=\sum_{j}\left|U_{\alpha j}\right|^{2}\left|\phi_{P, j}\left(E, \overrightarrow{\mathrm{p}}_{j}\right)\right|^{2}
$$

Now we must calculate the normalization of the initial and final states. Any state composed of a wave packet is already normalized due to equation (1.7), so only the plane wave outgoing neutrino has a nontrivial contribution ${ }^{2}$, which is given by [64]

$$
\langle\nu \mid \nu\rangle=2 E V
$$

where $E$ is the neutrino energy and $V$ is the normalization volume. Putting everything together the differential probability is

$$
d P=\sum_{j}\left|U_{\alpha j}\right|^{2} \frac{\left|\phi_{D, j}\left(E, \overrightarrow{\mathrm{p}}_{j}\right)\right|^{2}}{2 E V} \frac{V}{(2 \pi)^{3}} d^{3} p_{j} .
$$

The factor $1 / 2 E$ can be absorbed into the definition of $M_{D, j}$ (see (1.19)). Assuming that the production is spherically symmetric and changing variables from $p_{j}$ to $E$ we have the desired result (1.28)

$$
P_{\alpha}^{\text {prod }}=\sum_{j}\left|U_{\alpha j}\right|^{2} \frac{1}{8 \pi^{2}} \int d E\left|\phi_{P, j}\left(E . p_{j}\right)\right|^{2} 4 E p_{j} .
$$

For the production process the steps are the same the only difference is that the neutrino is in the initial state (see figure (A.1(b))), the factor $\langle f|S| i\rangle$ is then independent of the phase space volume. Since $\int d \Pi=1$ the result is

$$
P_{\beta}^{\text {det }}=\sum_{k}\left|U_{\beta k}\right|^{2}\left|\phi_{D, k}\left(E, p_{k}\right)\right|^{2} \frac{1}{V}
$$

\section{A.2.1 The Grimus-Stockinger theorem:}

The Grimus-Stockinger theorem states that, in the limit $L \gg p_{j} / \sigma_{p}^{2}$ the following result is valid

$$
\int d^{3} \overrightarrow{\mathrm{p}} \frac{\psi(\overrightarrow{\mathrm{p}}) e^{i \overrightarrow{\mathrm{p}} \overrightarrow{\mathrm{L}}}}{A-\overrightarrow{\mathrm{p}}^{2}+i \epsilon}=-\frac{2 \pi^{2}}{L} \psi\left(\sqrt{A} \frac{\overrightarrow{\mathrm{L}}}{L}\right) e^{i \sqrt{A} L} .
$$

This result was used to calculate the amplitude for the oscillation probability (1.26).

\footnotetext{
${ }^{2}$ Note that in this framework the internal state is a plane wave while the external states are wave packets.
} 


\section{APPENDIX B}

\section{ANALYTICAL RESULTS FOR OPEN SYSTEMS}

In this appendix we will derive equation (3.6) and prove the fact that if the first column of $\mathcal{D}$ is zero the von Neumann entropy increases in time.

\section{B.1 A sufficient condition for the entropy to increase}

To prove the desired result we will use the following condition [114]:

For a complete positive trace preserving map $V$ the relative entropy satisfies, for any two states $\rho, \sigma$

$$
S(V(\rho) \| V(\sigma)) \leq S(\rho \| \sigma) .
$$

Choosing $\sigma$ as the maximally mixed state $\frac{1}{N} \mathcal{I}_{N \times N}$, we have that if it is a fixed point of the evolution

$$
\mathcal{D}\left[\frac{1}{N} \mathcal{I}_{N \times N}\right]=0
$$

We are done, because

$$
S(V(\rho) \| V(\sigma))=S(V(\rho) \| \sigma) \leq S(\rho \| \sigma)
$$

which implies 


$$
\begin{aligned}
-S(V(\rho))-\operatorname{Tr}\{V(\rho) \ln \sigma\} & \leq-S(\rho)-\operatorname{Tr}\{\rho \ln \sigma\}, \\
-S(V(\rho))-\operatorname{Tr}\left\{V(\rho) \ln \frac{1}{N} \mathcal{I}_{N \times N}\right\} & \leq-S(\rho)-\operatorname{Tr}\left\{\rho \ln \frac{1}{N} \mathcal{I}_{N \times N}\right\}, \\
-(S(V(\rho))-S(\rho)) & \leq-\operatorname{Tr}\{V(\rho) \ln N\}+\operatorname{Tr}\{\rho \ln N\}, \\
\Delta S(\rho) & \geq 0,
\end{aligned}
$$

where we used the fact that $V$ is a trace-preserving map. The maximally mixed state can be decomposed as $\sigma=\frac{1}{N} \mathcal{I}_{N \times N}$, so in vectorized form the condition (B.2) can be written as

$$
V(\hat{\sigma})=\left[\begin{array}{ccc}
0 & \ldots & 0 \\
V_{11} & \cdots & V_{1 N-1} \\
\vdots & \ddots & \vdots \\
V_{1 N-1} & \ldots & V_{N^{2}-1 N^{2}-1}
\end{array}\right]\left(\begin{array}{c}
1 \\
0 \\
\vdots \\
0
\end{array}\right)
$$

Hence, if the first column is zero, the condition (B.2) is satisfied and the entropy increases with time! For a different derivation see [82].

\section{B.2 The two-dimensional Lindbladian}

To write $\mathcal{D}$ as in equation (3.5) we have to write the density operator in component form $\rho=\rho_{\mu} \sigma_{\mu}, \mu=0,1,2,3$, so that

$$
\begin{aligned}
\mathcal{D}[\rho(t)] & =\frac{1}{2} \sum_{i, j=1}^{3} a_{i j}\left[\sum_{\mu}\left(2 \sigma_{i} \sigma_{\mu} \sigma_{j} \rho_{\mu}-\sigma_{j} \sigma_{i} \sigma_{\mu} \rho_{\mu}-\rho_{\mu} \sigma_{\mu} \sigma_{j} \sigma_{i}\right)\right], \\
& =\frac{1}{2} \sum_{i, j=1}^{3}[2 \rho_{0} \underbrace{\left(\sigma_{i} \sigma_{j}-\sigma_{j} \sigma_{i}\right)}_{\mu=0}+\sum_{t=1}^{3}\left(2 \sigma_{i} \sigma_{j} \sigma_{t} \rho_{t}-\sigma_{j} \sigma_{i} \sigma_{t} \rho_{t}-\rho_{t} \sigma_{t} \sigma_{j} \sigma_{i}\right)] .
\end{aligned}
$$

The terms with summation over $t$ can be reduced by using multiple times the anticommutation relations of the Pauli matrices $\sigma_{i} \sigma_{j}=2 \delta_{i j}-\sigma_{j} \sigma_{i}$ 


$$
\begin{aligned}
& 2 \sigma_{i} \sigma_{t} \sigma_{j}-\sigma_{j} \sigma_{i} \sigma_{t}-\sigma_{t} \sigma_{j} \sigma_{i} \\
& =2 \sigma_{i} \sigma_{t} \sigma_{j}-\left(2 \delta_{j i}-\sigma_{i} \sigma_{j}\right) \sigma_{t}-\sigma_{t}\left(2 \delta_{j i}-\sigma_{i} \sigma_{j}\right) \\
& =2 \sigma_{i} \sigma_{t} \sigma_{j}-2 \delta_{j i} \sigma_{t}+\sigma_{i}\left(2 \delta_{j t}-\sigma_{t} \sigma_{j}\right)-2 \delta_{j i} \sigma_{t}+\left(\delta_{i t}-\sigma_{i} \sigma_{t}\right) \sigma_{j}, \\
& =2 \sigma_{i} \sigma_{t} \sigma_{j}-2 \delta_{j i} \sigma_{t}+2 \delta_{j t} \sigma_{i}-2 \sigma_{i} \sigma_{t} \sigma_{j}-2 \delta_{j i} \sigma_{t}+2 \delta_{i t} \sigma_{j}-2 \sigma_{i} \sigma_{t} \sigma_{j}, \\
& =2 \delta_{j t} \sigma_{i}+2 \delta_{i t} \sigma_{j}-4 \delta_{j i} \sigma_{t} .
\end{aligned}
$$

Plugging in equation (B.6)

$$
\begin{aligned}
\mathcal{D}[\rho(t)] & =\frac{1}{2} \sum_{i, j=1}^{3} a_{i j}\left(4 i \rho_{0} \epsilon_{i j g} \sigma_{g}+\sum_{t=1}^{3}\left(2 \delta_{j t} \sigma_{i}+2 \delta_{i t} \sigma_{j}-4 \delta_{j i} \sigma_{t}\right) \rho_{t}\right) \\
& =\sum_{j, i=1}^{3} 2 i a_{i j} \rho_{0} \epsilon_{i j g} \sigma_{g}+\sum_{i, j=1}^{3}\left(a_{i j} \sigma_{i} \rho_{j}+a_{i j} \sigma_{j} \rho_{i}\right)-2 \sum_{i, t=1}^{3} a_{i i} \sigma_{t} \rho_{t} .
\end{aligned}
$$

Exchanging the indexes $j \leftrightarrow i$ in one of the terms of the second piece above and making use of the fact that $a_{i j}=\left(a_{j i}\right)^{*}$ (see equation (2.28))

$$
\begin{aligned}
\mathcal{D}[\rho(t)] & =\sum_{j, i=1}^{3} 2 i a_{i j} \rho_{0} \epsilon_{i j g} \sigma_{g}+\sum_{i, j=1}^{3}\left(a_{i j}+\left(a_{i j}\right)^{*}\right) \sigma_{i} \rho_{j}-2 \sum_{i, t=1}^{3} a_{i i} \sigma_{t} \rho_{t}, \\
& =\sum_{j, i=1}^{3} 2 i a_{i j} \rho_{0} \epsilon_{i j g} \sigma_{g}+\sum_{i, j=1}^{3} 2 \operatorname{Re}\left(a_{i j}\right) \sigma_{i} \rho_{j}-2 \sum_{i, t=1}^{3} a_{i i} \sigma_{t} \rho_{t} .
\end{aligned}
$$

Performing the summation explicitly and factorazing the terms in the basis $\left(\sigma_{0}, \sigma_{1}, \sigma_{2}, \sigma_{3}\right)$ we have (3.5). 


\section{APPENDIX C}

\section{THE CHECKER MAP FOR THE TWO- AND THREE-DIMENSIONAL DISSIPATOR}

In this appendix we show a tool we have developed, the Checker map. It is a map which relates the Kossakowski matrix and the Dissipator $\mathcal{D}$. This is useful to see how $\mathcal{D}$ changes depending on the terms we turn on in the Kossakoski matrix.

\section{C.1 Mapping the two-dimensional Dissipator}

The Checker map is represented by the figure below.

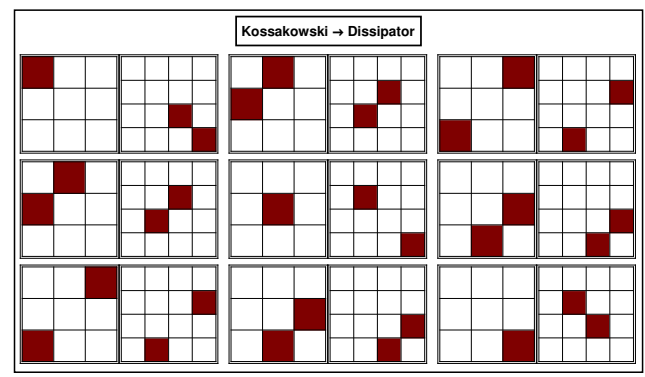

Figure C.1: The Checker map, a useful tool to relate the elements of th Kossakowski matrix with the Dissipator. 


\section{C.2 Mapping the three-dimensional Dissipator}

In three dimensions the Checker map is

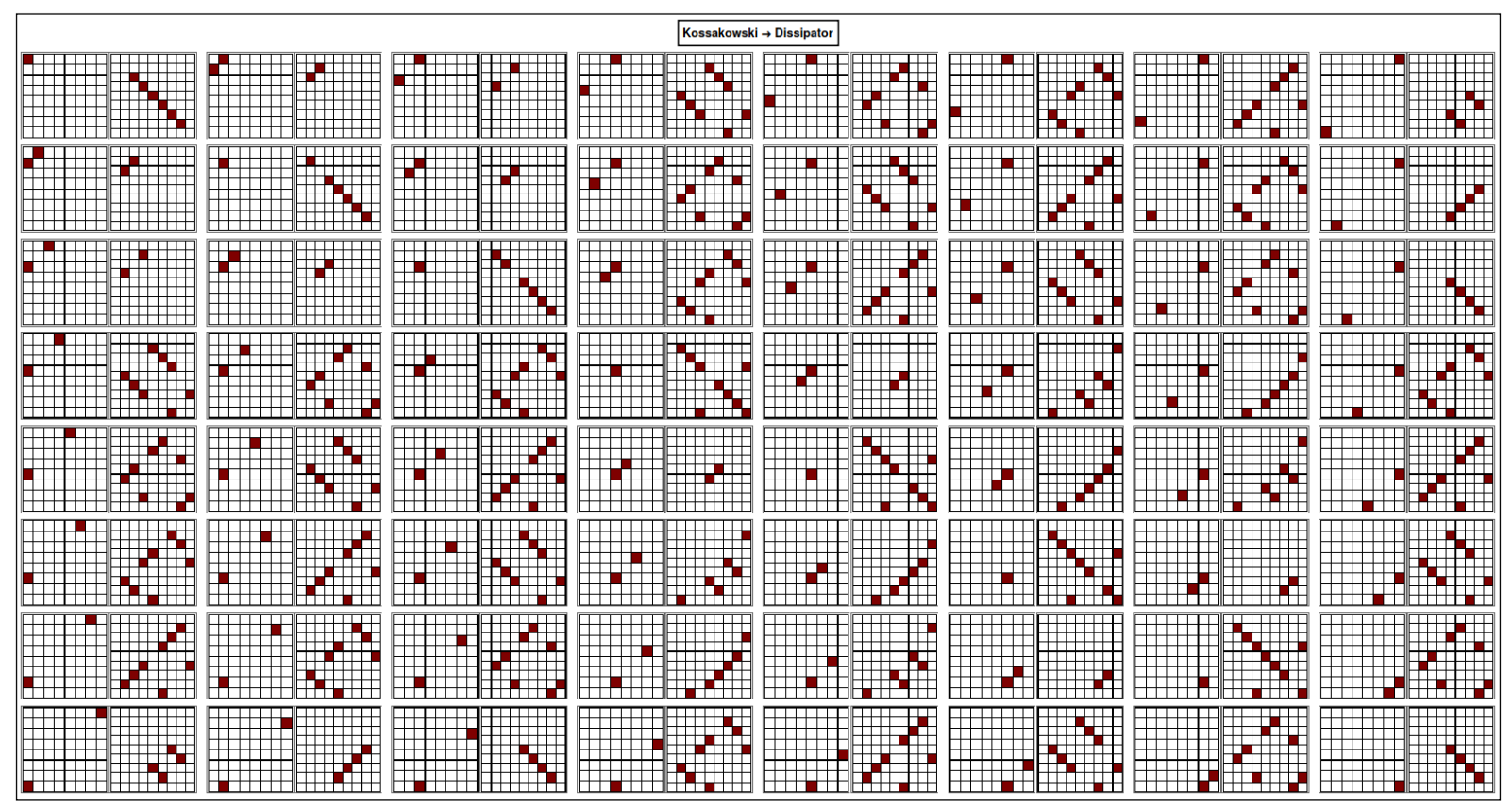

Figure C.2: Same as figure C.1, but for a three-dimensional system.

\section{C.3 Components of the three dimensional Dissipator}

The diagonal terms are

$$
\begin{aligned}
& \mathcal{D}_{22}=\frac{1}{2}\left(-4 a_{22}-4 a_{33}-a_{44}-a_{55}-a_{66}-a_{77}\right) \\
& \mathcal{D}_{33}=\frac{1}{2}\left(-4 a_{11}-4 a_{33}-a_{44}-a_{55}-a_{66}-a_{77}\right) \\
& \mathcal{D}_{44}=\frac{1}{2}\left(-4 a_{11}-4 a_{22}-a_{44}-a_{55}-a_{66}-a_{77}\right) \\
& \mathcal{D}_{55}=\frac{1}{2}\left(-a_{11}-a_{22}-a_{33}-2 \sqrt{3} a_{38}-4 a_{55}-a_{66}-a_{77}-3 a_{88}\right) \\
& \mathcal{D}_{66}=\frac{1}{2}\left(-a_{11}-a_{22}-a_{33}-2 \sqrt{3} a_{38}-4 a_{44}-a_{66}-a_{77}-3 a_{88}\right) \\
& \mathcal{D}_{77}=\frac{1}{2}\left(-a_{11}-a_{22}-a_{33}+2 \sqrt{3} a_{38}-a_{44}-a_{55}-4 a_{77}-3 a_{88}\right) \\
& \mathcal{D}_{88}=\frac{1}{2}\left(-a_{11}-a_{22}-a_{33}+2 \sqrt{3} a_{38}-a_{44}-a_{55}-4 a_{66}-3 a_{88}\right) \\
& \mathcal{D}_{99}=-\frac{3}{2}\left(a_{44}+a_{55}+a_{66}+a_{77}\right)
\end{aligned}
$$


Since the matrix is symmetric the other necessary elements are

$$
\begin{aligned}
& \mathcal{D}_{23}=2 a_{12} \\
& \mathcal{D}_{24}=2 a_{13} \\
& \mathcal{D}_{25}=\frac{1}{2}\left(a_{14}-3 a_{25}+3 a_{36}+\sqrt{3} a_{68}\right) \\
& \mathcal{D}_{47}=\frac{1}{2}\left(3 a_{14}+3 a_{25}+a_{36}-\sqrt{3} a_{68}\right) \\
& \mathcal{D}_{26}=\frac{1}{2}\left(a_{15}+3 a_{24}+3 a_{37}+\sqrt{3} a_{78}\right) \\
& \mathcal{D}_{48}=\frac{1}{2}\left(3 a_{15}-3 a_{24}+a_{37}-\sqrt{3} a_{78}\right) \\
& \mathcal{D}_{49}=\frac{\sqrt{3}}{2}\left(-a_{44}-a_{55}+a_{66}+a_{77}\right) \\
& \mathcal{D}_{27}=\frac{1}{2}\left(a_{16}+3 a_{27}-3 a_{34}+\sqrt{3} a_{48}\right) \\
& \mathcal{D}_{56}=2 a_{45} \\
& \mathcal{D}_{28}=\frac{1}{2}\left(a_{17}-3 a_{26}-3 a_{35}+\sqrt{3} a_{58}\right) \\
& \mathcal{D}_{57}=\frac{1}{2}\left(-2 \sqrt{3} a_{18}+a_{46}-3 a_{57}\right) \\
& \mathcal{D}_{29}=-\sqrt{3}\left(a_{46}+a_{57}\right) \\
& \mathcal{D}_{34}=2 a_{23} \\
& \mathcal{D}_{35}=\frac{1}{2}\left(3 a_{15}+a_{24}-3 a_{37}-\sqrt{3} a_{78}\right) \\
& \mathcal{D}_{36}=\frac{1}{2}\left(-3 a_{14}+a_{25}+3 a_{36}+\sqrt{3} a_{68}\right) \\
& \mathcal{D}_{37}=\frac{1}{2}\left(-3 a_{17}+a_{26}-3 a_{35}+\sqrt{3} a_{58}\right) \\
& \mathcal{D}_{38}=\frac{1}{2}\left(3 a_{16}+a_{27}+3 a_{34}-\sqrt{3} a_{48}\right) \\
& \mathcal{D}_{58}=\frac{1}{2}\left(2 \sqrt{3} a_{28}+a_{47}+3 a_{56}\right) \\
& \mathcal{D}_{59}=\frac{1}{2}\left(\sqrt{3} a_{16}-\sqrt{3} a_{27}+\sqrt{3} a_{34}+3 a_{48}\right) \\
& \mathcal{D}_{67}=\frac{1}{2}\left(-2 \sqrt{3} a_{28}+3 a_{47}+a_{56}\right) \\
& \mathcal{D}_{68}=\frac{1}{2}\left(-2 \sqrt{3} a_{18}-3 a_{46}+a_{57}\right) \\
& \mathcal{D}_{69}=\frac{1}{2}\left(\sqrt{3} a_{17}+\sqrt{3} a_{26}+\sqrt{3} a_{35}+3 a_{58}\right) \\
& \mathcal{D}_{39}=\sqrt{3}\left(a_{47}-a_{56}\right) \\
& \mathcal{D}_{45}=\frac{1}{2}\left(-3 a_{16}+3 a_{27}+a_{34}+\sqrt{3} a_{48}\right) \\
& \mathcal{D}_{46}=\frac{1}{2}\left(-3 a_{17}-3 a_{26}+a_{35}+\sqrt{3} a_{58}\right) \\
& \mathcal{D}_{78}=2 a_{67} \\
& \mathcal{D}_{79}=\frac{1}{2}\left(\sqrt{3} a_{14}+\sqrt{3} a_{25}-\sqrt{3} a_{36}+3 a_{68}\right) \\
& \mathcal{D}_{89}=\frac{1}{2}\left(\sqrt{3} a_{15}-\sqrt{3} a_{24}-\sqrt{3} a_{37}+3 a_{78}\right)
\end{aligned}
$$




\section{APPENDIX D}

\section{CALCULATION OF THE DISTANCE A NEUTRINO TRAVEL THROUGH EACH EARTH LAYER}

In this appendix we will derive the formula that connects the distance a neutrino travel through each matter layer in Earth with the zenith angle $\theta_{z}$.

\section{D.1 Possible paths travelled by a neutrino through the Earth}

In this appendix we will define the zenith angle $\theta_{z}$ to be zero for a neutrino crossing the Earth diameter just to simplify the calculations. This angle $\theta_{z}$ is related to the angle used in chapter [4], through the constraint

$$
\theta_{z}^{\mathrm{IC}}=\pi-\theta_{z}
$$

where IC stands for IceCube to remind that this is the orientation usually adopted by the collaboration.

\section{D.1.1 The boundaries angles}

First of all, it is necessary to define the zenith angles that define the layers boundaries (see figure D.1). By straightforward trigonometry we have that 


$$
\sin \theta_{z}^{i}=\frac{R_{i}}{R_{E}},
$$

where $R_{i}$ is the radius of the ith layer and $R_{E}$ the Earth radius.

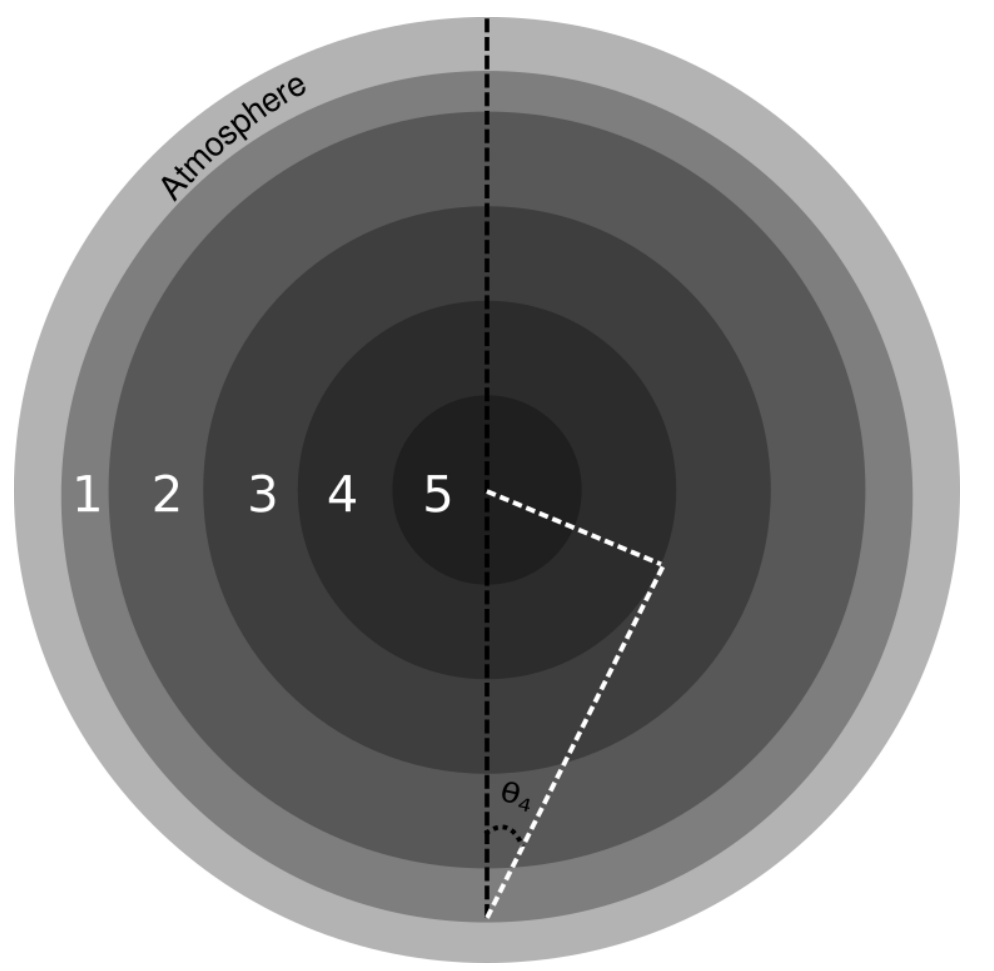

Figure D.1: Path defining boundary the angle of the fourth layer.

\section{D.1.2 First possibility}

The first possible path we will study is illustrated in figure D.2. In this first case, the neutrino travels through the distance

$$
L=2 \Delta x_{1}+\Delta x_{2}
$$

Since we are considering the Earth as a sphere, using trigonometry relations we have the constraint

$$
L=2 R_{E} \cos \theta_{z}
$$

Considering the right triangle formed by the white continuous line, the white dashed line and the black dashed line we can find the size of the white line $S$ 


$$
S=R_{E} \sin \theta_{z}
$$

Now considering the right triangle formed by the same white lines used before, but with hypotenuse given by the red dashed line we can use the Pythagorean theorem to find $\Delta x_{2}$

$$
\Delta x_{2}=2 \sqrt{R_{2}^{2}-R_{E}^{2} \sin ^{2} \theta_{z}}
$$

From the constraint (D.4) we can find $\Delta x_{1}$

$$
\Delta x_{1}=R_{E} \cos \theta_{z}-\sqrt{R_{2}^{2}-R_{E}^{2} \sin ^{2} \theta_{z}}
$$

The path travelled through the atmosphere is discussed in the last subsection

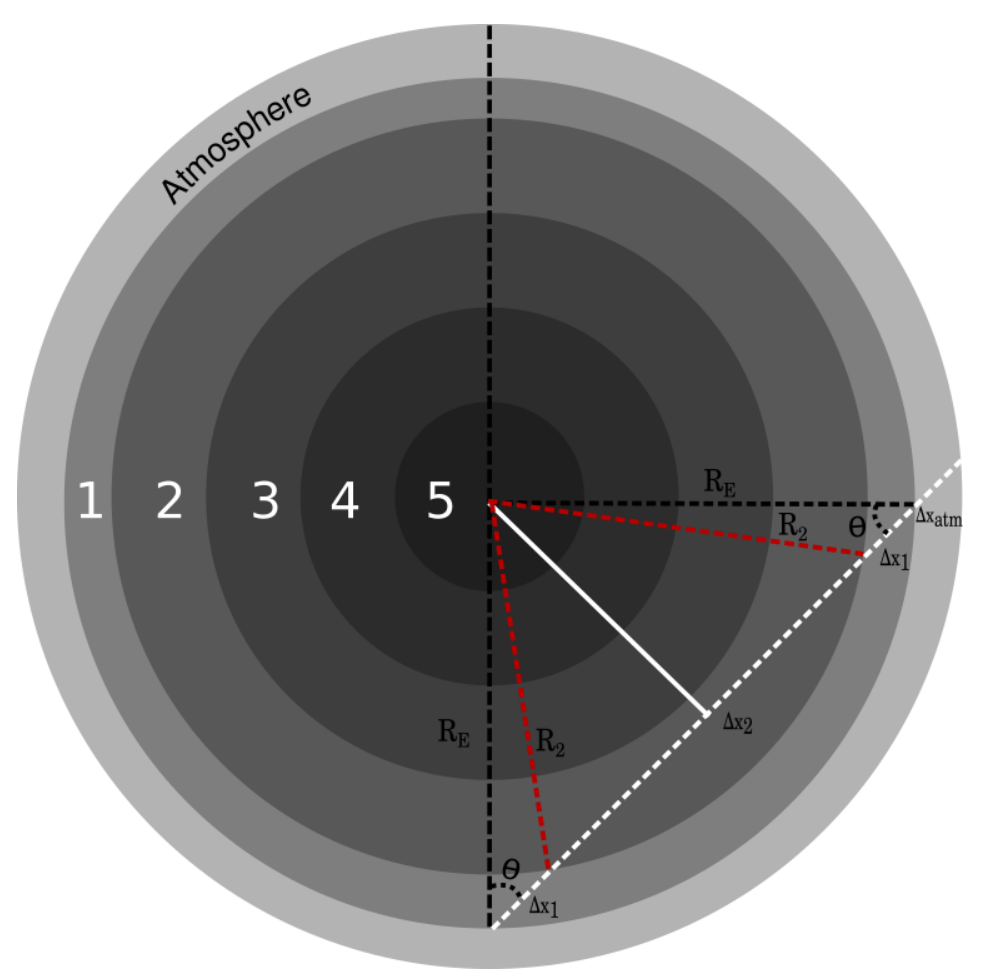

Figure D.2: First possible scenario.

\section{D.1.3 Second possibility}

This scenario consists of a neutrino crossing only one layer and the atmosphere 


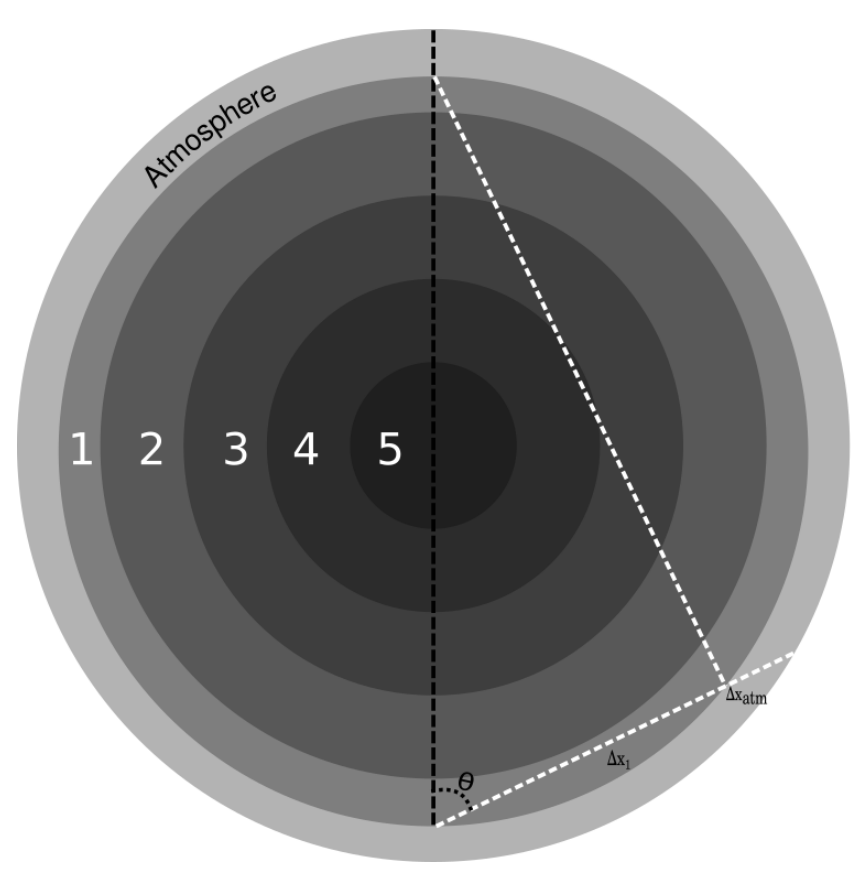

Figure D.3: Second possible scenario.

The right triangle implies that

$$
\Delta x_{1}=2 R_{E} \cos \theta_{z}
$$

\section{D.1.4 Third possibility}

The last case is useful to make the result general and it is depicted in figure D.4. We have three different right triangles formed by the dashed and continuous straight lines and the red, blue, black dashed lines, respectively. We can work from the inside to outside, the length $\Delta x_{3}$ can be found analogously to (D.6)

$$
\Delta x_{3}=2 \sqrt{R_{3}^{2}-R_{E}^{2} \sin ^{2} \theta_{z}}
$$

Using the second inner triangle we can find $\Delta x_{2}$ in this case

$$
\Delta x_{2}=\sqrt{R_{2}^{2}-R_{E}^{2} \sin ^{2} \theta_{z}}-\sqrt{R_{3}^{2}-R_{E}^{2} \sin ^{2} \theta_{z}} .
$$

For the outermost layer

$$
\Delta x_{1}=R_{E} \cos \theta_{z}-\sqrt{R_{2}^{2}-R_{E} \sin ^{2} \theta_{z}}
$$




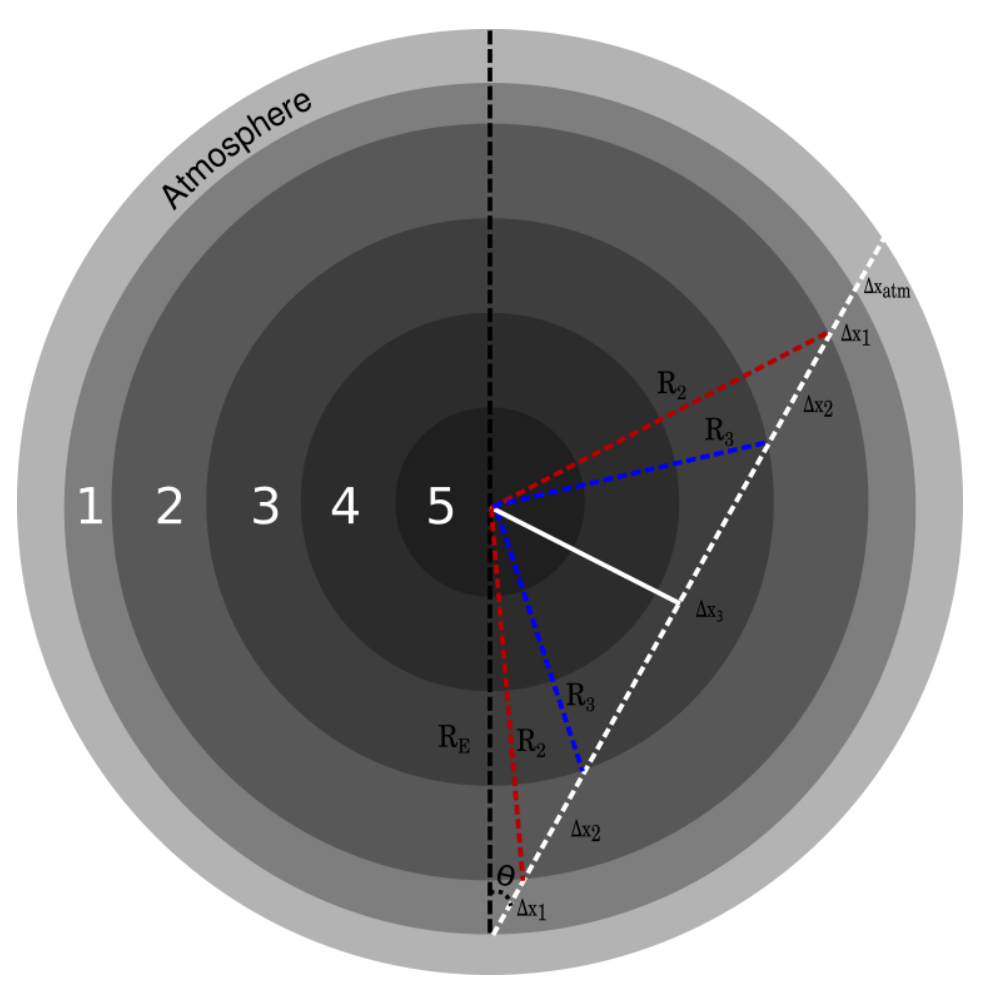

Figure D.4: Third possible scenario.

So the pattern is the following:

(a) In the innermost layer ${ }^{1}$ it will travel a distance

$$
\Delta x_{i}=2 \sqrt{R_{i}-R_{E}^{2} \sin ^{2} \theta_{z}}
$$

where $i$ is the index labeling the innermost layer.

(b) In the layers (labelled $j$ ) between the innermost and outermost layers it travels a distance

$$
\Delta x_{j}=\sqrt{R_{j}^{2}-R_{E}^{2} \sin ^{2} \theta_{z}}-\sqrt{R_{j+1}^{2}-R_{E}^{2} \sin \theta_{z}^{2}},
$$

where $j=2, \ldots, i-1$.

(c) In the outermost layer the distance travelled is

$$
\Delta x=R_{E} \cos \theta_{z}-\left(R_{2}^{2}-R_{E}^{2} \sin ^{2} \theta_{z}\right)
$$

\footnotetext{
${ }^{1}$ The innermost layer depends on the path the neutrino goes through. This information is encoded in the boundaries angles.
} 


\section{D.1.5 Distance travelled through the atmosphere}

For completeness we include here also the derive distance travelled through the atmosphere.

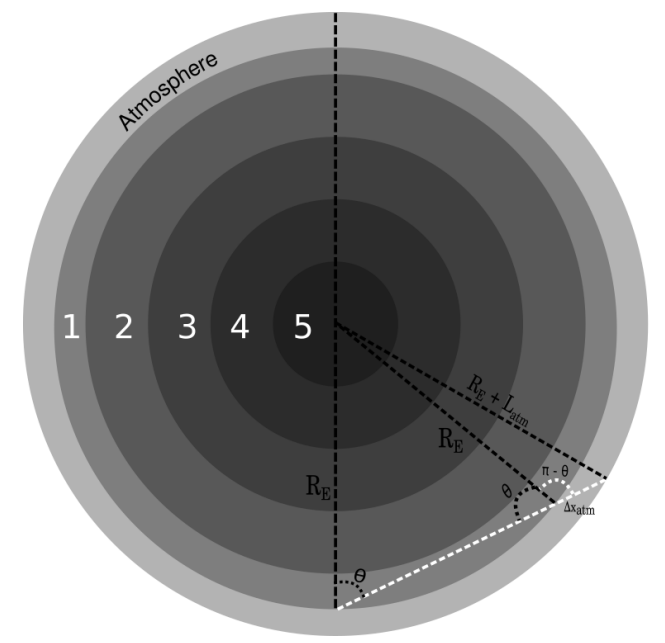

(a) Neutrino crossing the Earth and the atmosphere.

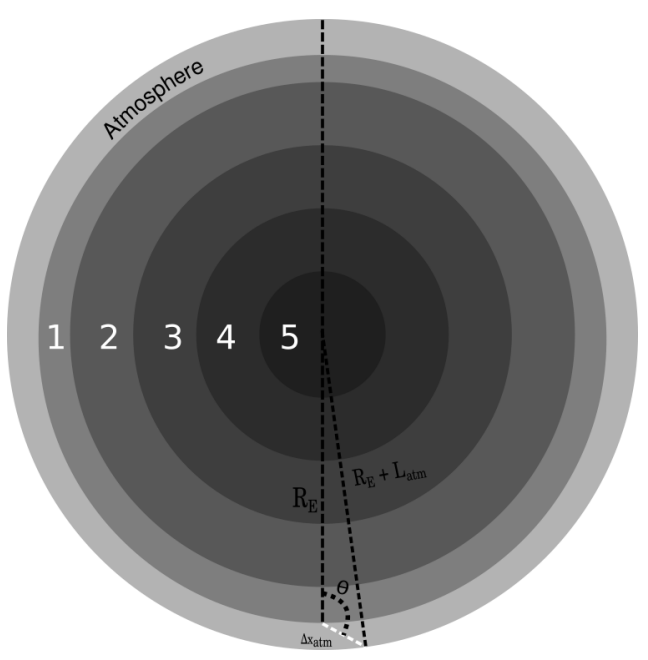

(b) Neutrino crossing only the atmosphere.

Figure D.5: Possible paths a neutrino can travel in the Earth atmosphere.

We have two distinct cases illustrated in the figure above. Considering that the orthogonal distance between the atmosphere and the first layer is $L_{a t m}$ and using the law of cosines we can find for figure D.5(a)

$$
\Delta x_{\mathrm{atm}}=\frac{1}{2}\left(-2 R_{E} \cos \theta_{z}+\sqrt{2} \sqrt{2 L_{\mathrm{atm}}^{2}+4 L_{\mathrm{atm}} R_{E}+R_{E}^{2}\left(1+\cos 2 \theta_{z}\right)}\right)
$$

and for figure D.5(b)

$$
\Delta x_{\mathrm{atm}}=\frac{1}{2}\left(2 R_{E} \cos \theta_{z}+\sqrt{2} \sqrt{2 L_{\mathrm{atm}}^{2}+4 L_{\mathrm{atm}} R_{E}+R_{E}^{2}\left(1+\cos 2 \theta_{z}\right)}\right) .
$$




\section{BIBLIOGRAPHY}

[1] W. Pauli. "Dear radioactive ladies and gentlemen". In: Phys. Today 31N9 (1978), p. 27.

[2] A. Franklin. Are there really neutrinos? An evidential history. CRC Press, Nov. 2000. ISBN: 978-0-7382-0265-5.

[3] J. Chadwick. "The intensity distribution in the magnetic spectrum of beta particles from radium (B + C)". In: Verh. Phys. Gesell. 16 (1914), pp. 383-391.

[4] J. Chadwick. "Possible Existence of a Neutron". In: Nature 129 (1932), p. 312. DOI: $10.1038 / 129312 \mathrm{a} 0$.

[5] F. Close. Small Things and Nothing. OUP Oxford, 2014. ISBN: 9780191025433. URL: https://books.google.com.au/books?id=PFP1AgAAQBAJ.

[6] T.D. Lee and Chen-Ning Yang. "Parity Nonconservation and a Two Component Theory of the Neutrino". In: Phys. Rev. 105 (1957). Ed. by G. Feinberg, pp. 1671-1675. DOI: 10.1103/PhysRev.105.1671.

[7] C.L. Cowan et al. "Detection of the free neutrino: A Confirmation". In: Science 124 (1956), pp. 103-104. DOI: 10.1126/science.124.3212.103.

[8] F. Reines and C.L. Cowan. "A Proposed experiment to detect the free neutrino". In: Phys. Rev. 90 (1953), pp. 492-493. DOI: 10.1103/PhysRev.90.492.

[9] J.N. Bahcall. Neutrino Astrophysics. Cambridge University Press, 1989. ISBN: 9780521379755. URL: https: / / books.google.com.br/books?id=8GIP7uNMhlsC. 
[10] A. Dar, G. Eilam, and M. Gronau. Neutrino 94: Proceedings of the 16th International Conference on Neutrino Physics and Astrophysics, Eilat, Israel, 29 May - 3 June 1994. Elsevier Science, 2016. ISBN: 9781483278070. URL: https : / / books . google. com.br/books? id=WAxcDAAAQBAJ.

[11] J. N. Abdurashitov et al. "Measurement of the Solar Neutrino Capture Rate by SAGE and Implications for Neutrino Oscillations in Vacuum". In: Physical Review Letters 83.23 (Dec. 1999), pp. 4686-4689. ISSN: 1079-7114. DOI: 10.1103 / physrevlett. 83.4686. URL: http://dx.doi.org/10.1103/PhysRevLett.83.4686.

[12] M. Altmann et al. "GNO solar neutrino observations: results for GNO I". In: Physics Letters B 490.1-2 (Sept. 2000), pp. 16-26. ISSN: 0370-2693. DOI: $10.1016 /$ s 0370 2693 (00) 00915-1. URL: http:// dx . doi . org/10 .1016/s0370 $2693(00) 00915-1$.

[13] P. Anselmann et al. "Solar neutrinos observed by GALLEX at Gran Sasso." In: Phys. Lett. B 285 (1992), pp. 376-389. DOI: $10.1016 / 0370-2693$ (92) 91521-A.

[14] S. Fukuda et al. "SolarB8and hep Neutrino Measurements from 1258 Days of SuperKamiokande Data”. In: Physical Review Letters 86.25 (June 2001), pp. 5651-5655. ISSN: 1079-7114. DOI: 10.1103 / physrevlett.86.5651. URL: http: / / dx . doi.org/10.1103/PhysRevLett.86.5651.

[15] Y. Fukuda et al. "Solar neutrino data covering solar cycle 22". In: Phys. Rev. Lett. 77 (1996), pp. 1683-1686. DOI: 10 .1103/PhysRevLett. 77 .1683.

[16] K. Abe et al. "Measurement of Atmospheric Neutrino Flux Consistent with Tau Neutrino Appearance". In: Phys. Rev. Lett. 97 (17 Oct. 2006), p. 171801. DOI: $10.1103 /$ PhysRevLett . 97 . 171801. URL: https : / / link . aps . org/doi / 10 . 1103 /PhysRevLett.97.171801.

[17] K. Abe et al. "Search for matter-dependent atmospheric neutrino oscillations in SuperKamiokande”. In: Phys. Rev. D 77 (5 Mar. 2008), p. 052001. DOI: 10.1103 / Phys RevD . 77.052001. URL: https:// link.aps .org/doi/10 .1103/PhysRevD . 77.052001.

[18] W.W.M. Allison et al. "Measurement of the atmospheric neutrino flavor composition in Soudan-2". In: Phys. Lett. B 391 (1997), pp. 491-500. DOI: 10 . 1016 / S 0370 2693 (96)01609-7. arXiv: hep-ex/9611007. 
[19] R. Becker-Szendy et al. "Electron- and muon-neutrino content of the atmospheric flux". In: Phys. Rev. D 46 (9 Nov. 1992), pp. 3720-3724. DOI: 10.1103 / Phys RevD . 46. 3720. URL: https://link.aps.org/doi/10.1103/PhysRevD.46.3720.

[20] Y. Fukuda et al. "Atmospheric muon-neutrino / electron-neutrino ratio in the multiGeV energy range". In: Phys. Lett. B 335 (1994), pp. 237-245. DOI: 10 . $1016 / 0370-$ $2693(94) 91420-6$.

[21] Y. Fukuda et al. "Evidence for Oscillation of Atmospheric Neutrinos". In: Phys. Rev. Lett. 81 (8 Aug. 1998), pp. 1562-1567. DOI: 10.1103 / PhysRevLett. 81.1562. URL: https://link.aps.org/doi/10.1103/PhysRevLett.81.1562.

[22] K.S. Hirata et al. "Observation of a small atmospheric muon-neutrino / electron-neutrino ratio in Kamiokande". In: Phys. Lett. B 280 (1992), pp. 146-152. DOI: 10 . $1016 /$ $0370-2693(92) 90788-6$.

[23] Q. R. Ahmad et al. "Measurement of the Rate ofve $+d \rightarrow p+p+e I n t e r a c t i o n s$ Produced byB8Solar Neutrinos at the Sudbury Neutrino Observatory". In: Physical Review Letters 87.7 (July 2001). ISSN: 1079-7114. DOI: 10.1103 / physrevlett . 87.071301. URL: http://dx.doi.org/10.1103/PhysRevLett.87.071301.

[24] Y. Abe et al. "Indication of Reactor $\bar{\nu}_{e}$ Disappearance in the Double Chooz Experiment”. In: Phys. Rev. Lett. 108 (13 Mar. 2012), p. 131801. DoI: 10.1103 / Phy sRevLett. 108.131801. URL: https: / / ink.aps.org/doi/10.1103/PhysRevLett. 108.131801 .

[25] J. K. Ahn et al. "Observation of Reactor Electron Antineutrinos Disappearance in the RENO Experiment”. In: Phys. Rev. Lett. 108 (19 May 2012), p. 191802. DOI: 10 . 1103 / PhysRevLett.108.191802. URL: https:/ / link.aps.org/doi/ 10.1103 /PhysRevLett.108.191802.

[26] F. P. An et al. "Observation of Electron-Antineutrino Disappearance at Daya Bay". In: Phys. Rev. Lett. 108 (17 Apr. 2012), p. 171803. DoI: 10 . 1103 / PhysRevLett . 108.171803. URL: https://link.aps.org/doi/10.1103/PhysRevLett. 108.171803 .

[27] T. Araki et al. "Measurement of Neutrino Oscillation with KamLAND: Evidence of Spectral Distortion”. In: Phys. Rev. Lett. 94 (8 Mar. 2005), p. 081801. DOI: 10.1103 / PhysRevLett .94 .081801. uRL: https : / / link .aps . org/doi / 10 . 1103 /PhysRevLett.94.081801. 
[28] K. Eguchi et al. "First Results from KamLAND: Evidence for Reactor Antineutrino Disappearance". In: Phys. Rev. Lett. 90 (2 Jan. 2003), p. 021802. DOI: 10.1103 / PhysRevLett .90 .021802. uRl: https : / / link . aps . org/doi/10 . 1103 /PhysRevLett.90.021802.

[29] K. Abe et al. "Indication of Electron Neutrino Appearance from an Accelerator-Produced Off-Axis Muon Neutrino Beam". In: Phys. Rev. Lett. 107 (4 July 2011), p. 041801. DOI: 10 .1103/PhysRevLett.107.041801. URL: https: / / link .aps .org / doi/10.1103/PhysRevLett.107.041801.

[30] P. Adamson et al. "Improved Search for Muon-Neutrino to Electron-Neutrino Oscillations in MINOS”. In: Phys. Rev. Lett. 107 (18 Oct. 2011), p. 181802. DOI: $10.1103 /$ PhysRevLett . 107 . 181802. URL: https : / / link . aps . org/doi/10 . 1103 /PhysRevLett.107.181802.

[31] P. Adamson et al. "Measurement of Neutrino Oscillations with the MINOS Detectors in the NuMI Beam”. In: Phys. Rev. Lett. 101 (13 Sept. 2008), p. 131802. DOI: $10.1103 /$ PhysRevLett . 101 . 131802. URL: https : / / link . aps . org/doi / 10 . 1103 /PhysRevLett.101.131802.

[32] M. H. Ahn et al. "Measurement of neutrino oscillation by the K2K experiment". In: Phys. Rev. D 74 (7 Oct. 2006), p. 072003. DOI: 10.1103 / PhysRevD . 74.072003. URL: https://link.aps.org/doi/10.1103/PhysRevD.74.072003.

[33] E. Aliu et al. "Evidence for Muon Neutrino Oscillation in an Accelerator-Based Experiment”. In: Phys. Rev. Lett. 94 (8 Mar. 2005), p. 081802. DOI: 10.1103 / P hy s RevLett. 94.081802. URL: https://link.aps.org/doi/10.1103/PhysRevLett. 94.081802 .

[34] Andrea Donini, Sergio Palomares-Ruiz, and Jordi Salvado. "Neutrino tomography of Earth". In: Nature Physics 15.1 (Nov. 2018), pp. 37-40. ISSN: 1745-2481. DOI: 10 . 1038 / s41567-018-0319-1. URL: http://dx . doi .org/10 . 1038/ s41567-018-0319-1.

[35] A. Pais and O. Piccioni. "Note on the Decay and Absorption of the Theta0". In: Phys. Rev. 100 (1955), pp. 1487-1489. DOI: 10.1103 /PhysRev. 100.1487.

[36] B. Pontecorvo. "Mesonium and anti-mesonium". In: Sov. Phys. JETP 6 (1957), p. 429. 
[37] Ziro Maki, Masami Nakagawa, and Shoichi Sakata. "Remarks on the Unified Model of Elementary Particles". In: Progress of Theoretical Physics 28.5 (Nov. 1962), pp. 870880. ISSN: 0033-068X. DOI: 10 .1143/PTP .28 . 870. eprint: https: / / academic . oup.com/ptp/article-pdf/28/5/870/5258750/28-5-870.pdf. URL: https://doi.org/10.1143/PTP.28.870.

[38] B.T. Cleveland et al. "Measurement of the solar electron neutrino flux with the Homestake chlorine detector”. In: Astrophys. J. 496 (1998), pp. 505-526. DOI: 10.1086 / 305343.

[39] R. Davis. "A review of the Homestake solar neutrino experiment". In: Prog. Part. Nucl. Phys. 32 (1994), pp. 13-32. DOI: $10.1016 / 0146-6410$ (94) $90004-3$.

[40] W. Hampel et al. "GALLEX solar neutrino observations: Results for GALLEX IV". In: Phys. Lett. B 447 (1999), pp. 127-133. DOI: 10.1016/S0370-2693 (98) 015792.

[41] K.S. Hirata et al. "Observation of B-8 Solar Neutrinos in the Kamiokande-II Detector". In: Phys. Rev. Lett. 63 (1989), p. 16. DOI: $10.1103 /$ PhysRevLett. 63.16.

[42] Y. Fukuda et al. "Measurements of the Solar Neutrino Flux from Super-Kamiokande's First 300 Days”. In: Physical Review Letters 81.6 (Aug. 1998), pp. 1158-1162. ISSN: 1079-7114. DoI: 10.1103 / physrevlett.81.1158. URL: http://dx.doi . org/10.1103/PhysRevLett.81.1158.

[43] E Kh Akhmedov and A Yu Smirnov. "Paradoxes of neutrino oscillations". In: (2009). arXiv: arXiv:0905.1903v2.

[44] Evgeny K. Akhmedov and Joachim Kopp. "Neutrino oscillations: Quantum mechanics vs. quantum field theory”. In: Journal of High Energy Physics (2010). ISSN: 10298479. DOI: 10.1007 / JHEP 04 (2010)008. arXiv: 1001.4815.

[45] Mikael Beuthe. "Oscillations of neutrinos and mesons in quantum field theory". In: Physics Reports 375.2-3 (Feb. 2003), pp. 105-218. ISSN: 0370-1573. DOI: 10.1016 / s0370-1573 (02) 00538-0. URL: http://dx.doi.org/10.1016/s0370$1573(02) 00538-0$.

[46] Boris Kayser. "On the Quantum Mechanics of Neutrino Oscillation”. In: Phys. Rev. D 24 (1981), p. 110. DOI: 10.1103 /PhysRevD.24.110. 
[47] C. Giunti, C. W. Kim, and U. W. Lee. "When do neutrinos really oscillate? Quantum mechanics of neutrino oscillations". In: Phys. Rev. D 44 (11 Dec. 1991), pp. 3635-3640. DOI: 10.1103 /PhysRevD .44.3635. URL: https://link.aps .org/doi/ $10.1103 /$ PhysRevD . 44.3635.

[48] Evgeny Kh. Akhmedov and Alina Wilhelm. "Quantum field theoretic approach to neutrino oscillations in matter". In: Journal of High Energy Physics 2013.1 (Jan. 2013). ISSN: 1029-8479. DOI: $10.1007 /$ jhep 01 (2013) 165. URL: http: / / dx . doi . org/10.1007/JHEPO1 (2013) 165.

[49] Evgeny Akhmedov. "Quantum mechanics aspects and subtleties of neutrino oscillations”. In: (2019). arXiv: 1901.05232 [hep-ph].

[50] S. Nussinov. "Solar Neutrinos and Neutrino Mixing". In: Phys. Lett. B 63 (1976), pp. 201-203. DOI: $10.1016 / 0370-2693$ (76) 90648-1.

[51] C Giunti. "The Phase of Neutrino Oscillations". In: Physica Scripta 67.1 (Jan. 2003), pp. 29-33. ISSN: 1402-4896. DOI: 10 . 1238 / physica.regular. 067 a00029. URL: http://dx.doi.org/10.1238/Physica.Regular.067a00029.

[52] C Giunti, C W Kim, and U W Lee. "When do neutrinos cease to oscillate?" In: 9tember (1997). arXiv: $9709494 \mathrm{v}$ [arXiv:hep-ph].

[53] Carlo Giunti, Sezione Torino, and Fisica Teorica. "Coherence and Wave Packets in Neutrino Oscillations Carlo Giunti”. In: October (2003), pp. 1-21. arXiv: 0302026v3 [arXiv:hep-ph].

[54] C Giunti. "Neutrino Wave Packets in Quantum Field Theory”. In: (2002). arXiv: 0205014 v2 [arXiv:hep-ph].

[55] W Grimus. "Revisiting the quantum field theory of neutrino oscillations in vacuum". In: (2020), pp. 1-26. arXiv: arXiv:1910.13776v3.

[56] W Grimus and P Stockinger. "arXiv : hep-ph / 9603430v1 28 Mar 1996 Real Oscillations of Virtual Neutrinos". In: (2018). arXiv: $9603430 \mathrm{v}$ [ [arXiv: hep-ph] .

[57] W Grimus, P Stockinger, and S Mohanty. "The field-theoretical approach to coherence in neutrino oscillations". In: 9tember (1998), pp. 1-18. arXiv: 9807442 v2 [arXiv: hep-ph] .

[58] D V Naumov. "On the Theory of Wave Packets". In: (), pp. 1-20. arXiv: arXiv : $1309.1717 \mathrm{v} 1$. 
[59] D V Naumov and V A Naumov. "A diagrammatic treatment of neutrino oscillations". In: Journal of Physics G: Nuclear and Particle Physics 37.10 (Aug. 2010), p. 105014. DOI: 10 .1088/0954-3899/37/10/105014. URL: https://doi.org/10. $1088 \div 2 \mathrm{~F} 0954-3899 \div 2 \mathrm{~F} 37 \div 2 \mathrm{~F} 10 \div 2 \mathrm{~F} 105014$.

[60] D.V. Naumov and V.A. Naumov. "Quantum Field Theory of Neutrino Oscillations". In: Phys. Part. Nucl. 51.1 (2020), pp. 1-106. DOI: 10.1134 / S1 063779620010050.

[61] Vadim A Naumov and Dmitry S Shkirmanov. "9tember 7, 2018 3:14 WSPC/INSTRUCTION FILE AWP”. In: (2018), pp. 1-18. arXiv: arXiv:1409.4669v2.

[62] Joachim Kopp and Manfred Lindner. "Oscillations of $\mathbf{M}^{*}$ ossbauer neutrinos". In: 2 (2018), pp. 1-31. arXiv: arXiv:0802.2513v2.

[63] D V Naumov, V A Naumov, and D S Shkirmanov. "Quantum Field Theoretical Description of Neutrino Oscillations and Reactor Antineutrino Anomaly 1, 2”. In: 48.6 (2017), pp. 1007-1010. DOI: 10.1134/S1063779617060430.

[64] M.D. Schwartz. Quantum Field Theory and the Standard Model. Quantum Field Theory and the Standard Model. Cambridge University Press, 2014. ISBN: 9781107034730. URL: https: / / books.google.com.br/books?id=HbdEAgAAQBAJ.

[65] W. Grimus and P. Stockinger. "Real oscillations of virtual neutrinos". In: Physical Review D 54.5 (Sept. 1996), pp. 3414-3419. ISSN: 1089-4918. DOI: 10.1103 / physrevd. 54.3414. URL: http://dx.doi.org/10.1103/PhysRevD.54.3414.

[66] J. Rich. "The Quantum mechanics of neutrino oscillations". In: Phys. Rev. D 48 (1993), pp. 4318-4325. DOI: 10.1103/PhysRevD . 48.4318.

[67] J. Linder. Neutrino matter potentials induced by Earth. 2005. arXiv: hep-ph / 0504264 [hep-ph].

[68] A. Yu. Smirnov. The MSW effect and Solar Neutrinos. 2003. arXiv: hep-ph / 0305106 [hep-ph].

[69] V. Barger, D. Marfatia, and K. Whisnant. "Confronting mass-varying neutrinos with MiniBooNE”. In: Phys. Rev. D 73 (2006), p. 013005. DoI: 10.1103 / PhysRevd . 73.013005. arXiv: hep-ph/0509163.

[70] Y. Farzan and M. Tortola. "Neutrino oscillations and Non-Standard Interactions". In: Front. in Phys. 6 (2018), p. 10. DOI: 10.3389/ fphy . 2018.00010 . arXiv: 1710. 09360 [hep-ph]. 
[71] Dmitry V. Naumov. “The Sterile Neutrino: A short introduction”. In: EPJ Web Conf. 207 (2019). Ed. by C. Spiering, p. 04004. DoI: 10 . 1051 / ep jconf / 201920704004. arXiv: 1901.00151 [hep-ph] .

[72] S. W. Hawking. "Breakdown of predictability in gravitational collapse". In: Phys. Rev. D 14 (10 Nov. 1976), pp. 2460-2473. DOI: 10.1103 / Phy sRevD . 14 . 2460 . URL: https://link.aps.org/doi/10.1103/PhysRevD.14.2460.

[73] B.S. Dewitt and N. Graham. The Many Worlds Interpretation of Quantum Mechanics. Princeton Series in Physics. Princeton University Press, 2015. ISBN: 9781400868056. URL: https://books.google.com.br/books?id=qPF 9BgAAQBAJ.

[74] A. Einstein, B. Podolsky, and N. Rosen. "Can Quantum-Mechanical Description of Physical Reality Be Considered Complete?" In: Phys. Rev. 47 (10 May 1935), pp. 777780. DOI: 10 . 1103 / PhysRev . 47 . 777. URL: https : / / link .aps . org / doi/10.1103/PhysRev.47.777.

[75] L.E. Ballentine. Quantum Mechanics: A Modern Development. World Scientific, 1998. ISBN: 9789810241056. URL: https: / / books . google.com.br/books?id= SHJRFHZ1rYsC.

[76] Á. Rivas and S.F. Huelga. Open Quantum Systems: An Introduction. SpringerBriefs in Physics. Springer Berlin Heidelberg, 2011. ISBN: 9783642233548. URL: https: //books.google.com.br/books?id=FGCuYsIZAAOC.

[77] H.P. Breuer et al. The Theory of Open Quantum Systems. Oxford University Press, 2002. ISBN: 9780198520634. URL: https: / / books . google.com.br/books? id= OYx5VzaMYm8C.

[78] G. Lindblad. "On the generators of quantum dynamical semigroups". In: Comm. Math. Phys. 48.2 (1976), pp. 119-130. URL: https : / / projecteuclid.org: 443 / euclid.cmp/1103899849.

[79] Vittorio Gorini, Andrzej Kossakowski, and E. C. G. Sudarshan. "Completely positive dynamical semigroups of N-level systems". In: Journal of Mathematical Physics 17.5 (1976), pp. 821-825. DOI: 10 . $1063 / 1$.522979. eprint: https : / / aip . scitation.org/doi/pdf/10.1063/1.522979. URL: https: / / aip. scitation.org/doi/abs/10.1063/1.522979.

[80] A Kossakowski. "Quantum statistical mechanics of non-Hamiltonian systems". In: Rep. Math. Phys., v. 3, no. 4, pp. 247-274 (Dec. 1972). DOI: $10.1016 / 0034-4877$ (72) $90010-9$. 
[81] K. Kraus et al. States, effects, and operations: fundamental notions of quantum theory : lectures in mathematical physics at the University of Texas at Austin. Lecture notes in physics. Springer-Verlag, 1983. ISBN: 9780387127323. URL: https : / / books . google.com.br/books?id=fRBBAQAAIAAJ.

[82] Fabio Benatti and roberto Floreanini. "Complete positivity and the neutral kaon system”. In: Banach Center Publications 43.1 (1998), pp. 71-85. ISSN: 0137-6934. DOI: $10.4064 /-43-1-71-85$.

[83] Fabio Benatti and Heide Narnhofer. "Entropy behaviour under completely positive maps". In: Letters in Mathematical Physics 15 (Jan. 1988), pp. 325-334. DOI: 10 . 1007 / BF00419590.

[84] Fabio Benatti and Roberto Floreanini. "Open system approach to neutrino oscillations". In: Journal of High Energy Physics 2000.02 (Feb. 2000), pp. 032-032. ISSN: 10298479. DOI: 10 .1088/1126-6708/2000/02/032. URL: http:// dx.doi . org/10.1088/1126-6708/2000/02/032.

[85] J.A. Carpio, E. Massoni, and A.M. Gago. "Revisiting quantum decoherence for neutrino oscillations in matter with constant density”. In: Physical Review D 97.11 (June 2018). ISSN: 2470-0029. DOI: 10.1103/physrevd.97 .115017. URL: http : / / dx . doi.org/10.1103/PhysRevD.97.115017.

[86] R. L.N. Oliveira and M. M. Guzzo. "Quantum dissipation in vacuum neutrino oscillation”. In: European Physical Journal C 69.3 (2010), pp. 493-502. ISSN: 14346044. DOI: $10.1140 / \mathrm{epjc} / \mathrm{s} 10052-010-1388-1$.

[87] Ara Ioannisian et al. Analytical description of CP violation in oscillations of atmospheric neutrinos traversing the Earth. 2020. arXiv: 2005.07719 [hep-ph] .

[88] A.M. Dziewonski and D.L. Anderson. "Preliminary reference earth model". In: Phys. Earth Planet. Interiors 25 (1981), pp. 297-356. DOI: $10.1016 / 0031-9201$ (81) $90046-7$.

[89] Ivan Esteban et al. "Global analysis of three-flavour neutrino oscillations: synergies and tensions in the determination of $\theta 23, \delta \mathrm{CP}$, and the mass ordering”. In: Journal of High Energy Physics 2019.1 (Jan. 2019). ISSN: 1029-8479. DOI: 10.1007 / jhep 01 (2019) 106. URL: http://dx.doi.org/10.1007/JHEP01 (2019)106.

[90] K. Abe et al. Constraint on the Matter-Antimatter Symmetry-Violating Phase in Neutrino Oscillations. 2019. arXiv: 1910.03887 [hep-ex ] . 
[91] M.G. Aartsen et al. "Neutrino oscillation studies with IceCube-DeepCore". In: Nucl. Phys. B 908 (2016), pp. 161-177. DOI: $10.1016 /$ j . nuclphys.b . 2016.03 .028$.

[92] M. G. Aartsen et al. "Measurement of atmospheric tau neutrino appearance with IceCube DeepCore". In: Physical Review D 99.3 (Feb. 2019). ISSN: 2470-0029. DOI: 10 . 1103 / physrevd.99.032007. URL: http://dx.doi .org/10.1103/ PhysRevD.99.032007.

[93] Martin Leuermann. “Testing the neutrino mass ordering with IceCube DeepCore”. PhD thesis. RWTH Aachen U., 2018. DOI: 10 .18154/RWTH-2018-231554.

[94] M. G. Aartsen et al. "Searches for Sterile Neutrinos with the IceCube Detector". In: Physical Review Letters 117.7 (Aug. 2016). ISSN: 1079-7114. DOI: 10.1103 / physrevlett . 117.071801. URL: http: / / dx.doi.org/10.1103/PhysRevLett.117. 071801.

[95] M. G. Aartsen et al. "Development of an analysis to probe the neutrino mass ordering with atmospheric neutrinos using three years of IceCube DeepCore data". In: The European Physical Journal C 80.1 (Jan. 2020). ISSN: 1434-6052. DOI: 10.1140 / ep jc/ s10052-019-7555-0. URL: http:// dx . doi .org/10.1140/epjc/ s10052-019-7555-0.

[96] M.G. Aartsen et al. "Measurement of Atmospheric Neutrino Oscillations at 6-56 GeV with IceCube DeepCore”. In: Phys. Rev. Lett. 120.7 (2018), p. 071801. DOI: $10.1103 /$ PhysRevLett.120.071801. arXiv: 1707.07081 [hep-ex] .

[97] M. Tanabashi et al. "Review of Particle Physics". In: Phys. Rev. D 98 (3 Aug. 2018), p. 030001. DOI: 10.1103/PhysRevD .98.030001. URL: https : / / link . aps.org/doi/10.1103/PhysRevD.98.030001.

[98] M. Honda et al. "Improvement of low energy atmospheric neutrino flux calculation using the JAM nuclear interaction model". In: Phys. Rev. D 83 (12 June 2011), p. 123001. DOI: 10.1103 / PhysRevD .83.123001. URL: https: / / link . aps .org/ doi/10.1103/PhysRevD.83.123001.

[99] M. Honda et al. "Atmospheric neutrino flux calculation using the NRLMSISE-00 atmospheric model". In: Physical Review D 92.2 (July 2015). ISSN: 1550-2368. DOI: 10.1103/physrevd.92.023004. URL: http://dx.doi.org/10.1103/ PhysRevD. 92.023004. 
[100] G. Balieiro Gomes et al. "Parameter limits for neutrino oscillation with decoherence in KamLAND”. In: Physical Review D 95.11 (June 2017). ISSN: 2470-0029. DOI: 10 . 1103 / physrevd . 95 .113005. URL: http: / / dx . doi.org/10 .1103/ PhysRevD.95.113005.

[101] João A. B. Coelho, W. Anthony Mann, and Saqib S. Bashar. "Nonmaximal $\theta 23$ Mixing at NOvA from Neutrino Decoherence”. In: Physical Review Letters 118.22 (May 2017). ISSN: 1079-7114. DOI: 10.1103 / physrevlett. 118 . 221801. URL: http : //dx.doi.org/10.1103/PhysRevLett.118.221801.

[102] P. Coloma et al. "Decoherence in neutrino propagation through matter, and bounds from IceCube/DeepCore". In: The European Physical Journal C 78.8 (Aug. 2018). ISSN: 1434-6052. DOI: 10 .1140/epjc/s10052-018-6092-6. URL: http: / / dx . doi.org/10.1140/epjc/s10052-018-6092-6.

[103] G. L. Fogli et al. "Probing nonstandard decoherence effects with solar and KamLAND neutrinos”. In: Physical Review D 76.3 (Aug. 2007). ISSN: 1550-2368. DOI: 10.1103 / physrevd.76.033006. URL: http://dx.doi.org/10.1103/PhysRevD. 76.033006 .

[104] A. M. Gago et al. "Quantum dissipative effects and neutrinos: Current constraints and future perspectives”. In: Physical Review D 63.7 (Feb. 2001). ISSN: 1089-4918. DOI: $10.1103 /$ physrevd.63.073001. URL: http://dx.doi.org/10.1103/ PhysRevD.63.073001.

[105] E. Lisi, A. Marrone, and D. Montanino. "Probing Possible Decoherence Effects in Atmospheric Neutrino Oscillations”. In: Physical Review Letters 85.6 (Aug. 2000), pp. 1166-1169. ISSN: 1079-7114. DOI: 10.1103 /physrevlett.85.1166. URL: http://dx.doi.org/10.1103/PhysRevLett.85.1166.

[106] R. L. N. Oliveira, M. M. Guzzo, and P. C. de Holanda. "Quantum dissipation andCPviolation in MINOS". In: Physical Review D 89.5 (Mar. 2014). ISSN: 1550-2368. DOI: $10.1103 /$ physrevd.89.053002. URL: http://dx.doi .org/10.1103/ PhysRevD.89.053002.

[107] Pouya Bakhti, Yasaman Farzan, and Thomas Schwetz. "Revisiting the quantum decoherence scenario as an explanation for the LSND anomaly". In: Journal of High Energy Physics 2015.5 (May 2015). ISSN: 1029-8479. DOI: 10.1007 / jhep 05 (2015) 007. URL: http: //dx.doi.org/10.1007/JHEP05 (2015) 007. 
[108] Yasaman Farzan, Thomas Schwetz, and Alexei Yu Smirnov. "Reconciling results of LSND, MiniBooNE and other experiments with soft decoherence”. In: Journal of High Energy Physics 2008.7 (2008), pp. 1-20. ISSN: 11266708. DOI: 10 . $1088 / 1126-$ $6708 / 2008 / 07 / 067$. arXiv: 0805.2098.

[109] Roberto L.N. Oliveira. "Dissipative effect in long baseline neutrino experiments". In: European Physical Journal C 76.7 (2016). ISSN: 14346052. DOI: 10.1140 / ep jc / s10052-016-4253-z. arXiv: 1603.08065.

[110] João A. B. Coelho and W. Anthony Mann. "Decoherence, matter effect, and neutrino hierarchy signature in long baseline experiments". In: Physical Review D 96.9 (Nov. 2017). ISSN: 2470-0029. DOI: 10.1103 / physrevd.96.093009. URL: http: //dx.doi.org/10.1103/PhysRevD.96.093009.

[111] A. M. Gago et al. A study on quantum decoherence phenomena with three generations of neutrinos. 2002. arXiv: hep-ph/0208166 [hep-ph].

[112] G. L. Fogli et al. "Status of atmospheric neutrino $\mathrm{v} \mu \rightarrow \mathrm{v} \tau$ oscillations and decoherence after the first K2K spectral data". In: Physical Review D - Particles, Fields, Gravitation and Cosmology 67.9 (2003), pp. 930061-9300614. ISSN: 05562821. DOI: 10 . 1103 / PhysRevD.67.093006. arXiv: 0303064 [hep-ph] .

[113] Luis A. Anchordoqui et al. "Probing Planck scale physics with IceCube". In: Physical Review D - Particles, Fields, Gravitation and Cosmology 72.6 (2005). ISSN: 15507998. DOI: $10.1103 /$ PhysRevD.72.065019. arXiv: 0506168 [hep-ph].

[114] TAKAHIRO SAGAWA. "SECOND LAW-LIKE INEQUALITIES WITH QUANTUM RELATIVE ENTROPY: AN INTRODUCTION”. In: Lectures on Quantum Computing, Thermodynamics and Statistical Physics (Feb. 2012), pp. 125-190. ISSN: 1793-7299. DOI: 10 .1142/9789814425193_0003. URL: http: / / dx.doi .org/ 10 . $1142 / 9789814425193$ _0003. 